\title{
Superdiffusion of Energy in a Chain of Harmonic Oscillators with Noise
}

\author{
Milton Jara ${ }^{1}$, Tomasz Komorowski ${ }^{2}$, Stefano Olla ${ }^{3}$ \\ 1 Institutio Nacional de matematica Pura e Aplicada, Rio de Janeiro, Brazil. \\ E-mail: mjara@impa.br \\ 2 Institute of Mathematics, Polish Academy Of Sciences, Warsaw, Poland. \\ E-mail: komorow@hektor.umcs.lublin.pl \\ 3 CEREMADE, UMR-CNRS 7534, Université Paris Dauphine, Paris, France. \\ E-mail: olla@ceremade.dauphine.fr
}

Received: 1 June 2014 / Accepted: 30 March 2015

Published online: 11 July 2015 - (C) The Author(s) 2015. This article is published with open access at Springerlink.com

\begin{abstract}
We consider a one dimensional infinite chain of harmonic oscillators whose dynamics is perturbed by a stochastic term conserving energy and momentum. We prove that in the unpinned case the macroscopic evolution of the energy converges to the solution of the fractional diffusion equation $\partial_{t} u=-|\Delta|^{3 / 4} u$. For a pinned system we prove that its energy evolves diffusively, generalizing some results of Basile and Olla (J. Stat. Phys. 155(6):1126-1142, 2014).
\end{abstract}

\section{Introduction}

Superdiffusion of energy and the corresponding anomalous thermal conductivity have been observed numerically in the dynamics of unpinned FPU chains $[15,16]$. This is generally attributed to the small scattering rate for low modes, due to momentum conservation. When the interaction has a pinning potential, it is expected that the system undergoes a normal diffusion. More recently, the problem has been studied in models where the Hamiltonian dynamics is perturbed by stochastic terms that conserve energy and momentum, like random exchange of velocity between nearest neighbors particles $[1,2]$. In these models, the interaction is purely harmonic, and as a result, the Green-Kubo formula for thermal conductivity $\kappa$ can be studied explicitly. It diverges for one and two dimensional lattices in case no pinning potential is present, while thermal conductivity stays finite for pinned systems or in dimension $d \geq 3$. In the cases when the conductivity is finite it is proven in [4] that energy fluctuations in equilibrium evolve diffusively.

The main result of the present article concerns the nature of the superdiffusion in dimension 1, when the chain is unpinned. It has already been proven that in the weak noise limit (where the average number of stochastic collisions per unit time is kept finite,

This paper has been partially supported by the European Advanced Grant Macroscopic Laws and Dynamical Systems (MALADY) (ERC AdG 246953), T. K. acknowledges the support of the Polish National Science Center grant UMO-2012/07/B/SR1/03320. 
as in the Grad limit) the Wigner distribution of the energy converges to an inhomogeneous phonon linear Boltzmann equation [5]. Since the corresponding scattering kernel $R\left(k, k^{\prime}\right)$ is positive, the resulting Boltzmann equation can be interpreted probabilistically as the evolution of the density for some Markov process: in this limit a phonon of mode $k$ moves with the velocity given by the gradient of the dispersion relation $\nabla \omega(k)$ and change mode with rate $R\left(k, k^{\prime}\right)$. Under a proper space-time rescaling, this process converges to a Lévy superdiffusion generated by the fractional Laplacian $-|\Delta|^{3 / 4}$. This is proven in $[3,10]$, using probabilistic techniques such as coupling and martingale convergence theorems. A completely analytic proof of the convergence, from a kinetic to a fractional diffusion equation, without the use of the probabilistic representation, has been proposed in [18]. All these results provide a two-step solution: first take a kinetic limit, then use a hydrodynamic rescaling of the kinetic equation. A kind of diagonal procedure is treated in [13]: using the probabilistic approach, one can push the time scale a little longer (matching suitably the size of a still small scattering rate) than in the kinetic limit case. As a result, it is possible to obtain the diffusive limit under the pinning potential and superdiffusive in the unpinned case.

In the present paper we prove a direct limit to the fractional superdiffusion, just by rescaling space and time, without the weak noise assumption. We also recover the diffusive limit results of [4] in the case of a finite diffusivity and study the cases of intermediate weaker noise limits. The rigorous formulations of our main results are listed in Sect. 3.

In a recent article [20], Herbert Spohn predicts the same fractional superdiffusive behavior for the heat mode in the $\beta$-FPU at zero pressure. This follows from an application of mode coupling approximation procedure to fluctuating hydrodynamic equations. Our present results concern a model, which has also three conserved quantities. They are in agreement with the predictions of [20], confirming that the harmonic stochastic model is a good approximation of some non-linear models, at least in the case of symmetric interactions.

The strategy of the proof is as follows: first, we formulate the result for the limit evolution of the Wigner distribution $W_{\epsilon}(t)$ of the energy, when the initial data are in $L^{2}$, see Theorems 5.1 and 5.2, proven in Sects. 10 and 11. These results concern the system with non-equilibrium initial data but of the finite total energy. The extension to homogeneous initial data (whose $L^{2}$ norm is infinite), in particular the equilibrium dynamics with Gibbs distributed initial data, is possible by a simple duality argument, see Sect. 12.

Our results can be formulated in terms of a local energy functional, see Theorems 3.1 and 3.2 in the case of the $L^{2}$ integrable initial data, and Theorem 3.3 for the initial data in equilibrium, respectively. This is possible thanks to the asymptotic equivalence of the relevant energy functionals proven in Propositions 5.3 and 6.3 below.

Concerning the proof when the initial data have square summable realizations, which is the crucial part of our argument, we study first the time evolution of the Wigner distribution of the energy $W_{\epsilon}(t)$, which represents the energy density in both the spatial variable and frequency modes (in fact, it is more convenient to work with the Fourier transform of $W_{\epsilon}(t)$ in the spatial variable). As it has been already remarked in [5], the evolution of $W_{\epsilon}(t)$ is not autonomous but involves another distribution $Y_{\epsilon}(t)$, whose real and imaginary parts represent the difference between kinetic and potential energy and the energy current, respectively, see (9.13). The principal advantage of working with the pair $\left(W_{\epsilon}(t), Y_{\epsilon}(t)\right)$ is that its evolution can be described by a system of ordinary differential equations. By performing the Laplace transform in the temporal domain, the 
system reduces further to an algebraic system of linear equations, see (10.5), and the problem of finding the asymptotics of the energy density for the chain of oscillators reduces to the question of asymptotics of solutions of the system. This is done in Sects. 10 and 11. First, we observe in Sect. 10 that due to the high number of random collisions in the time scales considered, both $W_{\epsilon}(t)$ and $Y_{\epsilon}(t)$ homogenize (unlike in the case of the kinetic limit considered in [5]), and their limits do not depend on the frequency mode variable. The homogenization is proven in Theorem 10.2. In addition, because of fast fluctuations, the time integral of $Y_{\epsilon}(t)$ will disappear from the final equation, as in the case of the kinetic limit in [5]. The above implies that the phonon-Boltzmann equation gives a good approximation of the evolution of $W_{\epsilon}(t)$, but the presence of the error term, which is of order $o(1)$, as $\epsilon$ tends to 0 , does not allow for a direct application of the probabilistic approach of $[3,10]$. Instead, we use a version of the analytic approach of [18], based on projections on the product components of the scattering kernel appearing in the homogenized dynamics, see (5.13) and (10.9) below. This is done in Sect. 11.

In our choice of the dynamics, a diffusive random exchange of momenta takes place between the three nearest neighbor particles in such a way that total kinetic energy and momentum are conserved in the process. However, our method can be applied to linear models with quite general stochastic scattering mechanisms, generating different scattering rates. The result does not depend on the particular type of stochastic perturbation, as long as it conserves the appropriate quantities. E.g., we could consider a model with a simple Poissonian exchange of the two nearest neighbor velocities described in Sect. 2.1.3 below. In fact this case is computationally less involved, due to a simpler structure of the respective scattering kernel.

Concerning the possible generalizations of our results to dimensions $d \geq 2$, see [1] for the formulation of the model; we conjecture they can also be treated by the present method.

Although for the equilibrium fluctuations we prove only the convergence of the covariance function, our approach can be further developed to obtain the convergence in law for the equilibrium fluctuation field to the respective Ornstein-Uhlenbeck process. The question of the convergence in probability for the non-equilibrium case could possibly be more involved, as it requires the control of higher moments of the energy distribution.

A remark concerning the initial data of the system is also in order. We choose the initial probability distributions of the velocities and inter-particle distances whose energy spectrum satisfies condition (3.9). This condition implies that the initial data are macroscopically centered (see Sect. 3.2.1). While this choice is quite natural in the situation of a pinned chain, it requires some explanation in the unpinned case. In the latter situation, if we start with non-centered initial conditions, their respective macroscopic averages will evolve, at the hyperbolic space-time scale, following the linear wave equation. As a result, they will disperse to infinity, since we start with the data whose realization has a finite $L^{2}$ norm. This implies that at a larger superdiffusive time scale these averages will be null. Thus the initial condition for the macroscopic superdiffusive evolution is provided solely by the variance or the high oscillations of the initial data, i.e., the temperature profile. Summarizing, the initial energy profile can be decomposed into a temperature profile and a phonon energy profile. At the hyperbolic time scale the phonon energy profile will converge to 0 asymptotically, as time goes to infinity, while the temperature profile remains stationary. The latter starts evolving at the larger, superdiffusive time scale following a fractional heat equation. The details of this decomposition will be explained in a forthcoming article [11]. 
We mention here the article [6], where a result similar to ours is proven, by very different techniques, for a dynamics with two conserved quantities (energy and volume) in the case when the initial data is given by a Gibbs equilibrium measure.

\section{The Dynamics}

\subsection{Infinite chain of interacting harmonic oscillators.}

2.1.1. Hamiltonian system. The dynamics of the chain of oscillators can be written formally as a Hamiltonian system of differential equations

$$
\begin{aligned}
& \dot{\mathfrak{q}}_{x}(t)=\partial_{\mathfrak{p}_{x}} \mathcal{H}(\mathfrak{p}(t), \mathfrak{q}(t)) \\
& \dot{\mathfrak{p}}_{x}(t)=-\partial_{\mathfrak{q}_{x}} \mathcal{H}(\mathfrak{p}(t), \mathfrak{q}(t)), \quad x \in \mathbb{Z} .
\end{aligned}
$$

The formal Hamiltonian is given by

$$
\mathcal{H}(\mathfrak{p}, \mathfrak{q}):=\frac{1}{2} \sum_{x \in \mathbb{Z}} \mathfrak{p}_{x}^{2}+\frac{1}{2} \sum_{x, x^{\prime} \in \mathbb{Z}} \alpha_{x-x^{\prime}} \mathfrak{q}_{x} \mathfrak{q}_{x^{\prime}}
$$

and we assume also (cf [5]) that:

(a1) $\left(\alpha_{x}\right)_{x \in \mathbb{Z}}$ is real valued and there exists $C>0$ such that $\left|\alpha_{x}\right| \leq C e^{-|x| / C}$ for all $x \in \mathbb{Z}$,

(a2) $\hat{\alpha}(k)$ is also real valued and $\hat{\alpha}(k)>0$ for $k \neq 0$ and in case $\hat{\alpha}(0)=0$ we have $\hat{\alpha}^{\prime \prime}(0)>0$,

(a3) to guarantee that the local energy functional, see (3.8) below, is non-negative we assume that $\alpha_{x} \leq 0, x \neq 0$.

Here $\hat{\alpha}(k)$ is the Fourier transform of sequence $\left(\alpha_{x}\right)_{x}$, defined as

$$
\hat{\alpha}(k)=\sum_{x} \alpha_{x} \exp \{-2 \pi i x k\}, \quad k \in \mathbb{T} .
$$

The above conditions imply that both functions $x \mapsto \alpha_{x}$ and $k \mapsto \hat{\alpha}(k)$ are even. In addition, $\hat{\alpha} \in C^{\infty}(\mathbb{T})$. Define the dispersion relation as $\omega(k):=\hat{\alpha}^{1 / 2}(k)$. In case when $\hat{\alpha}(0)>0$ the dispersion relation belongs to $C^{\infty}(\mathbb{T})$. If $\hat{\alpha}(0)=0$ we can write

$$
\omega(k)=|\sin (\pi k)| \sqrt{\frac{\hat{\alpha}^{\prime \prime}(0)}{2 \pi^{2}}} \varphi\left(\sin ^{2}(\pi k)\right),
$$

where $\varphi:[0,+\infty) \rightarrow(0,+\infty)$ is of $C^{2}$ class and such that $\varphi(0)=1$.

The $\mathfrak{p}_{x}$ component stands for the velocity (or momentum, as the mass of each particle is taken equal to 1 ) of the particle $x$. In the pinned case, $\hat{\alpha}(0)>0$, the particle labelled with $x$ feels a pinning harmonic potential centered at $a x$, where $a \geq 0$ is an arbitrary equilibrium interparticle distance, so $\mathfrak{q}_{x}$ should be interpreted as the displacement of the position of the particle $x$ from the point $a x$. Since the dynamics is linear, it does not depend on $a$, which assume is equal to 1 .

In the unpinned case, $\hat{\alpha}(0)=0$, the system is translation invariant, and only the interparticle distances are relevant for the dynamics. So in the unpinned case the variables $\mathfrak{q}_{x}$ are defined up to a common additive constant. Therefore, the relevant quantities are functionals of the relative distances between the particles. An important example is a 
wave function defined in Sect. 5.1. Its definition is unambiguous both in the pinned and unpinned cases. In the unpinned case, the total momentum and the energy of the chain are formally conserved, (besides the volume of course). Since we insist on preserving these properties, we choose a stochastic perturbation having the same conservation laws. This can be done either locally, via a time continuous stochastic exchange of momentum considered in this paper, or through a time discontinuous random exchange of momentum mechanism (see Sect. 2.1.3).

2.1.2. Continuous time noise. We add to the right hand side of (2.1) a local stochastic term that conserves $\mathfrak{p}_{x-1}^{2}+\mathfrak{p}_{x}^{2}+\mathfrak{p}_{x+1}^{2}$ and $\mathfrak{p}_{x-1}+\mathfrak{p}_{x}+\mathfrak{p}_{x+1}$. The respective stochastic differential equations can be written as

$$
\begin{aligned}
d \mathfrak{q}_{x}(t)= & \mathfrak{p}_{x}(t) d t \\
d \mathfrak{p}_{x}(t)= & {\left[-(\alpha * \mathfrak{q}(t))_{x}-\frac{\gamma}{2}(\beta * \mathfrak{p}(t))_{x}\right] d t } \\
& +\gamma^{1 / 2} \sum_{z=-1,0,1}\left(Y_{x+z} \mathfrak{p}_{x}(t)\right) d w_{x+z}(t), \quad x \in \mathbb{Z},
\end{aligned}
$$

with the parameter $\gamma>0$ that determines the strength of the noise in the system, and $\left(Y_{x}\right)$ are vector fields given by

$$
Y_{x}:=\left(\mathfrak{p}_{x}-\mathfrak{p}_{x+1}\right) \partial_{\mathfrak{p}_{x-1}}+\left(\mathfrak{p}_{x+1}-\mathfrak{p}_{x-1}\right) \partial_{\mathfrak{p}_{x}}+\left(\mathfrak{p}_{x-1}-\mathfrak{p}_{x}\right) \partial_{\mathfrak{p}_{x+1}}
$$

Here $\left(w_{x}(t)\right)_{t \geq 0}, x \in \mathbb{Z}$ are i.i.d. one dimensional, real valued, standard Brownian motions, that are non-anticipative over some filtered probability space $\left(\Omega, \mathcal{F},\left(\mathcal{F}_{t}\right), \mathbb{P}\right)$. Furthermore, $\beta_{x}=\Delta \beta_{x}^{(0)}$, where

$$
\beta_{x}^{(0)}=\left\{\begin{aligned}
-4, & x=0, \\
-1, & x= \pm 1, \\
0, & \text { if otherwise. }
\end{aligned}\right.
$$

The lattice Laplacian of $\left(g_{x}\right)_{x \in \mathbb{Z}}$ is defined as $\Delta g_{x}:=g_{x+1}+g_{x-1}-2 g_{x}$. Let also $\nabla g_{x}:=g_{x+1}-g_{x}$ and $\nabla^{*} g_{x}:=g_{x-1}-g_{x}$. For a future reference we let $\beta_{1, x}:=\nabla^{*} \beta_{x}^{(0)}$. A simple calculation shows that

$$
\hat{\beta}(k)=8 \mathfrak{s}^{2}(k)\left[1+2 \mathfrak{c}^{2}(k)\right]=8 \mathfrak{s}^{2}(k)+4 \mathfrak{s}^{2}(2 k)
$$

and

$$
\hat{\beta}_{1}(k)=\left(1-e^{-2 i \pi k}\right)\left(4+e^{2 \pi i k}+e^{-2 \pi i k}\right),
$$

where, for the abbreviation sake, we have written

$$
\mathfrak{s}(k):=\sin (\pi k) \quad \text { and } \quad \mathfrak{c}(k):=\cos (\pi k), \quad k \in \mathbb{T} .
$$

2.1.3. Random momentum exchange. Another possible stochastic dynamics that conserves the volume, energy and momentum (in the unpinned case) can be obtained by a "jump" type mechanism of the momentum exchange. More precisely, let $\left(N_{x, x+1}(t)\right)_{x \in \mathbb{Z}}$ be i.i.d. Poisson processes with intensity $3 \gamma / 2$. The dynamics of the position component $\left(\mathfrak{q}_{x}(t)\right)_{x \in \mathbb{Z}}$ is the same as in (2.5), while the momentum $\left(\mathfrak{p}_{x}(t)\right)_{x \in \mathbb{Z}}$ is a càdlàg process given by

$$
\begin{aligned}
d \mathfrak{p}_{x}(t)= & -(\alpha * \mathfrak{q}(t))_{x} d t \\
& +\left[\nabla \mathfrak{p}_{x}(t-) d N_{x, x+1}(t)+\nabla^{*} \mathfrak{p}_{x}(t-) d N_{x-1, x}(t)\right], \quad x \in \mathbb{Z} .
\end{aligned}
$$




\section{Main Results: Macroscopic Evolution}

3.1. Remarks on hyperbolic scaling. Euler equations. Consider now the unpinned case $\hat{\alpha}(0)=0$. For a configuration $\left(\mathfrak{p}_{x}(t), \mathfrak{r}_{x}(t)\right)_{x \in \mathbb{Z}}$ we define the energy per atom:

$$
\mathfrak{e}_{x}(t)=\frac{\mathfrak{p}_{x}^{2}(t)}{2}-\frac{1}{4} \sum_{y} \alpha_{x-y}\left(\mathfrak{q}_{x}(t)-\mathfrak{q}_{y}(t)\right)^{2} .
$$

Thanks to condition (a3) we have $\mathfrak{e}_{x}(t) \geq 0$. Notice also that, since $\sum_{x} \alpha_{x}=0$, formally we have $\sum_{x} \mathfrak{e}_{x}(t)=\mathcal{H}(\mathfrak{p}(t), \mathfrak{q}(t))$.

Define $\mathfrak{r}_{x}(t)=\mathfrak{q}_{x}(t)-\mathfrak{q}_{x-1}(t)$. Then $\sum_{x} \mathfrak{r}_{x}(t)$, when finite, represents the total length of the system when the equilibrium interparticle distance $a=0$. The chain has three formally conserved (also called balanced) quantities

$$
\begin{aligned}
& \sum_{x} \mathfrak{r}_{x}(t) \text {-volume (length), } \\
& \sum_{x} \mathfrak{p}_{x}(t) \text { - momentum, } \\
& \sum_{x} \mathfrak{e}_{x}(t) \text { - energy. }
\end{aligned}
$$

Because the noise is added to the system, these are the 'only' conserved quantities. More precisely, the only stationary probability measures for the infinite dynamics (2.1), that are also translation invariant and have a finite density entropy property (see Definition 4.2.1 of [7]), are mixtures of the Gibbs measures

$$
d \mu_{T, \bar{p}, \tau}=\frac{1}{Z_{T, \bar{p}, \tau}} \exp \left\{-T^{-1}\left(\mathcal{H}-\bar{p} \sum_{x} \mathfrak{p}_{x}-\tau \sum_{x} \mathfrak{r}_{x}\right)\right\} \prod_{x} d \mathfrak{r}_{x} d \mathfrak{p}_{x}
$$

parametrized by the temperature $T$, momentum $\bar{p}$ and tension $\tau$, properly defined locally by the appropriate DLR equations on their conditional distributions (see Section 4 of [7]).

It can be proven that after the hyperbolic space-time scaling, these conserved quantities evolve deterministically following the system of Euler equations:

$$
\left\{\begin{aligned}
\partial_{t} \bar{r}(t, y) & =\partial_{y} \bar{p}(t, y), \\
\partial_{t} \bar{p}(t, y) & =\tau_{1} \partial_{y} \bar{r}(t, y), \\
\partial_{t} \bar{e}(t, y) & =\tau_{1} \partial_{y}(\bar{r}(t, y) \bar{p}(t, y)),
\end{aligned}\right.
$$

with the initial data

$$
\bar{r}(0, y)=\bar{r}_{0}(y), \quad \bar{p}(0, y)=\bar{p}_{0}(y), \quad \bar{e}(0, y)=\bar{e}_{0}(y)
$$

determined by the limits of quantities given by (3.2) at time $t=0$. Here the parameter $\tau_{1}$, called the sound speed, is defined by

$$
\tau_{1}:=\frac{\hat{\alpha}^{\prime \prime}(0)}{8 \pi^{2}} .
$$

More precisely, consider the empirical distributions associated to the conserved quantities: $\mathfrak{u}_{x}(t):=\left(\mathfrak{r}_{x}(t), \mathfrak{p}_{x}(t), \mathfrak{e}_{x}(t)\right)$. Then, 


$$
\lim _{\epsilon \rightarrow 0+} \epsilon \sum_{x} J(\epsilon x) \mathfrak{u}_{x}\left(\epsilon^{-1} t\right)=\int_{\mathbb{R}} J(y) \bar{u}(t, y) d y,
$$

with $J$-a smooth test function with compact support, and the convergence holds in probability for any $t>0$, provided it holds for the initial distribution at $t=0$. The functions $\bar{r}_{0}, \bar{p}_{0}, \bar{e}_{0}$ are assumed to belong to $C_{0}^{\infty}(\mathbb{R})$ - the space of all smooth and compactly supported functions. The components of $\bar{u}(t, y):=(\bar{r}(t, y), \bar{p}(t, y), \bar{e}(t, y))$ satisfy (3.3). Note that system (3.3) decouples. Quantities $(\bar{r}(t, y), \bar{p}(t, y))$ satisfy the linear wave equation. Define the energy of the phonon modes as

$$
\bar{e}_{\mathrm{ph}}(t, y):=\frac{\tau_{1} \bar{r}^{2}(t, y)}{2}+\frac{\bar{p}^{2}(t, y)}{2} .
$$

The residual energy component, called the local temperature profile, is given by

$$
T(y):=\bar{e}_{0}(y)-\bar{e}_{\mathrm{ph}}(0, y) .
$$

The above definition leads to the decomposition of the energy profile $\bar{e}(t, y)$ into the temperature profile, that remains stationary under the hyperbolic scaling, and the phononic energy $\bar{e}_{\mathrm{ph}}(t, y)$ whose evolution is driven by the linear wave equation, see (3.3). Observe that, starting with compactly supported initial data, the phonon energy will disperse to infinity, as $t \rightarrow \infty$, and the energy profile will converge (weakly) to the temperature profile. This is the reason why at any larger time scale, we have only to look at the evolution of the temperature profile.

In the case of a finite number of particles $N=\left[\epsilon^{-1}\right]$, with periodic or other boundary conditions, convergence in probability stated in (3.5) can be proven by using relative entropy methods, see [19] and [8]. In fact in the latter paper the limit has been shown in the non-linear case, in the smooth regime of the Euler equations. In the infinite volume, starting with the initial distribution $\mu_{\epsilon}$ on the space of configurations $\left(\mathfrak{r}_{x}, \mathfrak{p}_{x}\right)_{x \in \mathbb{Z}}$ satisfying

$$
\sup _{\epsilon \in(0,1]} \epsilon\langle\mathcal{H}(\mathfrak{p}, \mathfrak{q})\rangle_{\mu_{\epsilon}}<+\infty
$$

with $\langle\cdot\rangle_{\mu_{\epsilon}}$ denoting the expectation with respect to $\mu_{\epsilon}$, the relative entropy method cannot be applied.

The detailed analysis of the behavior of the energy component corresponding to the phononic modes, under the hyperbolic scaling is not the subject of the present paper and we shall deal with it in our future work. Our purpose here is to go beyond the hyperbolic time scale and understand the behavior of the energy component corresponding to the local temperature profile on the diffusive or (if necessary) superdiffusive space-time scale.

3.2. Behavior of the energy functional. Our main results deal with the macroscopic behavior of the energy functional, for a given configuration $(\mathfrak{p}(t), \mathfrak{q}(t))$. The energy per site is defined as

$$
\mathfrak{e}_{x}(t):=\frac{\mathfrak{p}_{x}^{2}(t)}{2}-\frac{1}{4} \sum_{y} \alpha_{x-y}\left(\mathfrak{q}_{x}(t)-\mathfrak{q}_{y}(t)\right)^{2}+\frac{\hat{\alpha}(0)}{2} \mathfrak{q}_{x}^{2}(t) .
$$

In this section we shall assume that condition (3.7) is satisfied. Denote by $\mathbb{E}_{\epsilon}$ the expectation with respect to the product measure $\mathbb{P}_{\epsilon}:=\mu_{\epsilon} \otimes \mathbb{P}$. 
3.2.1. Superdiffusive behavior of the unpinned chain. We assume first that $\hat{\alpha}(0)=0$, i.e. the pinning potential vanishes and the Hamiltonian dynamics conserves both the momentum and energy.

Define, the energy spectrum of a configuration $\left(\mathfrak{p}_{x}, \mathfrak{q}_{x}\right)_{x \in \mathbb{Z}}$ as

$$
\mathfrak{w}_{\epsilon}(k):=\left\langle|\hat{\mathfrak{p}}(k)|^{2}+\hat{\alpha}(k)|\hat{\mathfrak{q}}(k)|^{2}\right\rangle_{\mu_{\epsilon}}, \quad k \in \mathbb{T},
$$

where $\hat{\mathfrak{p}}(k)$ and $\hat{\mathfrak{q}}(k)$ are the Fourier transforms of $\left(\mathfrak{p}_{x}\right)$ and $\left(\mathfrak{q}_{x}\right)$, respectively (see Sect. 4 below), and $\hat{\alpha}(k)$ is given by (2.3). Assumption (3.7) is equivalent with

$$
\sup _{\epsilon \in(0,1]} \epsilon \int_{\mathbb{T}} \mathfrak{w}_{\epsilon}(k) d k<+\infty .
$$

In what follows we shall suppose a stronger integrability condition on $\mathfrak{w}_{\epsilon}(k)$. Namely, we assume that

$$
\sup _{\epsilon \in(0,1]} \epsilon^{2} \int_{\mathbb{T}} \mathfrak{w}_{\epsilon}^{2}(k) d k<+\infty
$$

According to the remark made below formula (4.9) the above assumption implies that both

$$
\lim _{\epsilon \rightarrow 0+} \epsilon \sum_{x} J(\epsilon x)\left\langle\mathfrak{r}_{x}\right\rangle_{\mu_{\epsilon}}=0
$$

and

$$
\lim _{\epsilon \rightarrow 0+} \epsilon \sum_{x} J(\epsilon x)\left\langle\mathfrak{p}_{x}\right\rangle_{\mu_{\epsilon}}=0, \quad \forall J \in C_{0}^{\infty}(\mathbb{R}) .
$$

Suppose that the initial distribution of energy satisfies the following assumptions:

$$
\lim _{\epsilon \rightarrow 0+} \epsilon \sum_{x} J(\epsilon x)\left\langle\mathfrak{e}_{x}\right\rangle_{\mu_{\epsilon}}=\int_{\mathbb{R}} J(y) W_{0}(y) d y,
$$

where $W_{0} \in L^{1}(\mathbb{R})$ (it is obviously non-negative).

Theorem 3.1. Let $\delta=3 / 2$, then, under the conditions on the initial distribution stated in the foregoing, for any test function $J \in C_{0}^{\infty}([0,+\infty) \times \mathbb{R})$ we have:

$$
\lim _{\epsilon \rightarrow 0+} \epsilon \sum_{x} \int_{0}^{+\infty} J(t, \epsilon x) \mathbb{E}_{\epsilon} \mathfrak{e}_{x}\left(\frac{t}{\epsilon^{\delta}}\right) d t=\int_{0}^{+\infty} \int_{\mathbb{R}} W(t, y) J(t, y) d t d y,
$$

where $W(t, y)$ satisfies the fractional heat equation:

$$
\partial_{t} W(t, y)=-\hat{c}\left|\Delta_{y}\right|^{3 / 4} W(t, y)
$$

with the initial condition $W(0, y)=W_{0}(y)$ and

$$
\hat{c}:=\frac{\left[\alpha^{\prime \prime}(0)\right]^{3 / 4}}{2^{9 / 4}(3 \gamma)^{1 / 2}} \text {. }
$$

The proof of this result is a direct consequence of Theorem 5.2 and Proposition 5.3 formulated below. In fact, (as can be seen from the aforementioned results) it can be formulated in a more general way to cover also the case of a weaker noise, i.e. parameter $\gamma$ can be replaced by $\epsilon^{s} \gamma_{0}$, for some $s \in[0,1)$ and $\gamma_{0}>0$. Then, the result is still valid at the time scale corresponding to the exponent $\delta=(3-s) / 2$. The limit $W(t, y)$ is the same as in the case $s=0$, covered by Theorem 3.1. 
3.2.2. Diffusive behavior of the pinned chain. If $\hat{\alpha}(0)>0$ there is a pinning potential and the Hamiltonian dynamics does not conserve the momentum. Energy is the only relevant conserved quantity but it does not evolve at the hyperbolic space-time scale. Define

$$
\hat{\sigma}^{2}:=\int_{\mathbb{T}} \frac{\left[\omega^{\prime}(k)\right]^{2}}{R(k)} d k
$$

where

$$
R(k):=\frac{\hat{\beta}(k)}{4} .
$$

Since $\omega^{\prime}(k) \approx k$ and $R(k) \approx k^{2}$, as $k \ll 1$ (see (2.4) and (2.7)), we have $\hat{\sigma}^{2}<+\infty$ (it is infinite in the unpinned case, due to $\omega^{\prime}(k) \approx \operatorname{sign} k$ ). As a result, the evolution is diffusive and we have the following:

Theorem 3.2. Let $s \in[0,1), \gamma=\epsilon^{s} \gamma_{0}$. Then, under the assumptions made in the foregoing, for any $J(t, y)$ as in Theorem 3.1 we have

$$
\lim _{\epsilon \rightarrow 0+} \epsilon \int_{0}^{+\infty}\left[\sum_{x} J(t, \epsilon x) \mathbb{E}_{\epsilon} \mathfrak{e}_{x}\left(\frac{t}{\epsilon^{\delta}}\right)\right] d t=\int_{0}^{+\infty} \int_{\mathbb{R}} W(t, y) J(t, y) d t d y,
$$

with $\delta=2-s$, where $W(t, y)$ satisfies the heat equation:

$$
\partial_{t} W(t, y)=\hat{c} \partial_{y}^{2} W(t, y) .
$$

Here

$$
\hat{c}=\frac{\hat{\sigma}^{2}}{\gamma_{0}}+8 \gamma_{0} \pi^{2} \quad \text { if } s=0,
$$

and

$$
\hat{c}=\frac{\hat{\sigma}^{2}}{\gamma_{0}} \quad \text { if } 0<s<1 .
$$

The above theorem follows directly from Theorem 5.1 and the already mentioned Proposition 5.3 formulated below.

3.3. Equilibrium fluctuations. The results formulated in Sect. 3.2 hold under the condition of finite microscopic total energy (3.7). By a duality argument they can be applied to obtain the following macroscopic behavior of the fluctuations when the system starts in an equilibrium measure $\mu_{\mathcal{E}_{0}, 0,0}$. For the fluctuations of the energy mode we assume that $\gamma=\gamma_{0} \epsilon^{s}$ for some $\gamma_{0}>0$ and $s \in[0,1)$. Consider the energy fluctuation field

$$
\tilde{\mathfrak{e}}_{\epsilon}(t, J)=\sqrt{\epsilon} \sum_{x} J(\epsilon x)\left[\mathfrak{e}_{x}\left(\frac{t}{\epsilon^{\delta}}\right)-\mathcal{E}_{0}\right], \quad J \in C_{0}^{\infty}(\mathbb{R}),
$$

where $\mathfrak{e}_{x}(t)$ is given by (3.8) and $\delta$ is chosen as before, i.e. $\delta=(3-s) / 2$ in the unpinned case, and $\delta=2-s$ in the pinned one. The covariance field is defined as

$$
C_{\epsilon}^{(e)}\left(t, J_{1}, J_{2}\right):=\mathbb{E}\left[\tilde{\mathfrak{e}}_{\epsilon}\left(t, J_{1}\right) \tilde{\mathfrak{e}}_{\epsilon}\left(0, J_{2}\right)\right], \quad J_{1}, J_{2} \in C_{0}^{\infty}(\mathbb{R}) .
$$

The following theorem is a direct corollary from Theorems 6.1 and 6.2, and Proposition 6.3 formulated below. 
Theorem 3.3. For any functions $J_{1}, J_{2} \in C_{0}^{\infty}(\mathbb{R})$ and $\phi \in L^{1}[0,+\infty)$ we have

$$
\lim _{\epsilon \rightarrow 0} \int_{0}^{+\infty} \phi(t) C_{\epsilon}^{(e)}\left(t, J_{1}, J_{2}\right) d t=\int_{0}^{+\infty} \phi(t) C^{(e)}\left(t, J_{1}, J_{2}\right) d t
$$

where $C^{(e)}\left(t, J_{1}, J_{2}\right)$ satisfies the equation

$$
\partial_{t} C^{(e)}\left(t, J_{1}, J_{2}\right)=C^{(e)}\left(t, \mathcal{A} J_{1}, J_{2}\right)
$$

with the initial condition

$$
C^{(e)}\left(0, J_{1}, J_{2}\right)=\mathcal{E}_{0} \int_{\mathbb{R}} J_{1}(y) J_{2}(y) d y,
$$

and $\mathcal{A}=-\hat{c}\left|\Delta_{y}\right|^{3 / 4}$ in the unpinned, or $\mathcal{A}=D \Delta_{y}$ in the pinned case, respectively. Coefficients $\hat{c}$ and $D$ are the same as in Theorems 3.1 and 3.2, respectively.

Remark. We remark here that Theorems 3.1 through 3.3 hold also for the dynamics corresponding to the random momentum exchange model described by (2.10).

\section{Some Basic Notation}

The one dimensional torus $\mathbb{T}$ is the interval $[-1 / 2,1 / 2]$ with identified endpoints. Let $\ell^{2}$ be the space of all complex valued sequences $\left(\psi_{x}\right)_{x \in \mathbb{Z}}$, equipped with the norm $\|\psi\|_{\ell^{2}}^{2}:=\sum_{x}\left|\psi_{x}\right|^{2}$. For $m \in \mathbb{R}$ we introduce $h_{m}$-the space of sequences $\left(\psi_{x}\right)_{x \in \mathbb{Z}}$, for which $\|\psi\|_{h_{m}}^{2}:=\sum_{x}\left(1+x^{2}\right)^{m}\left|\psi_{x}\right|^{2}<+\infty$.

Given a sequence $\left(\psi_{x}\right) \in \ell^{2}$ define $\hat{\psi}: \mathbb{T} \rightarrow \mathbb{C}$-its Fourier transform-by (2.3). Obviously $\hat{\psi}$ belongs to $L^{2}(\mathbb{T})$ - the space of all complex valued functions equipped with the norm $\|\hat{\psi}\|_{L^{2}(\mathbb{T})}:=\langle\hat{\psi}, \hat{\psi}\rangle_{L^{2}(\mathbb{T})}^{1 / 2}$, where

$$
\langle\hat{\psi}, \hat{\phi}\rangle_{L^{2}(\mathbb{T})}:=\int_{\mathbb{T}} \hat{\psi}(k) \hat{\phi}^{*}(k) d k
$$

Formula (2.3) determines also an isometric isomorphism between $h_{m}$ and $H^{m}(\mathbb{T})$ - the completion of $C^{\infty}(\mathbb{T})$ in the norm $\|\hat{\psi}\|_{H^{m}(\mathbb{T})}:=\|\psi\|_{h_{m}}$. We have $H^{0}(\mathbb{T})=L^{2}(\mathbb{T})$.

For an arbitrary $J: \mathbb{T} \rightarrow \mathbb{C}, k \in \mathbb{T}, p \in \mathbb{R}$ and $\epsilon>0$ we define

$$
\begin{aligned}
\delta_{\epsilon} J(p, k) & :=\frac{1}{\epsilon}\left[J\left(k+\frac{\epsilon p}{2}\right)-J\left(k-\frac{\epsilon p}{2}\right)\right], \\
\bar{J}(k, p) & :=\frac{1}{2}\left[J\left(k+\frac{p}{2}\right)+J\left(k-\frac{p}{2}\right)\right] .
\end{aligned}
$$

Given a set $A$ and two functions $f, g: A \rightarrow \mathbb{R}_{+}$we say that $f(x) \approx g(x), x \in A$ if there exists $C>1$ such that

$$
\frac{f(x)}{C} \leq g(x) \leq C f(x), \quad \forall x \in A .
$$

We write $g(x) \preceq f(x)$, when only the upper bound on $g$ is satisfied.

Denote by $\mathcal{S}$ the set of functions $J: \mathbb{R} \times \mathbb{T} \rightarrow \mathbb{C}$ that are of $C^{\infty}$ class and such that for any integers $l, m, n$ we have 


$$
\sup _{y \in \mathbb{R}, k \in \mathbb{T}}\left(1+y^{2}\right)^{n}\left|\partial_{y}^{l} \partial_{k}^{m} J(y, k)\right|<+\infty .
$$

For $J \in \mathcal{S}$ we let $\hat{J}$ be its Fourier transform in the first variable, i.e.

$$
\hat{J}(p, k):=\int_{\mathbb{R}} e^{-2 \pi i y p} J(y, k) d y .
$$

We introduce the norm

$$
\|J\|_{\mathcal{A}}:=\int_{\mathbb{R}} \sup _{k}|\hat{J}(p, k)| d p .
$$

By $\mathcal{A}$ we denote the completions of $\mathcal{S}$ in the respective norm.

4.1. Averaged Wigner transform. For a given $\epsilon \in(0,1]$ we let $\psi$ be a random element distributed on $\ell^{2}$ according to a Borel probability measure $\mu_{\epsilon}$. We assume that ( $\left.\mathrm{cf}(3.9)\right)$

$$
K_{1}:=\sup _{\epsilon \in(0,1]} \int_{\mathbb{T}} d k\left[\epsilon\left\langle|\hat{\psi}(k)|^{2}\right\rangle_{\mu_{\epsilon}}\right]^{2}<+\infty
$$

where $\langle\cdot\rangle_{\mu_{\epsilon}}$ is the expectation with respect to $\mu_{\epsilon}$.

Define $W_{\epsilon}^{(0)}, Y_{\epsilon}^{(0)} \in \mathcal{A}^{\prime}$

$$
\left\langle W_{\epsilon}^{(0)}, J\right\rangle:=\frac{\epsilon}{2} \int_{\mathbb{R} \times \mathbb{T}}\left\langle\hat{\psi}^{*}\left(k-\frac{\epsilon p}{2}\right) \hat{\psi}\left(k+\frac{\epsilon p}{2}\right)\right\rangle_{\mu_{\epsilon}} \hat{J}^{*}(p, k) d p d k,
$$

and

$$
\left\langle Y_{\epsilon}^{(0)}, J\right\rangle:=\frac{\epsilon}{2} \int_{\mathbb{R} \times \mathbb{T}}\left\langle\hat{\psi}\left(k+\frac{\epsilon p}{2}\right) \hat{\psi}\left(-k+\frac{\epsilon p}{2}\right)\right\rangle_{\mu_{\epsilon}} \hat{J}^{*}(p, k) d p d k
$$

for any $J \in \mathcal{A}$. From the Cauchy-Schwartz inequality we get

$$
\left|\left\langle W_{\epsilon}^{(0)}, J\right\rangle\right| \leq \frac{\epsilon}{2}\|J\|_{\mathcal{A}}\left\langle\|\hat{\psi}\|_{L^{2}(\mathbb{T})}^{2}\right\rangle_{\mu_{\epsilon}} .
$$

Thanks to Jensen's inequality we conclude from (4.3) that

$$
K_{0}:=\sup _{\epsilon \in(0,1]} \frac{\epsilon}{2}\left\langle\|\hat{\psi}\|_{L^{2}(\mathbb{T})}^{2}\right\rangle_{\mu_{\epsilon}}<+\infty
$$

Therefore

$$
\sup _{\epsilon \in(0,1]}\left(\left\|Y_{\epsilon}^{(0)}\right\|_{\mathcal{A}^{\prime}}+\left\|W_{\epsilon}^{(0)}\right\|_{\mathcal{A}^{\prime}}\right) \leq 2 K_{0}
$$

Functional $W_{\epsilon}^{(0)} \in \mathcal{A}^{\prime}$ is called the averaged Wigner transform of $\psi$. We refer to $Y_{\epsilon}^{(0)}$ as the averaged anti-Wigner transform. By Plancherel's identity we obtain

$$
\left\langle W_{\epsilon}^{(0)}, J\right\rangle=\frac{\epsilon}{2} \sum_{x, x^{\prime} \in \mathbb{Z}}\left\langle\left(\psi_{x^{\prime}}\right)^{*} \psi_{x}\right\rangle_{\epsilon} \int_{\mathbb{T}} e^{2 \pi i\left(x^{\prime}-x\right) k} J^{*}\left(\frac{\epsilon}{2}\left(x+x^{\prime}\right), k\right) d k,
$$

for any $J \in \mathcal{S}$. As a consequence of (4.7), both $\left(W_{\epsilon}^{(0)}\right)_{\epsilon}$ and $\left(Y_{\epsilon}^{(0)}\right)_{\epsilon}$ are $*$-weakly (sequentially) compact in $\mathcal{A}^{\prime}$, as $\epsilon \rightarrow 0+$, i.e. for any sequence $\epsilon_{n} \rightarrow 0$ we can choose a 
subsequence $\left(W_{\epsilon_{n}^{\prime}}^{(0)}, Y_{\epsilon_{n}^{\prime}}^{(0)}\right)_{n \geq 1}$ whose each component is $*$-weakly convergent in $\mathcal{A}^{\prime}$, see Section 4.1 of [5].

One can show, see Theorem B4 of [17], that if $\left(W_{\epsilon_{n}^{\prime}}^{(0)}\right)_{n}$ is $*$-weakly convergent then there exists a finite Borel measure $W_{0}(d y, d k)$ on $\mathbb{R} \times \mathbb{T}$ whose total mass does not exceed $K_{0}$ and such that

$$
\lim _{n \rightarrow+\infty}\left\langle W_{\epsilon_{n}}^{(0)}, J\right\rangle=\int_{\mathbb{R} \times \mathbb{T}} J^{*}(y, k) W_{0}(d y, d k), \quad J \in \mathcal{A} .
$$

Applied to functions $J(y, k)=J(y)$ the Wigner distribution becomes:

$$
\left\langle W_{\epsilon}^{(0)}, J\right\rangle=\frac{\epsilon}{2} \sum_{x}\left\langle\left|\psi_{x}\right|^{2}\right\rangle_{\mu_{\epsilon}} J(\epsilon x) .
$$

Remark. Observe that condition (4.3) implies that $\left(\psi_{x}\right)_{x}$ is centered in the following sense: for any $J \in C_{0}^{\infty}(\mathbb{R})$ we have

$$
\lim _{\epsilon \rightarrow 0+} \epsilon \sum_{x}\left\langle\psi_{x}\right\rangle_{\mu_{\epsilon}} J(\epsilon x)=0 .
$$

Indeed, by Plancherel's identity we can write that the absolute value of the expression under the limit equals

$$
\epsilon\left|\int_{\mathbb{T}}\langle\hat{\psi}(k)\rangle_{\mu_{\epsilon}} \hat{J}_{\epsilon}(k) d k\right|
$$

where

$$
\hat{J}_{\epsilon}(k):=\sum_{x} J(\epsilon x) \exp \{-2 \pi i k x\} \approx \frac{1}{\epsilon} \hat{J}\left(\frac{k}{\epsilon}\right)
$$

and $\hat{J}(k)$ is the Fourier transform of $J(x)$. Expression in (4.11) is therefore estimated as follows

$$
\left|\int_{\mathbb{T}}\langle\hat{\psi}(k)\rangle_{\mu_{\epsilon}} \hat{J}\left(\frac{k}{\epsilon}\right) d k\right| \leq\left[\int_{\mathbb{T}} d k\left\langle|\hat{\psi}(k)|^{2}\right\rangle_{\mu_{\epsilon}}^{2}\right]^{1 / 4}\left[\int_{\mathbb{T}}\left|\hat{J}\left(\frac{k}{\epsilon}\right)\right|^{4 / 3} d k\right]^{3 / 4},
$$

where the estimate follows by Hölder inequality. Using the change of variables $k^{\prime}:=$ $k / \epsilon$ in the second integral on the right hand side we conclude that it is bounded by $\left(\epsilon K_{1}\right)^{1 / 4}\|\hat{J}\|_{L^{4 / 3}(\mathbb{R})}$ for $\epsilon \in(0,1]$, which proves (4.10).

4.2. Homogeneous random fields on $\mathbb{Z}$. Suppose that $\mathcal{E}: \mathbb{T} \rightarrow[0,+\infty)$ is a Borel measurable function such that

$$
\sum_{x \in \mathbb{Z}}\left|\int_{\mathbb{T}} \mathcal{E}(k) e^{2 \pi i k x} d k\right|<+\infty
$$

Let $\left(\xi_{y}\right)_{y \in \mathbb{Z}}$ be a sequence of i.i.d. complex Gaussian random variables such that $\mathbb{E} \xi_{0}=0$ and $\mathbb{E}\left|\xi_{0}\right|^{2}=1$. Define

$$
\hat{\psi}(k)=\sum_{x \in \mathbb{Z}} \xi_{x} \mathcal{E}^{1 / 2}(k) e^{-2 \pi i k x}
$$

a Gaussian, random $H^{-m}(\mathbb{T})$-valued element, where $m>1 / 2$. Its covariance field equals 


$$
\mathcal{C}\left(J_{1}, J_{2}\right):=\mathbb{E}\left[\left\langle J_{1}, \hat{\psi}\right\rangle\left\langle J_{2}, \hat{\psi}\right\rangle^{*}\right]=\int_{\mathbb{T}} \mathcal{E}(k) J_{1}(k) J_{2}^{*}(k) d k
$$

for any $J_{1}, J_{2} \in C^{\infty}(\mathbb{T})$. Then,

$$
\psi_{x}:=\int_{\mathbb{T}} e^{2 \pi i k x} \hat{\psi}(k) d k, \quad x \in \mathbb{Z},
$$

is a complex Gaussian, stationary field. Function $\mathcal{E}(k)$ is called the spectral measure of the field $\left(\psi_{x}\right)_{x \in \mathbb{Z}}$. In the particular case when $\mathcal{E}(k) \equiv 2 \mathcal{E}_{0}$ we denote by $\mu_{\mathcal{E}_{0}}$ the law of the respective field $\left(\psi_{x}\right)_{x \in \mathbb{Z}}$. It is supported in $h_{-m}$, if $m>1 / 2$. One can verify that $\left(\psi_{x}\right)_{x \in \mathbb{Z}}$ satisfies

$$
\left\langle\psi_{x}\right\rangle_{\mu_{\mathcal{E}_{0}}}=0, \quad\left\langle\psi_{x} \psi_{x^{\prime}}\right\rangle_{\mu_{\mathcal{E}_{0}}}=0, \quad\left\langle\psi_{x}^{*} \psi_{x^{\prime}}\right\rangle_{\mu_{\mathcal{E}_{0}}}=2 \mathcal{E}_{0} \delta_{x, x^{\prime}}, \quad x, x^{\prime} \in \mathbb{Z} .
$$

\section{Finite Macroscopic Energy: Initial Data in $L^{2}$}

5.1. The wave function and its evolution. The wave function, adjusted to the macroscopic time, is defined as (see [5])

$$
\psi_{x}^{(\epsilon)}(t):=\left(\tilde{\omega} * \mathfrak{q}\left(\epsilon^{-\delta} t\right)\right)_{x}+i \mathfrak{p}_{x}\left(\epsilon^{-\delta} t\right), \quad x \in \mathbb{Z},
$$

where $\left(\mathfrak{p}_{x}(t), \mathfrak{q}_{x}(t)\right)_{x \in \mathbb{Z}}$ satisfies $(2.5)$ and $\delta \in[0,2]$ is to be chosen later. Function $\left(\tilde{\omega}_{x}\right)_{x \in \mathbb{Z}}$ is the inverse Fourier transform of the dispersion relation function $\omega(k):=$ $\sqrt{\hat{\alpha}(k)}$. The Fourier transform of the wave function is given by

$$
\hat{\psi}^{(\epsilon)}(t, k)=\omega(k) \hat{\mathfrak{q}}\left(\frac{t}{\epsilon^{\delta}}, k\right)+i \hat{\mathfrak{p}}\left(\frac{t}{\epsilon^{\delta}}, k\right), \quad k \in \mathbb{T} .
$$

Since $\mathfrak{p}_{x}(t), \mathfrak{q}_{x}(t)$ are real valued we have

$$
\left(\hat{\psi}^{(\epsilon)}\right)^{*}(t,-k)=\omega(k) \hat{\mathfrak{q}}\left(\frac{t}{\epsilon^{\delta}}, k\right)-i \hat{\mathfrak{p}}\left(\frac{t}{\epsilon^{\delta}}, k\right) .
$$

From (2.5) we conclude that $\left(\hat{\psi}^{(\epsilon)}(t)\right)_{t \geq 0}$ is an $L^{2}(\mathbb{T})$-valued, adapted process that is the unique solution of the Itô stochastic differential equation, understood in the mild sense (see e.g. Theorem 7.4 of [9])

$$
\begin{aligned}
d \hat{\psi}^{(\epsilon)}(t, k)= & \left\{\frac{-i \omega(k)}{\epsilon^{\delta}} \hat{\psi}^{(\epsilon)}(t, k)-\frac{\gamma R(k)}{\epsilon^{\delta}}\left[\hat{\psi}^{(\epsilon)}(t, k)-\left(\hat{\psi}^{(\epsilon)}\right)^{*}(t,-k)\right]\right\} d t \\
& +\frac{i \gamma^{1 / 2}}{\epsilon^{\delta / 2}} \int_{\mathbb{T}} r\left(k, k^{\prime}\right)\left[\hat{\psi}^{(\epsilon)}\left(t, k-k^{\prime}\right)-\left(\hat{\psi}^{(\epsilon)}\right)^{*}\left(t, k^{\prime}-k\right)\right] B\left(d t, d k^{\prime}\right),
\end{aligned}
$$

where $\hat{\psi}^{(\epsilon)}(0) \in L^{2}(\mathbb{T}), R(k)=\hat{\beta}(k) / 4$, and

$$
r\left(k, k^{\prime}\right):=2 \mathfrak{s}^{2}(k) \mathfrak{s}\left(2\left(k-k^{\prime}\right)\right)+2 \mathfrak{s}(2 k) \mathfrak{s}^{2}\left(k-k^{\prime}\right), \quad k, k^{\prime} \in \mathbb{T} .
$$

The process $B(d t, d k)$ is a cylindrical Wiener noise on $L^{2}(\mathbb{T})$ given by

$$
B(d t, d k)=\sum_{x \in \mathbb{Z}} w_{x}(d t) e_{x}^{*}(k) d k,
$$

where $\left(w_{x}\right)$ are i.i.d. standard, 1-dimensional real Brownian motions. 
5.2. Asymptotics of the Wigner transform. In what follows we assume that condition (4.3) holds. Suppose also that $s \in[0,1)$ and that $\gamma=\gamma_{0} \epsilon^{s}$. The noise in (2.5) is called weak (resp. strong) if $s>0$ (resp. $s=0$ ). Furthermore assume that for any $J \in \mathcal{S}$ such that $J(y, k) \equiv J(y)$ we have

$$
\lim _{\epsilon \rightarrow 0+} W_{\epsilon}^{(0)}(J)=\int_{\mathbb{R}} W_{0}(y) J(y) d y,
$$

where $W_{0}(\cdot)$ belongs to $L^{1}(\mathbb{R})$ and is non-negative. Its Fourier transform shall be denoted by

$$
\bar{W}_{0}(p):=\int_{\mathbb{R}} e^{-2 \pi i p y} W_{0}(y) d y .
$$

Since the total energy of the system $\sum_{x \in \mathbb{Z}}\left|\psi_{x}(t)\right|^{2}$ is conserved in time, see Section 2 of [5], for each $\epsilon \in(0,1]$ we have

$$
\left\|\hat{\psi}^{(\epsilon)}(t)\right\|_{L^{2}(\mathbb{T})}=\|\hat{\psi}\|_{L^{2}(\mathbb{T})}, \quad t \geq 0, \quad \mathbb{P}_{\epsilon} \text { a.s. }
$$

Let $W_{\epsilon}(t)$ be the (averaged) Wigner transform of $\psi^{(\epsilon)}(t)$ given by

$$
\left\langle W_{\epsilon}(t), J\right\rangle:=\frac{\epsilon}{2} \int_{\mathbb{R} \times \mathbb{T}} \mathbb{E}_{\epsilon}\left[\left(\hat{\psi}^{(\epsilon)}\right)^{*}\left(t, k-\frac{\epsilon p}{2}\right) \hat{\psi}^{(\epsilon)}\left(t, k+\frac{\epsilon p}{2}\right)\right] \hat{J}^{*}(p, k) d p d k .
$$

Here, as we recall, $\mathbb{E}_{\epsilon}$ is the expectation with respect to $\mathbb{P}_{\epsilon}=\mu_{\epsilon} \otimes \mathbb{P}$. From (5.8) we conclude, thanks to (4.7), that

$$
\sup _{\epsilon \in(0,1]} \sup _{t \geq 0}\left\|W_{\epsilon}(t)\right\|_{\mathcal{A}^{\prime}} \leq K_{0},
$$

where $K_{0}$ is the constant appearing in condition (4.6). As a direct consequence of the above estimate we infer that the family $\left(W_{\epsilon}(\cdot)\right)_{\epsilon \in(0,1]}$ is $*$-weakly sequentially compact in any $L^{\infty}\left([0, T] ; \mathcal{A}^{\prime}\right)$, where $T>0$.

Our main result states that, given $s \in[0,1)$, the exponent $\delta$ can be adjusted so that $\left(W_{\epsilon}(\cdot)\right)$ is *-weakly convergent, as $\epsilon \rightarrow 0+$, in any $L^{\infty}\left([0, T] ; \mathcal{A}^{\prime}\right)$, where $T>0$. The cases of pinned $(\hat{\alpha}(0)>0)$ and unpinned chains $(\hat{\alpha}(0)=0)$ are considered in Sects. 5.2.1 and 5.2.2 respectively. Before presenting our results let us recall briefly the case of the kinetic limit treated in [5], see Theorem 5 in ibid., corresponding to $s=1$, which is outside of the scope of our results. Then, taking $\delta=1$ the family $W_{\epsilon}(\cdot)$ is ${ }^{*}$-weakly convergent, as $\epsilon \rightarrow 0+$, to the unique weak solution of the linear kinetic equation

$$
\partial_{t} W(t, y, k)+\frac{\omega^{\prime}(k)}{2 \pi} \partial_{y} W(t, y, k)=\gamma_{0} \mathcal{L} W(t, y, k) .
$$

The scattering operator $\mathcal{L}$, acting on the $k$-variable, is defined by

$$
\mathcal{L} w(k):=2 \int_{\mathbb{T}} R\left(k, k^{\prime}\right) w\left(k^{\prime}\right) d k^{\prime}-2 R(k) w(k), \quad w \in L^{1}(\mathbb{T}) .
$$

Here $R(k)$ is given by (3.18) and

$$
R\left(k, k^{\prime}\right):=\frac{3}{4} \sum_{\iota \in\{-,+\}} \mathfrak{e}_{\iota}(k) \mathfrak{e}_{-\iota}\left(k^{\prime}\right),
$$


with

$$
\mathfrak{e}_{+}(k):=\frac{8}{3} \mathfrak{s}^{4}(k), \quad \mathfrak{e}_{-}(k):=2 \mathfrak{s}^{2}(2 k)
$$

Note that

$$
R(k)=\int_{\mathbb{T}} R\left(k, k^{\prime}\right) d k^{\prime}=\frac{3}{4} \sum_{\iota \in\{-,+\}} \mathfrak{e}_{\iota}(k) .
$$

From (2.7) we conclude that

$$
R(k) \approx \sin ^{2}(\pi k), \quad k \in \mathbb{T} .
$$

5.2.1. Case of a pinning potential-diffusive transport of energy. Suppose that

$$
\hat{\alpha}(0)=\sum_{x} \alpha_{x}>0 \text {. }
$$

Since (5.17) together with the assumption $\hat{\alpha}^{\prime \prime}(0)>0$ imply that

$$
\left|\omega^{\prime}(k)\right| \approx|\sin (\pi k)|, \quad k \in \mathbb{T} .
$$

From the above and (5.16) we infer that $\hat{\sigma}^{2}$ given by formula (3.17) is finite.

Theorem 5.1. Assume that conditions (5.6) and (5.17) are in force and $\delta=2-s$, where $s \in[0,1)$. Then, for any $T>0$ the Wigner transforms $W_{\epsilon}(\cdot)$ converge, as $\epsilon \rightarrow 0+$, in the $*$-weak sense in $L^{\infty}\left([0, T] ; \mathcal{A}^{\prime}\right)$ to $W(\cdot)$ given by

$$
W(t, y):=\int_{\mathbb{R}} e^{2 \pi i p y} \widehat{W}(t, p) d p,
$$

where,

$$
\widehat{W}(t, p)=\exp \left\{-\frac{\hat{c} p^{2} t}{2}\right\} \bar{W}_{0}(p), \quad t \geq 0
$$

and $\hat{c}$ is defined by (3.21), if $s \in(0,1)$ (weaknoise), or by (3.20), if $s=0$ (strong noise).

5.2.2. Case of a no pinning potential-3/2 fractional superdiffusion. Suppose that

$$
\hat{\alpha}(0)=\sum_{x} \alpha_{x}=0 .
$$

Recall that in this case the dispersion relation satisfies (2.4). Therefore, the integral appearing on the right hand side of (3.17) becomes divergent. Define

$$
\widehat{W}(t, p)=\exp \left\{-\hat{c}|p|^{3 / 2} t\right\} \bar{W}_{0}(p), \quad t \geq 0,
$$

with $\bar{W}_{0}(p)$ given by (5.7) and

$$
\hat{c}:=\frac{\left[\alpha^{\prime \prime}(0)\right]^{3 / 4}}{2^{9 / 4}\left(3 \gamma_{0}\right)^{1 / 2}} .
$$

Our result can be formulated as follows.

Theorem 5.2. Assume that (5.6) and (5.20) are in force. Then, the convergence assertion made in Theorem 5.1 still holds for any $s \in[0,1)$ and $\delta=(3-s) / 2$. The limit $W(t)$ is given by (5.18) and (5.21).

The proofs of the above two theorems are presented in Sect. 11.2. 
5.3. Energy modes. Thanks to condition $\mathfrak{e}_{x}(t)$ defined in (3.8) are non-negative.

A simple calculation, using the definition of the Wigner transform, see (4.8), shows that

$$
\frac{\epsilon}{2} \sum_{x} J(\epsilon x) \mathbb{E}_{\epsilon}\left|\psi_{x}^{(\epsilon)}(t)\right|^{2}=\left\langle W_{\epsilon}(t), J\right\rangle, \quad J \in C_{0}^{\infty}(\mathbb{R})
$$

Theorem 3.1 (resp. Theorem 3.2) is a consequence of (5.23), Theorem 5.2 (resp. Theorem 5.1) and the following result, proved in Sect. 13.1.

Proposition 5.3. Suppose that $\hat{\alpha}(0)>0($ resp. $\hat{\alpha}(0)=0)$ and that condition (4.3) holds. Then,

$$
\lim _{\epsilon \rightarrow 0+} \epsilon \sum_{x} J(\epsilon x) \mathbb{E}_{\epsilon}\left[\mathfrak{e}_{x}\left(\frac{t}{\epsilon^{\delta}}\right)-\frac{1}{2}\left|\psi_{x}^{(\epsilon)}(t)\right|^{2}\right]=0, \quad t \geq 0, J \in C_{0}^{\infty}(\mathbb{R})
$$

for $\delta$ as in the statement of Theorem 5.1 (resp. Theorem 5.2).

\section{Fluctuations in Equilibrium}

In this section we assume that the system is in equilibrium, i.e. that $\left(\psi_{x}\right)_{x \in \mathbb{Z}}$ is a homogeneous, complex Gaussian random field whose covariance function is given by (4.15). As we have already mentioned, its law $\mu_{\mathcal{E}_{0}}$ is supported in $h_{-m}$ for $m>1 / 2$ and the Fourier transform $\hat{\psi}(k)$ belongs to $H^{-m}(\mathbb{T}), \mu_{\mathcal{E}_{0}}$ a.s.

Define $\left(\psi_{x}^{(\epsilon)}(t)\right)$ as the field given by the Fourier coefficients of the solution $\left(\hat{\psi}^{(\epsilon)}\right.$ $(t, k))$ of the Eq. (5.4) whose initial data is distributed according to $\mu_{\mathcal{E}_{0}}$. It has been shown in [12], see Proposition 2.1, that there exists a unique solution of the equation, understood in the mild sense in $C\left([0,+\infty) ; H^{-m}(\mathbb{T})\right)$ for any $m>1 / 2$, in case $\omega(0)>0$ and for $m \in(1 / 2,3 / 2)$, in the unpinned case. Furthermore, see Section 5.1 of ibid., the law of $\hat{\psi}$ in $H^{-m}(\mathbb{T})$ is invariant in time under the dynamics determined by (5.4).

6.1. Fluctuating Wigner distribution. For a given $J \in \mathcal{S}$ define the random Wigner transform as the field

$$
\widetilde{\mathcal{W}}_{\epsilon}(\psi ; J):=\frac{\sqrt{\epsilon}}{2} \sum_{x, x^{\prime} \in \mathbb{Z}}\left(\psi_{x^{\prime}}^{*} \psi_{x}-2 \delta_{x, x^{\prime}} \mathcal{E}_{0}\right) \tilde{J}^{*}\left(\frac{\epsilon}{2}\left(x+x^{\prime}\right), x^{\prime}-x\right),
$$

where

$$
\tilde{J}(y, x)=\int_{\mathbb{T}} e^{2 \pi i x k} J(y, k) d k, \quad(y, x) \in \mathbb{R} \times \mathbb{Z} .
$$

We will also denote $\widetilde{\mathcal{W}}_{\epsilon}(t ; J):=\widetilde{\mathcal{W}}_{\epsilon}\left(\psi^{(\epsilon)}(t) ; J\right)$. From the time invariance of the law of $\psi^{(\epsilon)}(t)$ and (4.15) we obtain

$$
\mathbb{E} \widetilde{\mathcal{W}}_{\epsilon}(t ; J) \equiv\left\langle\widetilde{\mathcal{W}}_{\epsilon}(0 ; J)\right\rangle_{\mathcal{E}_{0}}=0, \quad t \geq 0
$$

Given $J_{1}, J_{2} \in \mathcal{S}$ define also the covariance field

$$
C_{\epsilon}\left(t ; J_{1}, J_{2}\right):=\mathbb{E}\left[\widetilde{\mathcal{W}}_{\epsilon}\left(t ; J_{1}\right) \tilde{\mathcal{W}}_{\epsilon}\left(0 ; J_{2}\right)^{*}\right] .
$$


In the particular case when $t=0$ we obtain

$$
\begin{aligned}
C_{\epsilon}\left(0 ; J_{1}, J_{2}\right) & =\left\langle\widetilde{\mathcal{W}}_{\epsilon}\left(0 ; J_{1}\right) \widetilde{\mathcal{W}}_{\epsilon}\left(0 ; J_{2}\right)^{*}\right\rangle_{\mathcal{E}_{0}} \\
& =\mathcal{E}_{0}^{2} \epsilon \sum_{x, x^{\prime}} \tilde{J}_{1}^{*}\left(\frac{\epsilon\left(x+x^{\prime}\right)}{2}, x^{\prime}-x\right) \tilde{J}_{2}\left(\frac{\epsilon\left(x+x^{\prime}\right)}{2}, x^{\prime}-x\right) .
\end{aligned}
$$

Using the Parseval identity we conclude that

$$
\lim _{\epsilon \rightarrow 0+} C_{\epsilon}\left(0 ; J_{1}, J_{2}\right)=\mathcal{E}_{0}^{2} \int_{\mathbb{R} \times \mathbb{T}} J_{1}^{*}(y, k) J_{2}(y, k) d y d k,
$$

for any $J_{1}, J_{2} \in \mathcal{S}$ and in the case $J_{m}(y, k) \equiv J_{m}(y), m=1,2$ we have

$$
\lim _{\epsilon \rightarrow 0+} C_{\epsilon}\left(0 ; J_{1}, J_{2}\right)=\mathcal{E}_{0}^{2} \int_{\mathbb{R}} J_{1}(y) \tilde{J}_{2}^{*}(y) d y .
$$

\subsection{Statements of the results.}

6.2.1. Case of a pinning potential. Recall, see (5.7), that

$$
\bar{J}(p):=\int_{\mathbb{T}} \widehat{J}(p, k) d k, \quad J \in \mathcal{S} .
$$

Our result dealing with this situation can be formulated as follows.

Theorem 6.1. If $\hat{\alpha}(0)>0$ and $\delta=2-s$, where $s \in[0,1)$ then,

$$
\begin{aligned}
& \lim _{\epsilon \rightarrow 0+} \int_{0}^{+\infty} \phi(t) C_{\epsilon}\left(t ; J_{1}, J_{2}\right) d t \\
& =\mathcal{E}_{0}^{2} \int_{0}^{+\infty} \int_{\mathbb{R}} \exp \left\{-\frac{\hat{c} p^{2} t}{2}\right\} \phi(t) \bar{J}_{1}(p) \bar{J}_{2}(p) d t d p,
\end{aligned}
$$

for any $\phi \in L^{1}[0,+\infty), J_{1}, J_{2} \in \mathcal{S}$. Here $\hat{c}$ is as in Theorem 3.2.

6.2.2. Case of a no pinning potential. The result in this case can be formulated as follows.

Theorem 6.2. If $\hat{\alpha}(0)=0$ and $\delta=(3-s) / 2$, where $s \in[0,1)$, then

$$
\begin{aligned}
& \lim _{\epsilon \rightarrow 0+} \int_{0}^{+\infty} \phi(t) C_{\epsilon}\left(t ; J_{1}, J_{2}\right) d t \\
& =\mathcal{E}_{0}^{2} \int_{0}^{+\infty} \int_{\mathbb{R}} \exp \left\{-\hat{c}|p|^{3 / 2} t\right\} \phi(t) \bar{J}_{1}(p) \bar{J}_{2}(p) d t d p,
\end{aligned}
$$

for any $\phi \in L^{1}[0,+\infty), J_{1}, J_{2} \in \mathcal{S}$. Here $\hat{c}$ is given by (3.21).

The proofs Theorems 6.1 and 6.2 are presented in Sect. 12.

6.2.3. Energy fluctuations. Applying the Wigner fluctuating field to a function $J(y)$ constant in $k$ we obtain the fluctuation field 


$$
\widetilde{W}_{\epsilon}(t ; J)=\sqrt{\epsilon} \sum_{x}\left(\frac{1}{2}\left|\psi_{x}^{(\epsilon)}(t)\right|^{2}-\mathcal{E}_{0}\right) J(\epsilon x) .
$$

Denote the empirical fluctuation of energy field by

$$
\mathcal{E}_{\epsilon}(t ; J):=\sqrt{\epsilon} \sum_{x}\left(\mathfrak{e}_{x}\left(\frac{t}{\epsilon^{\delta}}\right)-\mathcal{E}_{0}\right) J(\epsilon x), \quad J \in C_{0}^{\infty}(\mathbb{R})
$$

and the respective second mixed moment by

$$
C_{\epsilon}^{(e)}\left(t ; J_{1}, J_{2}\right):=\mathbb{E}\left[\mathcal{E}_{\epsilon}\left(t ; J_{1}\right) \mathcal{E}_{\epsilon}\left(0 ; J_{2}\right)\right], \quad J_{1}, J_{2} \in C_{0}^{\infty}(\mathbb{R}) .
$$

Our next result shows the fields defined by (6.8) and (6.9) are asymptotically equal. Its proof is presented in Sect. 13.2.

Proposition 6.3. For any $t \geq 0$ we have

$$
\lim _{\epsilon \rightarrow 0+} \mathbb{E}\left[\mathcal{E}_{\epsilon}(t, J)-\widetilde{\mathcal{W}}_{\epsilon}(t ; J)\right]^{2}=0, \quad J \in C_{0}^{\infty}(\mathbb{R})
$$

As a result the conclusions of Theorems 6.1 and 6.2 hold for $C_{\epsilon}^{(e)}\left(t ; J_{1}, J_{2}\right)$ substituted in place of $C_{\epsilon}\left(t ; J_{1}, J_{2}\right)$, which in turn implies Theorem 3.3.

\section{Outline of the Proofs of Theorems 5.1 and 5.2}

This section is intended to outline the proof of Theorem 5.2 (Theorem 5.1 follows from a similar consideration). First, in Sect. 8, we describe the evolution of the Wigner transform $W_{\epsilon}(t, y, k)$ of the wave function $\psi_{x}^{(\epsilon)}(t)$ introduced in Sect. 5. In fact, for our purposes it is more convenient to deal with its Fourier transform in the spatial domain, given by (8.1). It satisfies the following equation

$$
\begin{aligned}
\partial_{t} \widehat{W}_{\epsilon}(t, p, k)= & \left(-\frac{i \omega^{\prime}(k) p}{\epsilon^{\delta-1}}+\frac{\gamma}{\epsilon^{\delta}} \mathcal{L}\right) \widehat{W}_{\epsilon}(t, p, k) \\
& +\frac{i \gamma R^{\prime}(k) p}{\epsilon^{\delta-1}} \widehat{U}_{\epsilon,-}(t, p, k)-\frac{\gamma}{\epsilon^{\delta}} \mathcal{L} \widehat{U}_{\epsilon,+}(t, p, k)+O(\epsilon)
\end{aligned}
$$

Here, $\gamma=\gamma_{0} \epsilon^{s}$ for some $s \in[0,1)$, with $\delta=(3-s) / 2$ and $O(\epsilon)$ is some expression that becomes negligible, as $\epsilon \rightarrow 0+$. Here $\widehat{U}_{\epsilon,+}(t, p, k)$ represents the difference between the kinetic and potential energy, while $\widehat{U}_{\epsilon,-}(t, p, k)$ is related to the energy current (the product of the momentum and inter-particle distance). They are highly oscillatory and their averages (in time and in $k$ ) turn out to vanish in the limit as $\epsilon \rightarrow 0+$.

To simplify the presentation we assume also here that the scattering kernel equals $R\left(k, k^{\prime}\right)=R(k) R\left(k^{\prime}\right)$, where $R(k)=2 \sin ^{2}(\pi k)$, which is actually the case for the random momentum exchange model described in Sect. 2.1.3. The scattering operator (see (5.12)) is then of the form

$$
\mathcal{L} f(k)=2 R(k)\langle f, R\rangle_{L^{2}(\mathbb{T})}-2 R(k) f(k) .
$$

Since the wave function at time $t=0$ is $L^{2}$ bounded, see (4.6), this bound persists in time, due to the energy conservation. In turn this implies the bound on the norm of $\left(W_{\epsilon}(\cdot)\right)$ in $L^{\infty}\left([0,+\infty) ; \mathcal{A}^{\prime}\right)$, see (5.10). In consequence this family is compact in the 
*-weak topology in $L^{\infty}\left([0, T] ; \mathcal{A}^{\prime}\right)$ for any $T>0$. Our goal is to identify its limit as the function $W(t)$ appearing in the statement of Theorem 5.2. To do so we modify the argument put forward in [18]. To further simplify our presentation we drop the negligible term appearing on the right hand side of (7.1). Performing the Laplace transform on both sides of (7.1) and using the formula (7.2) for the scattering operator $\mathcal{L}$ we obtain that (dropping the arguments $(\lambda, p, k)$ to abbreviate the notation)

$$
\begin{gathered}
\left(\lambda+\frac{2 \gamma}{\epsilon^{\delta}} R+\frac{i \omega^{\prime} p}{\epsilon^{\delta-1}}\right) \bar{w}_{\epsilon}-\widehat{W}_{0}^{(\epsilon)} \approx \frac{2 \gamma R}{\epsilon^{\delta}}\left\langle\bar{w}_{\epsilon}, R\right\rangle_{L^{2}(\mathbb{T})} \\
+\frac{i \gamma R^{\prime} p}{\epsilon^{\delta-1}} \bar{u}_{\epsilon,-}+\frac{2 \gamma R}{\epsilon^{\delta}} \bar{u}_{\epsilon,+}-\frac{2 \gamma R}{\epsilon^{\delta}}\left\langle\bar{u}_{\epsilon,+}, R\right\rangle_{L^{2}(\mathbb{T})}
\end{gathered}
$$

Here $\bar{w}_{\epsilon}(\lambda, p, k)$ and $\bar{u}_{\epsilon, \pm}(\lambda, p, k)$ are the Laplace transforms of $\widehat{W}_{\epsilon}(t, p, k)$ and $\widehat{U}_{\epsilon, \pm}$ $(t, p, k)$, respectively, see (10.5). After some simple computations we get

$$
\bar{w}_{\epsilon}-\frac{2 \gamma R}{D^{(\epsilon)}}\left\langle\bar{w}_{\epsilon}, R\right\rangle_{L^{2}(\mathbb{T})}-\frac{\epsilon^{\delta} \widehat{W}_{0}^{(\epsilon)}}{D^{(\epsilon)}} \approx \frac{i \gamma \epsilon R^{\prime} p}{D^{(\epsilon)}} \bar{u}_{\epsilon,-}+\frac{2 \gamma R}{D^{(\epsilon)}} \bar{u}_{\epsilon,+}-\frac{2 \gamma R}{D^{(\epsilon)}}\left\langle\bar{u}_{\epsilon,+}, R\right\rangle_{L^{2}(\mathbb{T})},
$$

where $D^{(\epsilon)}:=\lambda \epsilon^{\delta}+2 \gamma R+i \epsilon \omega^{\prime}$. Performing the scalar product of both sides of the equation against $2 \gamma R / \epsilon^{\delta}$ we conclude

$$
a_{w}^{(\epsilon)}\left\langle\bar{w}_{\epsilon}, R\right\rangle_{L^{2}(\mathbb{T})}-\int_{\mathbb{T}} \frac{2 \gamma R \widehat{W}_{0}^{(\epsilon)}}{D^{(\epsilon)}} d k \approx \mathcal{O}_{\epsilon}
$$

where $\widehat{W}_{0}^{(\epsilon)}$ is the Fourier-Wigner transform of the initial condition and

$$
a_{w}^{(\epsilon)}:=\frac{2 \gamma}{\epsilon^{\delta}}\left(1-\int_{\mathbb{T}} \frac{2 \gamma R^{2}}{D^{(\epsilon)}} d k\right) .
$$

Here $\mathcal{O}_{\epsilon}$ is the expression that arises from the scalar multiplication of the right hand side of (7.4).

It is quite simple to show that the second term on the left hand side of (7.5) tends to $\bar{W}_{0}(p)$, given by (5.7). Our main effort goes into proving that the right hand side of (7.5) vanishes as $\epsilon \rightarrow 0+$ and that

$$
a_{w}^{(\epsilon)} \rightarrow \lambda+C|p|^{3 / 2}
$$

for an appropriate $C>0$, as $\epsilon \rightarrow 0+$, when $\omega(0)=0$ (we have $a_{w}^{(\epsilon)} \rightarrow \lambda+C p^{2}$ in the unpinned case). The first fact is a consequence of the aforementioned oscillatory behavior of $\bar{u}_{\epsilon, \pm}$, while the convergence of $a_{w}^{(\epsilon)}$ follows from detailed calculations, see Proposition 11.1. This allows us to conclude that $\langle w(\lambda, p), R\rangle_{L^{2}(\mathbb{T})}$ - the limit of $\left\langle w_{\epsilon}(\lambda, p), R\right\rangle_{L^{2}(\mathbb{T})}$, as $\epsilon \rightarrow 0+$, satisfies

$$
\left(\lambda+C|p|^{3 / 2}\right)\langle w(\lambda, p), R\rangle_{L^{2}(\mathbb{T})}=\bar{W}_{0}(p) .
$$

Since in the macroscopic time the number of random collisions grows as $\epsilon^{s-\delta} \gg 1$ (recall that is proportional to $\left.\gamma / \epsilon^{\delta} \sim \epsilon^{s-\delta}\right)$ the limit $w(\lambda, p, k)$ of energy density $\bar{w}_{\epsilon}(\lambda, p, k)$ for a fixed $p$, as $\epsilon \rightarrow 0+$, should become independent of the $k$-variable, therefore, since $\int_{\mathbb{T}} R(k) d k=1$, we ought to have

$$
w(\lambda, p, k) \equiv w(\lambda, p)=\langle w(\lambda, p), R\rangle_{L^{2}(\mathbb{T})} .
$$


This homogenization result is proved in Theorem 10.2 and allows us to conclude (7.7). By virtue of (7.6) and the uniqueness property of the Laplace transform we infer that $W(t)$, appearing in the statement of Theorem 5.2, satisfies (5.21). The "true" argument is a bit more involved, due to the fact that the scattering kernel $R\left(k, k^{\prime}\right)$ corresponding to the noise considered in this paper is not of a product type, see (5.13), which complicates the actual calculations.

\section{Evolution of the Wigner Transform}

For a given $\epsilon>0$ let $\hat{\psi}^{(\epsilon)}(t)$ be a solution of (5.4) with the initial condition $\hat{\psi}$ distributed according to a probability measure $\mu_{\epsilon}$ on $L^{2}(\mathbb{T})$. The Fourier transform of the Wigner transform of $\hat{\psi}^{(\epsilon)}(t)$ is given by

$$
\widehat{W}_{\epsilon}(t, p, k):=\frac{\epsilon}{2} \mathbb{E}_{\epsilon}\left[\left(\hat{\psi}^{(\epsilon)}\right)^{*}\left(t, k-\frac{\epsilon p}{2}\right) \hat{\psi}^{(\epsilon)}\left(t, k+\frac{\epsilon p}{2}\right)\right],
$$

where, as we recall, $\mathbb{E}_{\epsilon}$ is the average with respect to the initial condition and the realization of the noise. To close the equations governing the dynamics of $\widehat{W}_{\epsilon}(t, p, k)$ we shall also need the following functions

$$
\begin{aligned}
& \widehat{Y}_{\epsilon}(t, p, k):=\frac{\epsilon}{2} \mathbb{E}_{\epsilon}\left[\hat{\psi}^{(\epsilon)}\left(t,-k+\frac{\epsilon p}{2}\right) \hat{\psi}^{(\epsilon)}\left(t, k+\frac{\epsilon p}{2}\right)\right], \\
& \widehat{Y}_{\epsilon,-}(t, p, k):=\widehat{Y}_{\epsilon}^{*}(t,-p, k), \quad \widehat{W}_{\epsilon,-}(t, p, k):=\widehat{W}_{\epsilon}(t, p,-k) .
\end{aligned}
$$

We shall also write $\widehat{W}_{\epsilon,+}=\widehat{W}_{\epsilon}$ and $\widehat{Y}_{\epsilon,+}=\widehat{Y}_{\epsilon}$. Define (cf (5.5))

$$
R\left(k, k^{\prime}, p\right):=\frac{1}{2} \sum_{\sigma= \pm 1} r\left(k-\frac{p}{2}, k-\sigma k^{\prime}\right) r\left(k+\frac{p}{2}, k-\sigma k^{\prime}\right) .
$$

With the above definition we can write (see (3.18) for definition $R(\cdot)$ )

$$
\begin{aligned}
\partial_{t} \widehat{W}_{\epsilon}(t, p, k)= & -\left[\frac{i}{\epsilon^{\delta-1}} \delta_{\epsilon} \omega(k ; p)+\frac{2 \gamma \bar{R}(k, \epsilon p)}{\epsilon^{\delta}}\right] \widehat{W}_{\epsilon}(t, p, k) \\
& +\frac{\gamma}{\epsilon^{\delta}}\left\{R\left(k-\frac{\epsilon p}{2}\right) \widehat{Y}_{\epsilon}(t, p, k)+R\left(k+\frac{\epsilon p}{2}\right) \widehat{Y}_{\epsilon,-}(t, p, k)\right\} \\
& +\frac{4 \gamma}{\epsilon^{\delta}} \int_{\mathbb{T}} r\left(k-\frac{\epsilon p}{2}, k^{\prime}\right) r\left(k+\frac{\epsilon p}{2}, k^{\prime}\right) \times \\
& \times \mathbb{E}\left[\left(\hat{\mathfrak{p}}^{(\epsilon)}\right)^{*}\left(t, k-k^{\prime}-\frac{\epsilon p}{2}\right) \hat{\mathfrak{p}}^{(\epsilon)}\left(t, k-k^{\prime}+\frac{\epsilon p}{2}\right)\right] d k^{\prime},
\end{aligned}
$$

where $\delta_{\epsilon} \omega(k, p)$ and $\bar{R}(k, p)$ are defined in (4.1) and

$$
\hat{\mathfrak{p}}^{(\epsilon)}(t, k):=\frac{1}{2 i}\left[\hat{\psi}^{(\epsilon)}(t, k)-\left(\hat{\psi}^{(\epsilon)}\right)^{*}(t,-k)\right]
$$

is the Fourier transform of the momentum. Since the latter is real valued, its Fourier transform is complex even. The last term appearing on the right hand side of (8.4) can be replaced by

$$
\begin{aligned}
& \frac{4 \gamma}{\epsilon^{\delta}} \int_{\mathbb{T}} R\left(k, k^{\prime}, \epsilon p\right) \mathbb{E}_{\epsilon}\left[\left(\hat{\mathfrak{p}}^{(\epsilon)}\right)^{*}\left(t, k^{\prime}-\frac{\epsilon p}{2}\right) \hat{\mathfrak{p}}^{(\epsilon)}\left(t, k^{\prime}+\frac{\epsilon p}{2}\right)\right] d k^{\prime} \\
& =\frac{2 \gamma}{\epsilon^{\delta}}\left(\mathcal{R}_{\epsilon p} \widehat{W}_{\epsilon}\right)(t, p, k)-\frac{\gamma}{\epsilon^{\delta}}\left(\mathcal{R}_{\epsilon p} \widehat{Y}_{\epsilon}\right)(t, p, k)-\frac{\gamma}{\epsilon^{\delta}}\left(\mathcal{R}_{\epsilon p} \widehat{Y}_{\epsilon,-}\right)(t, p, k),
\end{aligned}
$$


where

$$
\mathcal{R}_{p} f(k):=\int_{\mathbb{T}} R\left(k, k^{\prime}, p\right) f\left(k^{\prime}\right) d k^{\prime}
$$

Therefore,

$$
\begin{aligned}
\partial_{t} \widehat{W}_{\epsilon}(t, p, k)= & -\frac{i}{\epsilon^{\delta-1}} \delta_{\epsilon} \omega(k ; p) \widehat{W}_{\epsilon}(t, p, k)+\frac{\gamma}{\epsilon^{\delta}} \mathcal{L}_{\epsilon p} \widehat{W}_{\epsilon}(t, p, k) \\
& -\frac{\gamma}{2 \epsilon^{\delta}} \sum_{\sigma \in\{-,+\}} \mathcal{L}_{\sigma \epsilon p}^{+} \widehat{Y}_{\epsilon,-\sigma}(t, p, k),
\end{aligned}
$$

and

$$
\begin{aligned}
& \mathcal{L}_{p} f(k):=2 \mathcal{R}_{p} f(k)-2 \bar{R}(k, p) f(k), \\
& \mathcal{L}_{p}^{ \pm} f(k):=2 \mathcal{R}_{p} f(k)-2 R\left(k \pm \frac{p}{2}\right) f(k) .
\end{aligned}
$$

In addition,

$$
\begin{aligned}
\partial_{t} \widehat{Y}_{\epsilon}(t, p, k)= & -\frac{2 i}{\epsilon^{\delta}} \bar{\omega}(k, \epsilon p) \widehat{Y}_{\epsilon}(t, p, k)-\frac{2 \gamma}{\epsilon^{\delta}} \bar{R}(k, \epsilon p) \widehat{Y}_{\epsilon}(t, p, k) \\
& +\frac{\gamma}{\epsilon^{\delta}} R\left(k-\frac{\epsilon p}{2}\right) \widehat{W}_{\epsilon}(t, p, k)+\frac{\gamma}{\epsilon^{\delta}} R\left(k+\frac{\epsilon p}{2}\right) \widehat{W}_{\epsilon,-}(t, p, k) \\
& +\frac{\gamma}{\epsilon^{\delta}} \mathcal{U}_{\epsilon}(t, p, k)
\end{aligned}
$$

where

$$
\begin{aligned}
& \mathcal{U}_{\epsilon}(t, p, k):=4 \int_{\mathbb{T}} r\left(-k+\frac{\epsilon p}{2}, k^{\prime}\right) r\left(k+\frac{\epsilon p}{2},-k^{\prime}\right) \\
& \times \mathbb{E}_{\epsilon}\left[\mathfrak{p}^{(\epsilon)}\left(t,-k-k^{\prime}+\frac{\epsilon p}{2}\right) \mathfrak{p}^{(\epsilon)}\left(t, k+k^{\prime}+\frac{\epsilon p}{2}\right)\right] d k .
\end{aligned}
$$

After straightforward calculations (cf (8.4)-(8.7)) we conclude that

$$
\mathcal{U}_{\epsilon}(t, p, k)=\mathcal{R}_{\epsilon p}\left(\widehat{Y}_{\epsilon}+\widehat{Y}_{\epsilon,-}-\widehat{W}_{\epsilon}-\widehat{W}_{\epsilon,-}\right)(t, p, k)
$$

Then,

$$
\begin{aligned}
\partial_{t} \widehat{Y}_{\epsilon}(t, p, k)= & -\frac{2 i}{\epsilon^{\delta}} \bar{\omega}(k, \epsilon p) \widehat{Y}_{\epsilon}(t, p, k)+\frac{\gamma}{\epsilon^{\delta}} \mathcal{L}_{\epsilon p} \widehat{Y}_{\epsilon}(t, p, k) \\
& +\frac{\gamma}{\epsilon^{\delta}} \mathcal{R}_{\epsilon p}\left(\widehat{Y}_{\epsilon,-}-\widehat{Y}_{\epsilon}\right)(t, p, k)-\frac{\gamma}{2 \epsilon^{\delta}} \sum_{\sigma \in\{-,+\}} \mathcal{L}_{\sigma \epsilon p}^{+} \widehat{W}_{\epsilon,-\sigma}(t, p, k) .
\end{aligned}
$$

From (8.7) and (8.10) we conclude that for any fixed $p \in \mathbb{R}$ the evolution $\left(\widehat{W}_{\epsilon}(t)\right.$, $\left.\widehat{Y}_{\epsilon}(t), \widehat{Y}_{\epsilon,-}(t), \widehat{W}_{\epsilon,-}(t)\right)$ is governed by a closed system of four linear equations with a generator that is a bounded operator in $\left(L^{r}(\mathbb{T})\right)^{4}$ for any $r \in[1,+\infty]$. In particular, under the assumption that the initial distribution of the wave functions satisfies (4.3) the components of $\left(\widehat{W}_{\epsilon}(t), \widehat{Y}_{\epsilon}(t), \widehat{Y}_{\epsilon,-}(t), \widehat{W}_{\epsilon,-}(t)\right)$ belong to $C\left([0,+\infty) ; \mathcal{A}^{\prime}\right)$.

\section{Expansion of the Dynamics of the Wigner Transform}

Assumption (4.3) guarantees that

$$
\sum_{l \in\{-,+\}} \sup _{p \in \mathbb{R}}\left(\left\|\widehat{W}_{\epsilon, l}(0, p, \cdot)\right\|_{L^{2}(\mathbb{T})}+\left\|\widehat{Y}_{\epsilon, l}(0, p, \cdot)\right\|_{L^{2}(\mathbb{T})}\right)<+\infty .
$$


From an elementary existence and uniqueness result concerning the dynamics of $\left(\widehat{W}_{\epsilon}(t)\right.$, $\left.\widehat{Y}_{\epsilon}(t), \widehat{Y}_{\epsilon,-}(t), \widehat{W}_{\epsilon,-}(t)\right)$ in $\left(L^{2}(\mathbb{T})\right)^{4}$ we conclude that

$$
\sup _{t \in[0, T]} \sum_{\iota \in\{-,+\}}\left(\left\|\widehat{W}_{\epsilon, l}(t, p, \cdot)\right\|_{L^{2}(\mathbb{T})}+\left\|\widehat{Y}_{\epsilon, l}(t, p, \cdot)\right\|_{L^{2}(\mathbb{T})}\right)<+\infty .
$$

for any $\epsilon, T>0$ and $p \in \mathbb{R}$.

We expand the scattering kernel appearing on the right hand side of (8.7), (8.10) into the powers of $\epsilon$, up to the second order. To abbreviate the notation we shall write

$$
R_{\epsilon}:=R(k)+\frac{(\epsilon p)^{2}}{8} R^{\prime \prime}(k), \quad \bar{\omega}:=\bar{\omega}(k, \epsilon p), \quad \delta_{\epsilon} \omega:=\delta_{\epsilon} \omega(k, p) .
$$

Since $\partial_{p} R\left(k, k^{\prime}, 0\right)=0$ we can write

$$
\begin{aligned}
\partial_{t} \widehat{W}_{\epsilon}= & -\frac{i \delta_{\epsilon} \omega}{\epsilon^{\delta-1}} \widehat{W}_{\epsilon}+\left(\frac{\gamma}{\epsilon^{\delta}} \mathcal{L}+\frac{\gamma p^{2}}{2 \epsilon^{\delta-2}}\left(\delta^{2} \mathcal{L}\right)\right)\left[\widehat{W}_{\epsilon}-\frac{1}{2}\left(\widehat{Y}_{\epsilon}+\widehat{Y}_{\epsilon,-}\right)\right] \\
& +\frac{\gamma R^{\prime} p}{2 \epsilon^{\delta-1}}\left(\widehat{Y}_{\epsilon,-}-\widehat{Y}_{\epsilon}\right)+\epsilon^{3-\delta} \mathfrak{R}_{\epsilon}^{(1)},
\end{aligned}
$$

and

$$
\begin{aligned}
\partial_{t} \widehat{Y}_{\epsilon}= & -\frac{2 i \bar{\omega}}{\epsilon^{\delta}} \widehat{Y}_{\epsilon}+\left(\frac{\gamma}{\epsilon^{\delta}} \mathcal{L}+\frac{\gamma p^{2}}{2 \epsilon^{\delta-2}}\left(\delta^{2} \mathcal{L}\right)\right)\left[\widehat{Y}_{\epsilon}-\frac{1}{2}\left(\widehat{W}_{\epsilon}+\widehat{W}_{\epsilon,-}\right)\right] \\
& +\left(\frac{\gamma}{\epsilon^{\delta}} \mathcal{R}+\frac{\gamma p^{2}}{2 \epsilon^{\delta-2}}\left(\delta^{2} \mathcal{R}\right)\right)\left(\widehat{Y}_{\epsilon,-}-\widehat{Y}_{\epsilon}\right)+\frac{\gamma R^{\prime} p}{2 \epsilon^{\delta-1}}\left(\widehat{W}_{\epsilon,-}-\widehat{W}_{\epsilon}\right)+\epsilon^{3-\delta} \mathfrak{R}_{\epsilon}^{(2)},
\end{aligned}
$$

where $\mathcal{L}, \mathcal{R} f$ are given by (8.8) and (8.6) and

$$
\begin{aligned}
\left(\delta^{2} \mathcal{L}\right) f(k) & :=-\pi^{2} \int_{\mathbb{T}} R_{1}\left(k, k^{\prime}\right) f\left(k^{\prime}\right) d k^{\prime}-\frac{1}{2} R^{\prime \prime}(k) f(k), \\
\left(\delta^{2} \mathcal{R}\right) f(k) & :=\int_{\mathbb{T}} \partial_{p}^{2} R\left(k, k^{\prime}, 0\right) f\left(k^{\prime}\right) d k^{\prime}=-\frac{\pi^{2}}{2} \int_{\mathbb{T}} R_{1}\left(k, k^{\prime}\right) f\left(k^{\prime}\right) d k^{\prime} .
\end{aligned}
$$

Here

$$
R_{1}\left(k, k^{\prime}\right):=-\frac{2}{\pi^{2}} \partial_{p}^{2} R\left(k, k^{\prime}, 0\right)=4 \mathfrak{f}_{+}(k) \mathfrak{f}_{+}\left(k^{\prime}\right)+\mathfrak{f}_{+}(k) \mathfrak{e}_{-}\left(k^{\prime}\right)+3 \mathfrak{f}_{-}(k) \mathfrak{e}_{+}\left(k^{\prime}\right),
$$

with $\mathfrak{e}_{ \pm}(k)$ defined in (5.14) and

$$
\mathfrak{f}_{+}(k):=2 \mathfrak{s}^{2}(k), \quad \mathfrak{f}_{-}(k):=2 \mathfrak{c}^{2}(k) .
$$

Note that

$$
\left(\delta^{2} \mathcal{L}\right) f_{-}(k)=\left(\left(\delta^{2} \mathcal{L}\right) f\right)(-k),
$$

where $f_{-}(k):=f(-k)$. In addition,

$$
\mathfrak{R}_{\epsilon}^{(i)}(t, 0, k) \equiv 0, \quad i=1,2 .
$$


Adopting the convention $\widehat{W}_{\epsilon}=\widehat{W}_{\epsilon,+}, \widehat{Y}_{\epsilon}=\widehat{Y}_{\epsilon,+}$, we can write that for any $M>0$

$$
\left\|\Re_{\epsilon}^{(i)}(t, p, \cdot)\right\|_{L^{2}(\mathbb{T})} \preceq \sum_{l \in\{-,+\}}\left(\left\|\widehat{W}_{\epsilon, l}(t, p, \cdot)\right\|_{L^{2}(\mathbb{T})}+\left\|\widehat{Y}_{\epsilon, l}(t, p, \cdot)\right\|_{L^{2}(\mathbb{T})}\right)
$$

for $i=1,2$ and all $t \geq 0, \epsilon \in(0,1],|p| \leq M$. Define

$$
\begin{aligned}
\widehat{U}_{\epsilon,+}(t, p, k) & :=\frac{1}{2}\left(\widehat{Y}_{\epsilon}+\widehat{Y}_{\epsilon,-}\right)(t, p, k), \\
\widehat{U}_{\epsilon,-}(t, p, k) & :=\frac{1}{2 i}\left(\widehat{Y}_{\epsilon}-\widehat{Y}_{\epsilon,-}\right)(t, p, k) .
\end{aligned}
$$

From (9.4) and (9.5) we get

$$
\begin{gathered}
\partial_{t} \widehat{W}_{\epsilon,+}=-\frac{i \delta_{\epsilon} \omega}{\epsilon^{\delta-1}} \widehat{W}_{\epsilon,+}-\frac{i \gamma R^{\prime} p}{\epsilon^{\delta-1}} \widehat{U}_{\epsilon,-}+\left(\frac{\gamma}{\epsilon^{\delta}} \mathcal{L}+\frac{\gamma p^{2}}{2 \epsilon^{\delta-2}}\left(\delta^{2} \mathcal{L}\right)\right) \\
\times\left(\widehat{W}_{\epsilon,+}-U_{\epsilon,+}\right)+\epsilon^{3-\delta} \overline{\mathfrak{R}}_{\epsilon}^{(1)}, \\
\partial_{t} \widehat{U}_{\epsilon,+}=\frac{2 \bar{\omega}}{\epsilon^{\delta}} \widehat{U}_{\epsilon,-}+\left(\frac{\gamma}{\epsilon^{\delta}} \mathcal{L}+\frac{\gamma p^{2}}{2 \epsilon^{\delta-2}}\left(\delta^{2} \mathcal{L}\right)\right)\left[\widehat{U}_{\epsilon,+}-\frac{1}{2}\left(\widehat{W}_{\epsilon,+}+\widehat{W}_{\epsilon,-}\right)\right]+\epsilon^{3-\delta} \overline{\mathfrak{R}}_{\epsilon}^{(2)}, \\
\partial_{t} \widehat{U}_{\epsilon,-}=-\frac{2 \bar{\omega}}{\epsilon^{\delta}} \widehat{U}_{\epsilon,+}-\frac{2 \gamma}{\epsilon^{\delta}} R_{\epsilon} \widehat{U}_{\epsilon,-}-\frac{i \gamma R^{\prime} p}{2 \epsilon^{\delta-1}}\left(\widehat{W}_{\epsilon,-}-\widehat{W}_{\epsilon,+}\right)+\epsilon^{3-\delta} \bar{\Re}_{\epsilon}^{(3)}, \\
\partial_{t} \widehat{W}_{\epsilon,-}=\frac{i \delta_{\epsilon} \omega}{\epsilon^{\delta-1}} \widehat{W}_{\epsilon,-}+\frac{i \gamma R^{\prime} p}{\epsilon^{\delta-1}} \widehat{U}_{\epsilon,-}+\left(\frac{\gamma}{\epsilon^{\delta}} \mathcal{L}+\frac{\gamma p^{2}}{2 \epsilon^{\delta-2}}\left(\delta^{2} \mathcal{L}\right)\right)\left(\widehat{W}_{\epsilon,-}-\widehat{U}_{\epsilon,+}\right) \\
+\epsilon^{3-\delta} \overline{\mathfrak{R}}_{\epsilon}^{(4)} .
\end{gathered}
$$

From (9.11) we conclude that for any $M>0, i \in\{1,2,3,4\}$

$$
\left\|\bar{\Re}_{\epsilon}^{(i)}(t, p, \cdot)\right\|_{L^{2}(\mathbb{T})} \preceq \sum_{l \in\{-,+\}}\left(\left\|\widehat{W}_{\epsilon, l}(t, p, \cdot)\right\|_{L^{2}(\mathbb{T})}+\left\|\widehat{U}_{\epsilon, l}(t, p, \cdot)\right\|_{L^{2}(\mathbb{T})}\right)
$$

for $t \geq 0$ and $\epsilon \in(0,1],|p| \leq M$.

Let $\mathcal{D}(\phi):=\mathcal{D}(\phi, \phi)$, where

$$
\begin{aligned}
\mathcal{D}(\phi, \psi) & :=\int_{\mathbb{T}}(-\mathcal{L}) \phi(k) \psi^{*}(k) d k \\
& =\int_{\mathbb{T}^{2}} R\left(k, k^{\prime}\right)\left[\phi(k)-\phi\left(k^{\prime}\right)\right]\left[\psi(k)-\psi\left(k^{\prime}\right)\right]^{*} d k d k^{\prime},
\end{aligned}
$$

for any $\phi, \psi \in L^{2}(\mathbb{T})$ and

$$
\mathfrak{E}_{\epsilon}(t, p):=\sum_{l \in\{-,+\}}\left(\frac{1}{2}\left\|\widehat{W}_{\epsilon, l}(t, p)\right\|_{L^{2}(\mathbb{T})}^{2}+\left\|\widehat{U}_{\epsilon, l}(t, p)\right\|_{L^{2}(\mathbb{T})}^{2}\right) .
$$

Taking the scalar products of both sides of equations appearing in (9.13) against the respective $\widehat{W}_{\epsilon, \iota}, \widehat{U}_{\epsilon, \iota}, \iota \in\{-,+\}$ we obtain

$$
\begin{gathered}
\frac{1}{2} \mathfrak{E}_{\epsilon}(t, p)+\frac{\gamma}{\epsilon^{\delta}} \int_{0}^{t} \mathcal{D}\left(\left(\widehat{W}_{\epsilon}-\widehat{U}_{\epsilon,+}\right)(s, p)\right) d s \\
+\frac{2 \gamma}{\epsilon^{\delta}} \int_{0}^{t} d s \int_{\mathbb{T}} R(k)\left|\widehat{U}_{\epsilon,-}(s, p, k)\right|^{2} d k
\end{gathered}
$$




$$
\begin{aligned}
& +2 \epsilon^{1-\delta} \gamma p \int_{0}^{t} d s \int_{\mathbb{T}} R^{\prime}(k) \operatorname{Im}\left(\widehat{U}_{\epsilon,-}^{*} \widehat{W}_{\epsilon,+}\right)(s, p, k) d k \\
= & \frac{1}{2} \mathfrak{E}_{\epsilon}(0, p)+\epsilon^{2-\delta} \int_{0}^{t} \mathfrak{R}_{\epsilon}(s, p) d s,
\end{aligned}
$$

where for any $M>0$ we have

$$
\mathfrak{R}_{\epsilon}(t, p) \preceq \mathfrak{E}_{\epsilon}(t, p), \quad t \geq 0,|p| \leq M, \epsilon \in(0,1] .
$$

Using Young's inequality and the fact that $\left(R^{\prime}(k)\right)^{2} \preceq R(k)$ (see (14.5) below) we conclude that for any $M>0$ there exists $C>0$ such that

$$
\begin{aligned}
& \epsilon^{1-\delta} \gamma p \int_{0}^{t} d s \int_{\mathbb{T}} R^{\prime}(k) \operatorname{Im}\left(\widehat{U}_{\epsilon,-}^{*} \widehat{W}_{\epsilon,+}\right)(s, p, k) d k \\
& \geq-\frac{\gamma}{\epsilon^{\delta}} \int_{0}^{t} d s \int_{\mathbb{T}} R(k)\left|\widehat{U}_{\epsilon,-}(s, p, k)\right|^{2} d k-C \gamma \epsilon^{2-\delta} \int_{0}^{t}\left\|\widehat{W}_{\epsilon,+}(s, p)\right\|_{L^{2}(\mathbb{T})}^{2} d s,
\end{aligned}
$$

for $t \geq 0,|p| \leq M$ and $\epsilon \in(0,1]$. From the above, estimate (9.16), identity (9.15) and Gronwall's inequality we obtain the following.

Proposition 9.1. For any $M>0$ there exists $C_{1}>0$ such that

$$
\mathfrak{E}_{\epsilon}(t, p) \leq \mathfrak{E}_{\epsilon}(0, p) e^{C_{1} \epsilon^{2-\delta} t}, \quad \forall \epsilon \in(0,1],|p| \leq M, t \geq 0
$$

\section{Laplace Transform of System (9.13)}

For any $\lambda>\lambda_{0}^{(\epsilon)}:=C_{1} \epsilon^{2-\delta}\left(C_{1}\right.$ as in Proposition 9.1) we let

$$
\begin{aligned}
& \bar{w}_{\epsilon, l}(\lambda, p, k):=\int_{0}^{+\infty} e^{-\lambda t} \widehat{W}_{\epsilon, l}(t, p, k) d t, \\
& \bar{u}_{\epsilon, l}(\lambda, p, k):=\int_{0}^{+\infty} e^{-\lambda t} \widehat{U}_{\epsilon, l}(t, p, k) d t .
\end{aligned}
$$

Thanks to Proposition 9.1 the above integrals are well defined in $L^{2}(\mathbb{T})$ for $\lambda>\lambda_{0}^{(\epsilon)}$ and $p \in \mathbb{R}$. Let

$$
\lambda_{0}:= \begin{cases}0, & \delta<2 \\ C_{1}, & \delta=2\end{cases}
$$

and $C_{1}$ is as in (9.18). The "remainder" term $\bar{r}_{\epsilon}^{(i)}(\lambda, p, k)$, that is the Laplace transforms of $\overline{\mathfrak{R}}_{\epsilon}^{(i)}(t, p, k)$, has the following property: for any $M>0$ and compact interval $I \subset$ $\left(\lambda_{0},+\infty\right)$

$$
\left\|\bar{r}_{\epsilon}^{(i)}(\lambda, p)\right\|_{L^{2}(\mathbb{T})} \preceq \sum_{l \in\{-,+\}}\left(\left\|\bar{w}_{\epsilon, l}(\lambda, p)\right\|_{L^{2}(\mathbb{T})}+\left\|\bar{u}_{\epsilon, l}(\lambda, p)\right\|_{L^{2}(\mathbb{T})}\right)
$$


for $i=1,2,3,4$ and $\epsilon \in(0,1],|p| \leq M, \lambda \in I$. Therefore, from Proposition 9.1 we conclude that

$$
C_{I}:=\sup _{\epsilon \in(0,1]} \sup _{\lambda \in I,|p| \leq M}\left(\left\|\bar{w}_{\epsilon, l}(\lambda, p)\right\|_{L^{2}(\mathbb{T})}+\sum_{\iota \in\{-,+\}}\left\|\bar{u}_{\epsilon, l}(\lambda, p)\right\|_{L^{2}(\mathbb{T})}\right)<+\infty .
$$

Taking the Laplace transform of the both sides of equations of the system (9.13) we obtain

$$
\begin{aligned}
& \lambda \bar{w}_{\epsilon,+}-\widehat{W}_{\epsilon,+}^{(0)}=-\frac{i \delta_{\epsilon} \omega}{\epsilon^{\delta-1}} \bar{w}_{\epsilon,+}-\frac{i \gamma R^{\prime} p}{\epsilon^{\delta-1}} \bar{u}_{\epsilon,-}+\frac{\gamma}{\epsilon^{\delta}} L_{\epsilon}\left(\bar{w}_{\epsilon,+}-\bar{u}_{\epsilon,+}\right)+\epsilon^{3-\delta} \bar{r}_{\epsilon}^{(1)}, \\
& \lambda \bar{u}_{\epsilon,+}-\widehat{U}_{\epsilon,+}^{(0)}=\frac{2 \bar{\omega}}{\epsilon^{\delta}} \bar{u}_{\epsilon,-}+\frac{\gamma}{\epsilon^{\delta}} L_{\epsilon}\left[\bar{u}_{\epsilon,+}-\frac{1}{2}\left(\bar{w}_{\epsilon,+}+\bar{w}_{\epsilon,-}\right)\right]+\epsilon^{3-\delta} \bar{r}_{\epsilon}^{(2)}, \\
& \lambda \bar{u}_{\epsilon,-}-\widehat{U}_{\epsilon,-}^{(0)}=-\frac{2 \bar{\omega}}{\epsilon^{\delta}} \bar{u}_{\epsilon,+}-\frac{2 \gamma}{\epsilon^{\delta}} R_{\epsilon} \bar{u}_{\epsilon,-}-\frac{i \gamma R^{\prime} p}{2 \epsilon^{\delta-1}}\left(\bar{w}_{\epsilon,-}-\bar{w}_{\epsilon,+}\right)+\epsilon^{3-\delta} \bar{r}_{\epsilon}^{(3)} \\
& \lambda \bar{w}_{\epsilon,-}-\widehat{W}_{\epsilon,-}^{(0)}=\frac{i \delta_{\epsilon} \omega}{\epsilon^{\delta-1}} \bar{w}_{\epsilon,-}+\frac{i \gamma R^{\prime} p}{\epsilon^{\delta-1}} \bar{u}_{\epsilon,-}+\frac{\gamma}{\epsilon^{\delta}} L_{\epsilon}\left(\bar{w}_{\epsilon,-}-\bar{u}_{\epsilon,+}\right)+\epsilon^{3-\delta} \bar{r}_{\epsilon}^{(4)},
\end{aligned}
$$

where $L_{\epsilon}:=\mathcal{L}+(1 / 2)(\epsilon p)^{2}\left(\delta^{2} \mathcal{L}\right)$ and $\mathcal{L}, \delta^{2} \mathcal{L}$ are given by (5.12) and (9.6), respectively. Performing the real parts of the scalar products in $L^{2}(\mathbb{T})$ of the respective equations of the above system with $(1 / 2) \bar{w}_{\epsilon, \pm}(\lambda, p, k), \bar{u}_{\epsilon, \pm}(\lambda, p, k)$ and adding them sideways we get

$$
\begin{aligned}
& \lambda\left(\left\|\bar{w}_{\epsilon}(\lambda, p)\right\|_{L^{2}(\mathbb{T})}^{2}+\sum_{\iota \in\{-,+\}}\left\|\bar{u}_{\epsilon,+}(\lambda, p)\right\|_{L^{2}(\mathbb{T})}^{2}\right) \\
& +\frac{2 \gamma}{\epsilon^{\delta}} \int_{\mathbb{T}} R_{\epsilon}(k)\left|\bar{u}_{\epsilon,-}(\lambda, p, k)\right|^{2} d k+2 \epsilon^{1-\delta} \gamma p \int_{\mathbb{T}} R^{\prime}(k) \operatorname{Im}\left(\bar{u}_{\epsilon,-}^{*} \bar{w}_{\epsilon}\right)(\lambda, p, k) d k \\
& +\frac{\gamma}{\epsilon^{\delta}} \mathcal{D}\left(\left(\bar{w}_{\epsilon}-\bar{u}_{\epsilon,+}\right)(\lambda, p)\right)=\operatorname{Re} \int_{\mathbb{T}} \widehat{W}_{\epsilon}^{(0)}(p, k) \bar{w}_{\epsilon}(\lambda, p, k) d k \\
& +\sum_{\iota \in\{-,+\}} \operatorname{Re} \int_{\mathbb{T}} \widehat{U}_{\epsilon, \iota}^{(0)}(p, k) \bar{u}_{\epsilon, \iota}(\lambda, p, k) d k+\overline{\mathfrak{R}}_{\epsilon}(\lambda, p) .
\end{aligned}
$$

Given $M>0$ and $I \subset\left(\lambda_{0},+\infty\right)$ compact, we have

$$
\overline{\mathfrak{R}}_{\epsilon}(\lambda, p) \preceq\left\|\bar{w}_{\epsilon, l}(\lambda, p)\right\|_{L^{2}(\mathbb{T})}^{2}+\sum_{\iota \in\{-,+\}}\left\|\bar{u}_{\epsilon, l}(\lambda, p)\right\|_{L^{2}(\mathbb{T})}^{2}
$$

for all $\epsilon \in(0,1]$ and $\lambda \in I,|p| \leq M$. Using again Young's inequality, as in (9.17), together with (10.4) we conclude that for any $M>0$, a compact interval $I \subset\left(\lambda_{0},+\infty\right)$ and $\epsilon \in(0,1]$

$$
\sup _{\lambda \in I,|p| \leq M}\left[\int_{\mathbb{T}} R_{\epsilon}(k)\left|\bar{u}_{\epsilon,-}(\lambda, p, k)\right|^{2} d k+\mathcal{D}\left(\left(\bar{w}_{\epsilon}-\bar{u}_{\epsilon,+}\right)(\lambda, p)\right)\right] \preceq \epsilon^{\delta-s} .
$$

In fact it is possible to get a more precise result. 
Proposition 10.1. For any $M>0$ and a compact interval $I \subset\left(\lambda_{0},+\infty\right)$

$$
\begin{aligned}
& \sup _{\lambda \in I,|p| \leq M}\left[\int_{\mathbb{T}} R_{\epsilon}(k)\left|\bar{u}_{\epsilon,-}(\lambda, p, k)\right|^{2} d k+\mathcal{D}\left(\bar{w}_{\epsilon}(\lambda, p)\right)\right. \\
& \left.+\int_{\mathbb{T}} R_{\epsilon}(k)\left|\bar{u}_{\epsilon,+}(\lambda, p, k)\right|^{2}\left(\frac{\bar{\omega}(k, \epsilon p)}{\lambda \epsilon^{\delta}+\gamma R_{\epsilon}(k)}\right)^{2} d k\right] \preceq \epsilon^{\delta-s}, \quad \epsilon \in(0,1] .
\end{aligned}
$$

We postpone the proof of the above Proposition till Sect. 14.2 and use it first to show a homogenization result formulated below. Define

$$
w_{\epsilon}^{(\iota)}(\lambda, p):=\int_{\mathbb{T}} \bar{w}_{\epsilon}(\lambda, p, k) \mathfrak{e}_{\iota}(k) d k, \quad \iota \in\{-,+\}
$$

Theorem 10.2. Suppose that the initial laws $\left(\mu_{\epsilon}\right)$ satisfy (4.3). Then, for any $M>0$ and a compact interval $I \subset\left(\lambda_{0},+\infty\right)$ we have

$$
\lim _{\epsilon \rightarrow 0+} \sup _{\lambda \in I,|p| \leq M} \int_{\mathbb{T}}\left|\bar{w}_{\epsilon}(\lambda, p, k)-w_{\epsilon}^{( \pm)}(\lambda, p)\right| d k=0,
$$

and

$$
\lim _{\epsilon \rightarrow 0+} \sup _{\lambda \in I,|p| \leq M} \int_{\mathbb{T}}\left|\bar{u}_{\epsilon, l}(\lambda, p, k)\right| d k=0, \quad \iota \in\{-,+\} .
$$

Proof. Consider the case $\iota=-$. By (10.4), it is enough to prove that

$$
\lim _{\epsilon \rightarrow 0+} \sup _{\lambda \in I,|p| \leq M} \int_{\mathbb{T}}\left|\bar{w}_{\epsilon}(\lambda, p, k)-w_{\epsilon}^{(-)}(\lambda, p)\right| \mathfrak{e}_{-}(k) d k=0 .
$$

By virtue of (10.8) we have

$$
\begin{aligned}
\int_{\mathbb{T}} \mid & \bar{w}_{\epsilon}(\lambda, p, k)-w_{\epsilon}^{(-)}(\lambda, p) \mid \mathfrak{e}_{-}(k) d k \\
\leq & \int_{\mathbb{T}^{2}}\left|\bar{w}_{\epsilon}(\lambda, p, k)-\bar{w}_{\epsilon}\left(\lambda, p, k^{\prime}\right)\right| \mathfrak{e}_{-}(k) \mathfrak{e}_{-}\left(k^{\prime}\right) d k d k^{\prime} \\
\leq & \left\{\int_{\mathbb{T}^{2}} R\left(k, k^{\prime}\right)\left|\bar{w}_{\epsilon}(\lambda, p, k)-\bar{w}_{\epsilon}\left(\lambda, p, k^{\prime}\right)\right|^{2} d k d k^{\prime}\right\}^{1 / 2} \\
& \times\left\{\int_{\mathbb{T}^{2}} \frac{\mathfrak{e}_{-}^{2}(k) \mathfrak{e}_{-}^{2}\left(k^{\prime}\right)}{R\left(k, k^{\prime}\right)} d k d k^{\prime}\right\}^{1 / 2} \preceq \epsilon^{(\delta-s) / 2} .
\end{aligned}
$$

The last estimate follows from (see (5.13))

$$
\frac{\mathfrak{e}_{-}^{2}(k) \mathfrak{e}_{-}^{2}\left(k^{\prime}\right)}{R\left(k, k^{\prime}\right)} \preceq \frac{\mathfrak{e}_{-}(k) \mathfrak{e}_{-}\left(k^{\prime}\right)}{\mathfrak{e}_{-}(k)+\mathfrak{e}_{-}\left(k^{\prime}\right)} \preceq 1,
$$

This together with (10.4) imply (10.10). The case $\iota=+$ can be argued similarly. The proof of (10.11) is a consequence of (10.4) and (10.8). 


\section{Identification of the Limit of the Wigner Transform}

Recall that $\left(W_{\epsilon}(\cdot)\right)$ is $*$-weakly sequentially compact in $L^{\infty}\left([0, T], \mathcal{A}^{\prime}\right)$ for any $T>0$. Therefore for any $\epsilon_{n} \rightarrow 0$, as $n \rightarrow+\infty$, we can choose a subsequence, denoted in the same way, such that it $*$-weakly converges to some $W(\cdot) \in L^{\infty}\left([0, T], \mathcal{A}^{\prime}\right)$. In light of (5.10) we have

$$
\sup _{t \geq 0}\|W(t)\|_{\mathcal{A}^{\prime}} \leq K_{0},
$$

with $K_{0}$ the same as in (4.6). Therefore, we can define its Laplace-Fourier transform $w(\lambda, p, k)$ for any $\lambda>0$. Thanks to Theorem 10.2, any limit $w(\lambda, p, k)$ obtained this way will be constant in $k$.

In the present section we show that for any $\lambda>\lambda_{0}$, see (10.2), and $p \in \mathbb{R}$ we have either (see (5.7))

$$
\left(\lambda+\frac{\hat{c} p^{2}}{2}\right) w(\lambda, p)=\bar{W}_{0}(p),
$$

or

$$
\left(\lambda+\hat{c}|p|^{3 / 2}\right) w(\lambda, p)=\bar{W}_{0}(p),
$$

depending on whether the potential is pinning or not. Coefficients $\hat{c}$ are as in Theorems 5.1 and 5.2, respectively. Since the functions given by either (5.19) or (5.21) have the Laplace transforms that satisfy (11.2) and (11.3), respectively, we conclude from the uniqueness of the corresponding Laplace-Fourier transform that $\left(W_{\epsilon}(\cdot)\right)$ is indeed *-weakly convergent, as $\epsilon \rightarrow 0+$, in $L^{\infty}\left([0, T], \mathcal{A}^{\prime}\right)$, for any $T>0$. This would end the proof of the assertions made in Theorems 5.1 and 5.2. The only two facts, which still require proofs are identities (11.2) and (11.3).

11.1. Derivation of (11.2) and (11.3). Recall the definition

$$
w_{\epsilon}^{(\iota)}(\lambda, p):=\int_{\mathbb{T}} \bar{w}_{\epsilon}(\lambda, p, k) \mathfrak{e}_{\iota}(k) d k, \quad \iota \in\{-,+\} .
$$

Let $B_{M}:=[p:|p|<M]$. In light of Theorem 10.2 it suffices only to show that any *-weak limit of $w_{\epsilon}^{(+)}(\lambda, p)$ in $L^{\infty}\left(I \times \bar{B}_{M}\right)$ satisfies either (11.2), or (11.3) depending on whether the potential is pinning or not.

To abbreviate the notation we omit the arguments of the functions appearing in the ensuing calculations. From the first equation of the system (10.5) we get

$$
D^{(\epsilon)} \bar{w}_{\epsilon}=\epsilon^{\delta} \widehat{W}_{\epsilon}^{(0)}+\frac{3}{2} \gamma \sum_{\iota \in\{-,+\}} \mathfrak{e}_{\iota} w_{\epsilon}^{(-\imath)}+q_{\epsilon},
$$

where

$$
D^{(\epsilon)}:=\epsilon^{\delta} \lambda+2 \gamma R_{\epsilon}+i \epsilon \delta_{\epsilon} \omega,
$$

and $q_{\epsilon}:=\sum_{i=1}^{4} q_{\epsilon}^{(i)}$, with

$$
\begin{aligned}
q_{\epsilon}^{(1)} & :=-\frac{\gamma(\pi \epsilon p)^{2}}{2}\left[\mathfrak{f}_{+} \int_{\mathbb{T}} \bar{w}_{\epsilon}\left(4 \mathfrak{f}_{+}+\mathfrak{e}_{-}\right) d k^{\prime}+3 \mathfrak{f}_{-} \int_{\mathbb{T}} \bar{w}_{\epsilon} \mathfrak{e}_{+} d k^{\prime}\right] \\
q_{\epsilon}^{(2)}: & =-\gamma \mathcal{L} \bar{u}_{\epsilon,+}, \quad q_{\epsilon}^{(3)}:=-i \epsilon \gamma R^{\prime} p \bar{u}_{\epsilon,-}, \\
q_{\epsilon}^{(4)} & :=\frac{\gamma(\pi \epsilon p)^{2}}{2}\left(\delta^{2} \mathcal{L}\right) \bar{u}_{\epsilon,+}+\epsilon^{3} \bar{r}_{\epsilon}^{(1)} .
\end{aligned}
$$


In addition, thanks to (10.3) and (10.4), the remainder $\bar{r}_{\epsilon}^{(1)}$ satisfies: for any compact set $I \subset\left(\lambda_{0},+\infty\right)$ and $M>0$

$$
\limsup _{\epsilon \rightarrow 0+} \sup _{\lambda \in I,|p| \leq M}\left\|r_{\epsilon}^{(1)}(\lambda, p)\right\|_{L^{1}(\mathbb{T})}<+\infty .
$$

Computing $\bar{w}_{\epsilon}$ from (11.4) and then multiplying scalarly both sides of the resulting equation by $\gamma \mathfrak{e}_{\iota}, \iota \in\{-,+\}$ we get the following system

$$
\begin{gathered}
\gamma w_{\epsilon}^{(\iota)} \int_{\mathbb{T}}\left(1-\frac{3 \gamma \mathfrak{e}_{-} \mathfrak{e}_{+}}{2 D^{(\epsilon)}}\right) d k-\frac{3 \gamma^{2}}{2} w_{\epsilon}^{(-\iota)} \int_{\mathbb{T}} \frac{\mathfrak{e}_{\iota}^{2}}{D^{(\epsilon)}} d k \\
=\gamma \epsilon^{\delta} \int_{\mathbb{T}} \frac{\mathfrak{e}_{\iota} \widehat{W}_{\epsilon}^{(0)}}{D^{(\epsilon)}} d k+\gamma \int_{\mathbb{T}} \frac{\mathfrak{e}_{\iota} q_{\epsilon}}{D^{(\epsilon)}} d k, \quad \iota \in\{-,+\} .
\end{gathered}
$$

Adding sideways the above equations corresponding to both values of $\iota$ and then dividing both sides of the resulting equation by $\epsilon^{\delta}$ we obtain

$$
a_{w}^{(\epsilon)} w_{\epsilon}^{(+)}-a_{+}^{(\epsilon)}\left(w_{\epsilon}^{(+)}-w_{\epsilon}^{(-)}\right)=\frac{4 \gamma}{3} \int_{\mathbb{T}} \frac{R \widehat{W}_{\epsilon}^{(0)}}{D^{(\epsilon)}} d k+\frac{4 \gamma}{3 \epsilon^{\delta}} \int_{\mathbb{T}} \frac{R q_{\epsilon}}{D^{(\epsilon)}} d k,
$$

where

$$
\begin{aligned}
& a_{w}^{(\epsilon)}(\lambda, p):=\frac{4 \gamma}{3 \epsilon^{\delta}} \int_{\mathbb{T}}\left(1-\frac{2 \gamma R}{D^{(\epsilon)}}\right) R d k, \\
& a_{+}^{(\epsilon)}(\lambda, p):=\frac{\gamma}{\epsilon^{\delta}} \int_{\mathbb{T}}\left(1-\frac{2 \gamma R}{D^{(\epsilon)}}\right) \mathfrak{e}_{+} d k .
\end{aligned}
$$

Let $\theta(\delta)=0$, when $\delta<2$ and $\theta(2)=1$. The following result, obviously implies either (11.2) or (11.3), under an appropriate hypothesis on the respective dispersion relation.

Proposition 11.1. For any $J \in \mathcal{S}$ such that $J(y, k) \equiv J(y)$ and $\lambda>\lambda_{0}$ we have

$$
\lim _{\epsilon \rightarrow 0+}\left(\frac{4 \gamma}{3} \int_{\mathbb{R} \times \mathbb{T}} \frac{R \widehat{W}_{\epsilon}^{(0)}}{D^{(\epsilon)}} \hat{J} d p d k-\frac{2}{3} \int_{\mathbb{R} \times \mathbb{T}} \widehat{W}_{0} \hat{J} d p d k\right)=0 .
$$

In addition, for any $M>0$ and a compact interval $I \subset\left(\lambda_{0},+\infty\right)$

$$
\lim _{\epsilon \rightarrow 0+} \sup _{\lambda \in I,|p| \leq M}\left|a_{+}^{(\epsilon)}(\lambda, p)\left(w_{\epsilon}^{(+)}(\lambda, p)-w_{\epsilon}^{(-)}(\lambda, p)\right)\right|=0 .
$$

Moreover, under the assumptions of Theorem 5.1, we have

$$
\lim _{\epsilon \rightarrow 0+} \sup _{\lambda \in I,|p| \leq M}\left|a_{w}^{(\epsilon)}(\lambda, p)-\frac{2 \lambda}{3}-\frac{\hat{\sigma}^{2} p^{2}}{3 \gamma_{0}}\right|=0
$$

where $\hat{\sigma}$ is given by (3.17), and

$$
\lim _{\epsilon \rightarrow 0+} \sup _{\lambda \in I,|p| \leq M}\left|\frac{4 \gamma}{3 \epsilon^{\delta}} \int_{\mathbb{T}} \frac{R q_{\epsilon}}{D^{(\epsilon)}} d k-\frac{8 \theta(\delta) \gamma_{0}(\pi p)^{2}}{3} \bar{w}_{\epsilon}^{(+)}\right|=0 .
$$


If, on the other hand, the assumptions of Theorem 5.2 hold then

$$
\lim _{\epsilon \rightarrow 0+} \sup _{\lambda \in I,|p| \leq M}\left|a_{w}^{(\epsilon)}(\lambda, p)-\frac{2 \lambda}{3}-\frac{2 \hat{c}|p|^{3 / 2}}{3}\right|=0
$$

with $\hat{c}$ is given by (3.21), and

$$
\lim _{\epsilon \rightarrow 0+} \sup _{\lambda \in I,|p| \leq M}\left|\frac{4 \gamma}{3 \epsilon^{\delta}} \int_{\mathbb{T}} \frac{R q_{\epsilon}}{D^{(\epsilon)}} d k\right|=0 .
$$

Equalities (11.2) and (11.3) then follow directly from the above proposition by taking the limit, as $\epsilon \rightarrow 0+$, in (11.8).

\subsection{Proof of Proposition 11.1.}

Proof of (11.10). It suffices only to prove that, for any $J$ as in the statement of (11.10)

$$
\lim _{\epsilon \rightarrow 0+}\left|\frac{4 \gamma}{3} \int_{\mathbb{R} \times \mathbb{T}} \frac{R \widehat{W}_{\epsilon}^{(0)}}{D^{(\epsilon)}} \hat{J} d p d k-\frac{2}{3} \int_{\mathbb{R} \times \mathbb{T}} \widehat{W}_{\epsilon}^{(0)} \hat{J} d p d k\right|=0 .
$$

Then equality (11.10) is a consequence of (5.6). Note that $2 \gamma R / D^{(\epsilon)}$ is bounded and convergent to 1 , as $\epsilon \rightarrow 0+$. Using Cauchy-Schwarz inequality we can estimate the expression under the limit in (11.16) by

$$
\frac{2}{3}\left\{\int_{\mathbb{R} \times \mathbb{T}}\left|\frac{2 \gamma R}{D^{(\epsilon)}}-1\right|^{2}|\hat{J}|^{2} d p d k\right\}^{1 / 2}\left\{\int_{\mathbb{R} \times \mathbb{T}}\left|\widehat{W}_{\epsilon}^{(0)}\right|^{2} d p d k\right\}^{1 / 2} .
$$

The first integral tends to 0 , as $\epsilon \rightarrow 0+$, by virtue of the Lebesgue dominated convergence theorem, while the second one remains bounded thanks to condition (4.3). Thus (11.10) follows.

Proof of (11.12) and (11.14). From (11.9) we get

$$
a_{w}^{(\epsilon)}=\frac{4 \gamma}{3} \int_{\mathbb{T}} \frac{R}{\left|D^{(\epsilon)}\right|^{2}}\left\{\left(\epsilon^{\delta} \lambda+2 \gamma R_{\epsilon}\right)\left(\lambda+\frac{\gamma \epsilon^{2-\delta}}{4} p^{2} R^{\prime \prime}\right)+\epsilon^{2-\delta}\left(\delta_{\epsilon} \omega\right)^{2}\right\} d k .
$$

Diffusive scaling. Here $\omega(0)>0,\left(\delta_{\epsilon} \omega\right)^{2} \preceq R_{\epsilon}$ (see (14.9)) and, as we recall $\delta=2-s$. Thus (11.12) follows.

Super-diffusive scaling. In this case $\omega(k) \approx|\sin (\pi k)|$ and $\delta=(3-s) / 2$. From (11.17) we get

$$
\lim _{\epsilon \rightarrow 0+} a_{w}^{(\epsilon)}(\lambda, p)=\frac{2 \lambda}{3}+\lim _{\epsilon \rightarrow 0+} \frac{4 \gamma \epsilon^{2-\delta}}{3} \int_{\mathbb{T}} \frac{R\left(\delta_{\epsilon} \omega\right)^{2}}{\left|D^{(\epsilon)}\right|^{2}} d k
$$


We shall show that

$$
\lim _{\epsilon \rightarrow 0+} \frac{4 \gamma \epsilon^{2-\delta}}{3} \int_{\mathbb{T}} \frac{R\left(\delta_{\epsilon} \omega\right)^{2}}{\left|D^{(\epsilon)}\right|^{2}} d k=\frac{2 \hat{c}|p|^{3 / 2}}{3}
$$

uniformly in $\lambda \in I$ and $|p| \leq M$. Here $\hat{c}$ is given by (3.21).

Assume that $p>0$, as the consideration in the case $p<0$ is analogous. Divide the domain of integration in the integral appearing in (11.19) into three sets $|k| \leq \epsilon^{\rho_{1}}$, $\epsilon^{\rho_{1}} \leq|k| \leq \epsilon^{\rho_{2}}$ and $\epsilon^{\rho_{2}} \leq|k|$, with $\rho_{1}>\rho_{2}>0$ to be adjusted later on, and denote the expressions corresponding to the resulting integrals by $I_{1}^{(\epsilon)}, I_{2}^{(\epsilon)}$ and $I_{3}^{(\epsilon)}$.

The limit of $I_{1}^{(\epsilon)}$. Suppose that $\rho_{1} \in(\delta-1,1)$. Since in the unpinned case $\delta \leq 3 / 2$, it is possible to find such $\rho_{1}$. Using the fact that

$$
\left|D^{(\epsilon)}\right|^{2} \succeq \epsilon\left|\delta_{\epsilon} \omega\right|\left(\gamma R_{\epsilon}+\epsilon^{\delta}\right),
$$

we conclude

$$
I_{1}^{(\epsilon)} \preceq \epsilon^{1-\delta} \int_{0}^{\epsilon^{\rho_{1}}} \frac{\gamma k^{2}}{\gamma k^{2}+\epsilon^{\delta}} d k \leq \epsilon^{1+\rho_{1}-\delta} \rightarrow 0, \quad \text { as } \epsilon \rightarrow 0+.
$$

The limit of $I_{2}^{(\epsilon)}$. Suppose also that $\rho_{2} \in(0,2-\delta-s)$. Since $s \in[0,1)$ we have $2-\delta-s>0$ (recall that $\delta=(3-s) / 2)$. We have

$$
\rho_{2}<2-\delta-s=\delta-1<\rho_{1} .
$$

In the integral appearing in $I_{2}^{(\epsilon)}$ we change variable according to

$$
k^{\prime}:=\frac{\gamma_{0}^{1 / 2} k}{C_{*}^{1 / 2} \epsilon^{(1-s) / 2}} \text { and } C_{*}:=\left(\frac{\hat{\alpha}^{\prime \prime}(0)}{2}\right)^{1 / 2} p .
$$

Define $k_{\epsilon}:=C_{*}^{1 / 2} \epsilon^{(1-s) / 2} k \gamma_{0}^{-1 / 2}$,

$$
\tilde{R}(k):=\frac{\gamma_{0}}{\epsilon^{1-s} C_{*}} R\left(k_{\epsilon}\right), \quad \tilde{R}_{\epsilon}(k):=\frac{\gamma_{0}}{\epsilon^{1-s} C_{*}} R_{\epsilon}\left(k_{\epsilon}\right),
$$

and

$$
\widehat{\delta_{\epsilon} \omega}(k, p):=\frac{1}{C_{*}} \delta_{\epsilon} \omega\left(k_{\epsilon}, p\right) .
$$

Then,

$$
I_{2}^{(\epsilon)}=\frac{\left(\hat{\alpha}^{\prime \prime}(0)\right)^{3 / 4} p^{3 / 2}}{2^{3 / 4} \cdot 3 \gamma_{0}^{1 / 2}} \int_{\mathcal{I}_{\epsilon}} \frac{\tilde{R}\left(\widehat{\delta_{\epsilon} \omega}\right)^{2}}{\left|\hat{D}^{(\epsilon)}\right|^{2}} d k
$$

where

$$
\left|\hat{D}^{(\epsilon)}\right|^{2}:=\left(2 \tilde{R}_{\epsilon}(k)+\frac{\epsilon^{\delta-1} \lambda}{C_{*}}\right)^{2}+\left(\widehat{\delta_{\epsilon} \omega}\right)^{2}
$$


and

$$
\mathcal{I}_{\epsilon}:=\left[k: \frac{\gamma_{0}^{1 / 2} \epsilon^{\bar{\rho}_{1}}}{C_{*}^{1 / 2}} \leq|k| \leq \frac{\gamma_{0}^{1 / 2} \epsilon^{\bar{\rho}_{2}}}{C_{*}^{1 / 2}}\right],
$$

with $\bar{\rho}_{i}:=\rho_{i}-(1-s) / 2, i=1,2$. Note that, according to (11.20),

$$
\bar{\rho}_{1}>\delta-1-\frac{1-s}{2}=0
$$

and

$$
\bar{\rho}_{2}<2-\delta-s-\frac{1-s}{2}=0 .
$$

From (3.18) (and (2.7)) we conclude that both $\tilde{R}(k)$ and $\tilde{R}_{\epsilon}(k)$ converge uniformly to $6 \pi^{2} k^{2}$ when $k \in \mathcal{I}_{\epsilon},|p| \leq M$. Likewise, $\widehat{\delta_{\epsilon} \omega}(k, p)$ converges uniformly to 1 when $k \in \mathcal{I}_{\epsilon}$ and $\lambda \in I,|p| \leq M$. Since in addition

$$
\frac{\tilde{R}(k)\left(\widehat{\delta_{\epsilon} \omega}(k)\right)^{2} 1_{\mathcal{I}_{\epsilon}}(k)}{\left|\hat{D}^{(\epsilon)}(k)\right|^{2}} \preceq \frac{k^{2}+1}{k^{4}+1}, \quad k \in \mathbb{R}
$$

we obtain

$$
\lim _{\epsilon \rightarrow 0+} I_{2}^{(\epsilon)}=\frac{\hat{\alpha}^{\prime \prime}(0)^{3 / 4} p^{3 / 2}}{3 \cdot 2^{3 / 4} \gamma_{0}^{1 / 2}} \int_{\mathbb{R}} \frac{24 \pi^{2} k^{2}}{144 \pi^{4} k^{4}+1} d k
$$

and the convergence is uniform in $\lambda \in I$ and $|p| \leq M$. Using the calculus of residua one can show that

$$
\int_{-\infty}^{+\infty} \frac{k^{2}}{k^{4}+1} d k=\frac{\pi}{\sqrt{2}}
$$

therefore

$$
\int_{\mathbb{R}} \frac{24 \pi^{2} k^{2}}{144 \pi^{4} k^{4}+1} d k=\frac{1}{\sqrt{6}} .
$$

Thus,

$$
\lim _{\epsilon \rightarrow 0+} I_{2}^{(\epsilon)}=\frac{\left(\hat{\alpha}^{\prime \prime}(0)\right)^{3 / 4} p^{3 / 2}}{3^{3 / 2} \cdot 2^{5 / 4} \gamma_{0}^{1 / 2}}
$$

The limit of $I_{3}^{(\epsilon)}$. Then,

$$
I_{3}^{(\epsilon)} \preceq \gamma \epsilon^{2-\delta} \int_{|k| \geq \epsilon^{\rho_{2}}} \frac{R\left(\delta_{\epsilon} \omega\right)^{2}}{\left|D^{(\epsilon)}\right|^{2}} d k \preceq \gamma \epsilon^{2-\delta} \int_{|k| \geq \epsilon^{\rho_{2}}} \frac{1}{(\gamma k)^{2}} d k \preceq \epsilon^{2-\delta-s-\rho_{2}} \rightarrow 0,
$$

as $\epsilon \rightarrow 0+$, uniformly in $\lambda \in I$ and $|p| \leq M$ (recall that $\rho_{2} \in(0,2-\delta-s)$ ). It ends the proof of (11.19), thus finishing the proof of (11.14). 
Proof of (11.11). It is a simple consequence of the following.

Lemma 11.2. Under the assumptions of Proposition 11.1 we have

$$
\sup _{\lambda \in I,|p| \leq M}\left|a_{+}^{(\epsilon)}(\lambda, p)\right| \preceq 1, \quad \epsilon \in(0,1] .
$$

Proof. Similarly as in (11.17) we get $a_{+}^{(\epsilon)}=a_{+, 1}^{(\epsilon)}+a_{+, 2}^{(\epsilon)}$, where

$$
\begin{aligned}
& a_{+, 1}^{(\epsilon)}:=\gamma \int_{\mathbb{T}} \frac{\mathfrak{e}_{+}}{\left|D^{(\epsilon)}\right|^{2}}\left(\epsilon^{\delta} \lambda+2 \gamma R_{\epsilon}\right)\left(\lambda+\gamma \epsilon^{2-\delta} \frac{p^{2} R^{\prime \prime}}{4}\right) d k, \\
& a_{+, 2}^{(\epsilon)}:=\epsilon^{2-\delta} \gamma \int_{\mathbb{T}} \frac{\left(\delta_{\epsilon} \omega\right)^{2} \mathfrak{e}_{+}}{\left|D^{(\epsilon)}\right|^{2}} d k
\end{aligned}
$$

Term $\left|a_{+, 1}^{(\epsilon)}\right|$ is bounded, due to the fact that $\gamma \mathfrak{e}_{+}\left(\epsilon^{\delta} \lambda+2 \gamma R_{\epsilon}\right) \preceq\left|D^{(\epsilon)}\right|^{2}$. To bound the term $a_{+, 2}^{(\epsilon)}$ in the pinned case we use the fact that then $\left(\delta_{\epsilon} \omega\right)^{2} \preceq R_{\epsilon}$. In the case $\omega(0)=0$ we use the bound $\mathfrak{e}_{+} \preceq R$. Then, the conclusion of the lemma follows from (11.19).

Proof of (11.13) and (11.15). Denote

$$
Q_{\epsilon}^{(i)}(\lambda, p):=\frac{4 \gamma}{3 \epsilon^{\delta}} \int_{\mathbb{T}} \frac{R q_{\epsilon}^{(i)}}{D^{(\epsilon)}} d k
$$

The equalities in question follow easily from our next result.

Lemma 11.3. Under the assumptions of Proposition 11.1 we have

$$
\lim _{\epsilon \rightarrow 0+} \sup _{\lambda \in I,|p| \leq M}\left|Q_{\epsilon}^{(i)}(\lambda, p)\right|=0, \quad i=2,3,4 .
$$

Equality (11.23) holds also for $Q_{\epsilon}^{(1)}(\lambda, p)$ when $\delta<2$. When $\omega(0)>0$ and $\delta=2$ we have

$$
\lim _{\epsilon \rightarrow 0+} \sup _{\lambda \in I,|p| \leq M}\left|Q_{\epsilon}^{(1)}(\lambda, p)-\frac{8 \gamma_{0}(\pi p)^{2}}{3} w_{\epsilon}^{(+)}(\lambda, p)\right|=0 .
$$

Proof. Since $|\gamma R| /\left|D^{(\epsilon)}\right|$ is bounded the conclusion of the lemma for $i=4$ is a simple consequence of (10.11). When $i=3$, both $k \mapsto \delta_{\epsilon} \omega(k ; p)$ and $k \mapsto \hat{R}^{\prime}(k)$ are odd. Since $k \mapsto \bar{u}_{\epsilon,-}(\lambda, p, k)$ is even, we can write

$$
Q_{\epsilon}^{(3)}(\lambda, p)=-\frac{4 \epsilon^{2-\delta} \gamma^{2}}{3} \int_{\mathbb{T}} \frac{R R^{\prime} p \delta_{\epsilon} \omega \bar{u}_{\epsilon,-}}{\left|D^{(\epsilon)}\right|^{2}} d k .
$$

In case $\omega(0)>0$ we use $\left|R^{\prime} p \delta_{\epsilon} \omega\right| \preceq R_{\epsilon}$ (see (14.9) below), therefore

$$
\left|Q_{\epsilon}^{(3)}(\lambda, p)\right| \preceq \epsilon^{2-\delta} \int_{\mathbb{T}}\left|\bar{u}_{\epsilon,-}\right| d k
$$

and the lemma follows then by virtue of (10.11). 
In the unpinned case we use the bound

$$
\left|D^{(\epsilon)}\right| \succeq \gamma R_{\epsilon}+\epsilon\left|\delta_{\epsilon} \omega\right|
$$

together with Cauchy-Schwartz inequality and (10.8). We obtain

$$
\begin{aligned}
\left|Q_{\epsilon}^{(3)}(\lambda, p)\right| & \leq \frac{4 \epsilon^{2-\delta} \gamma^{2}|p|}{3}\left\{\int_{\mathbb{T}} R\left|\bar{u}_{\epsilon,-}\right|^{2} d k\right\}^{1 / 2}\left\{\int_{\mathbb{T}} \frac{R\left(R^{\prime} \delta_{\epsilon} \omega\right)^{2}}{\left|D^{(\epsilon)}\right|^{4}} d k\right\}^{1 / 2} \\
& \preceq \gamma^{2} \epsilon^{2-\delta} \epsilon^{(\delta-s) / 2}\left\{\int_{\mathbb{T}} \frac{R\left(R^{\prime} \delta_{\epsilon} \omega\right)^{2}}{\left(\gamma R_{\epsilon}\right)^{4}+\epsilon^{4}\left|\delta_{\epsilon} \omega\right|^{4}} d k\right\}^{1 / 2} \\
& \leq \gamma^{2} \epsilon^{2-(\delta+s) / 2}\left\{\int_{\mathbb{T}} \frac{R\left(R^{\prime} \delta_{\epsilon} \omega\right)^{2}}{\left(\gamma R_{\epsilon}\right)^{2} \epsilon^{2}\left|\delta_{\epsilon} \omega\right|^{2}} d k\right\}^{1 / 2} \\
& =\gamma \epsilon^{1-(\delta+s) / 2}\left\{\int_{\mathbb{T}} \frac{R\left(R^{\prime}\right)^{2}}{R_{\epsilon}^{2}} d k\right\}^{1 / 2} \\
& \preceq \epsilon^{(2+s-\delta) / 2}\left\{\int_{0}^{1} \frac{k^{3}}{k^{4}+\epsilon^{4}} d k\right\}^{1 / 2} \\
& \preceq \epsilon^{(2+s-\delta) / 2} \log ^{1 / 2}\left(\frac{1}{\epsilon}\right) \rightarrow 0,
\end{aligned}
$$

as $\epsilon \rightarrow 0+$, uniformly in $\lambda \in I$ and $|p| \leq M$.

Next,

$$
\begin{aligned}
Q_{\epsilon}^{(2)}(\lambda, p)= & -\frac{4 \gamma}{3 \epsilon^{\delta}} \int_{\mathbb{T}} \frac{R \mathcal{L} \bar{u}_{\epsilon,+}}{D^{(\epsilon)}} d k=-\frac{4 \gamma}{3 \epsilon^{\delta}} \int_{\mathbb{T}} \frac{\left(R-R_{\epsilon}\right) \mathcal{L} \bar{u}_{\epsilon,+}}{D^{(\epsilon)}} d k \\
& +\frac{4 \gamma}{3 \epsilon^{\delta}} \int_{\mathbb{T}} \frac{\left(\epsilon^{\delta} \lambda+i \epsilon \delta_{\epsilon} \omega\right) \mathcal{L} \bar{u}_{\epsilon,+}}{D^{(\epsilon)}} d k-\frac{4 \gamma}{3 \epsilon^{\delta}} \int_{\mathbb{T}} \mathcal{L} \bar{u}_{\epsilon,+} d k
\end{aligned}
$$

Denote the terms appearing on the utmost right hand of (11.26) by $I_{\epsilon}, I I_{\epsilon}$ and $I I I_{\epsilon}$, respectively. Since $\int_{\mathbb{T}} \mathcal{L} f d k=0$ for any $f \in L^{1}(\mathbb{T})$ we have $I I I_{\epsilon}=0$. In addition, (see (9.3))

$$
\begin{aligned}
I_{\epsilon} & =\frac{\gamma \epsilon^{\delta-2} p^{2}}{6} \int_{\mathbb{T}} \frac{R^{\prime \prime} \mathcal{L} \bar{u}_{\epsilon,+}}{D^{(\epsilon)}} d k \\
& =\sum_{\iota \in\{-,+\}} \frac{\gamma \epsilon^{\delta-2} p^{2}}{4} u_{\epsilon,+}^{(\iota)} \int_{\mathbb{T}} \frac{R^{\prime \prime} \mathfrak{e}_{-\iota}}{D^{(\epsilon)}} d k-\frac{\gamma \epsilon^{\delta-2} p^{2}}{3} \int_{\mathbb{T}} \frac{R^{\prime \prime} R}{D^{(\epsilon)}} \bar{u}_{\epsilon,+} d k .
\end{aligned}
$$

Here $u_{\epsilon,+}^{(\iota)}(\lambda, p):=\left\langle\bar{u}_{\epsilon,+}(\lambda, p), \mathfrak{e}_{\iota}\right\rangle_{L^{2}(\mathbb{T})}$ for $\iota \in\{-,+\}$. Note that

$$
\sup _{\epsilon \in(0,1]} \sup _{\lambda \in I,|p| \leq M}\left\|\frac{\gamma R^{\prime \prime} R}{D^{(\epsilon)}}\right\|_{L^{\infty}(\mathbb{T})}<+\infty
$$

If $\omega(0)>0$, then, according to (10.8) we have

$$
G_{\epsilon}:=\int_{\mathbb{T}} \frac{R_{\epsilon}\left|\bar{u}_{\epsilon,+}\right|^{2}}{\left(\epsilon^{\delta}+\gamma R_{\epsilon}\right)^{2}} d k \preceq \epsilon^{\delta-s}, \quad \lambda \in I,|p| \leq M, \epsilon \in(0,1] .
$$


Hence, $\left(\right.$ since $\left.\gamma=\epsilon^{s} \gamma_{0}\right)$

$$
\left|u_{\epsilon,+}^{(\iota)}\right| \preceq G_{\epsilon}^{1 / 2}\left\{\int_{\mathbb{T}} R_{\epsilon}\left(\epsilon^{\delta}+\gamma R_{\epsilon}\right)^{2} d k\right\}^{1 / 2} \preceq \epsilon^{(\delta+s) / 2}
$$

and, using the estimate $\left|D_{\epsilon}\right| \geq \epsilon^{\delta}+\gamma R_{\epsilon}(k)$, we get

$$
\int_{\mathbb{T}} \frac{R\left|\bar{u}_{\epsilon,+}\right|}{\left|D^{(\epsilon)}\right|} d k \preceq G_{\epsilon}^{1 / 2} \preceq \epsilon^{(\delta-s) / 2} .
$$

This leads to estimate (recall that $\delta=2-s$ )

$$
\left|I_{\epsilon}\right| \preceq \epsilon^{\delta-2} \epsilon^{(\delta+s) / 2}=\epsilon^{\delta-1} .
$$

In the unpinned case, $\bar{\omega}(k, \epsilon p) \approx R_{\epsilon}(k)$, therefore from (10.8) we get

$$
H_{\epsilon}:=\int_{\mathbb{T}} \frac{R_{\epsilon}^{2}\left|\bar{u}_{\epsilon,+}\right|^{2}}{\left(\epsilon^{\delta}+\gamma R_{\epsilon}\right)^{2}} d k \preceq \epsilon^{\delta-s}, \quad \lambda \in I,|p| \leq M, \epsilon \in(0,1] .
$$

Hence,

$$
\left|u_{\epsilon,+}^{(\iota)}\right| \preceq H_{\epsilon}^{1 / 2}\left\{\int_{\mathbb{T}}\left(\epsilon^{\delta}+\gamma R_{\epsilon}\right)^{2} d k\right\}^{1 / 2} \preceq \epsilon^{(\delta+s) / 2}
$$

and, using again $\left|D_{\epsilon}\right| \geq \epsilon^{\delta}+\gamma R_{\epsilon}(k)$, we obtain

$$
\int_{\mathbb{T}} \frac{R\left|\bar{u}_{\epsilon,+}\right|}{\left|D^{(\epsilon)}\right|} d k \preceq H_{\epsilon}^{1 / 2} \preceq \epsilon^{(\delta-s) / 2} .
$$

This leads to estimate (recall that $\delta=(3-s) / 2)$

$$
\left|I_{\epsilon}\right| \preceq \epsilon^{\delta-2} \epsilon^{(\delta+s) / 2}=\epsilon^{(1-s) / 4} .
$$

We have shown therefore that in both cases $\lim _{\epsilon \rightarrow 0+} I_{\epsilon}=0$.

Concerning term $I I_{\epsilon}$ note that, thanks to the fact that $k \mapsto \delta_{\epsilon} \omega(k, p)$ is odd and $k \mapsto \mathcal{L} \bar{u}_{\epsilon,+}(\lambda, p, k)$ is even we have

$$
\begin{aligned}
I I_{\epsilon}= & \frac{4 \gamma}{3} \int_{\mathbb{T}} \frac{\mathcal{L} \bar{u}_{\epsilon,+}}{\left|D^{(\epsilon)}\right|^{2}}\left[\lambda\left(\epsilon^{\delta} \lambda+2 \gamma R_{\epsilon}\right)+\epsilon^{2-\delta}\left(\delta_{\epsilon} \omega\right)^{2}\right] d k \\
= & 2 \gamma \sum_{\iota \in\{-,+\}} u_{\epsilon,+}^{-\iota} \int_{\mathbb{T}} \frac{\mathfrak{e}_{\iota}}{\left|D^{(\epsilon)}\right|^{2}}\left[\lambda\left(\epsilon^{\delta} \lambda+2 \gamma R_{\epsilon}\right)+\epsilon^{2-\delta}\left(\delta_{\epsilon} \omega\right)^{2}\right] d k \\
& -\frac{8 \gamma}{3} \int_{\mathbb{T}} \frac{R \bar{u}_{\epsilon,+}}{\left|D^{(\epsilon)}\right|^{2}}\left[\lambda\left(\epsilon^{\delta} \lambda+2 \gamma R_{\epsilon}\right)+\epsilon^{2-\delta}\left(\delta_{\epsilon} \omega\right)^{2}\right] d k .
\end{aligned}
$$

We conclude therefore that

$$
\left|I I_{\epsilon}\right| \preceq \gamma \epsilon^{2-\delta} \sum_{\iota \in\{-,+\}}\left|u_{\epsilon,+}^{-\iota}\right| \int_{\mathbb{T}} \frac{\mathfrak{e}_{\iota}\left(\delta_{\epsilon} \omega\right)^{2}}{\left|D^{(\epsilon)}\right|^{2}} d k+\gamma \epsilon^{2-\delta} \int_{\mathbb{T}} \frac{R\left|\bar{u}_{\epsilon,+}\right|\left(\delta_{\epsilon} \omega\right)^{2}}{\left|D^{(\epsilon)}\right|^{2}} d k .
$$

Denote the terms appearing on the right hand side by $I I_{\epsilon}^{(1)}$ and $I I_{\epsilon}^{(2)}$, respectively. 
When $\omega(0)>0$ we use the fact that $\left|\delta_{\epsilon} \omega(k, p)\right| \preceq R_{\epsilon}^{1 / 2}(k)$, see (14.9) below. Therefore, $\gamma^{2} R\left(\delta_{\epsilon} \omega\right)^{2}\left|D^{(\epsilon)}\right|^{-2} \preceq 1$ and from (10.11), we get

$$
\lim _{\epsilon \rightarrow 0+} \sup _{\lambda \in I,|p| \leq M}\left|I I_{\epsilon}\right|=0 .
$$

In the unpinned case, we use (11.31) together with $\left|D^{(\epsilon)}\right|^{2} \succeq \epsilon\left|\delta_{\epsilon} \omega\right|\left(\gamma R_{\epsilon}\right)$ and get

$$
\begin{aligned}
\left|I I_{\epsilon}^{(1)}\right| & \preceq \gamma \epsilon^{2-\delta} \epsilon^{(\delta+s) / 2} \int_{\mathbb{T}} \frac{R\left(\delta_{\epsilon} \omega\right)^{2}}{\left|D^{(\epsilon)}\right|^{2}} d k \\
& \preceq \gamma \epsilon^{2+(s-\delta) / 2} \int_{\mathbb{T}} \frac{R\left(\delta_{\epsilon} \omega\right)^{2}}{\epsilon\left(\gamma R_{\epsilon}\right)\left|\delta_{\epsilon} \omega\right|} d k \preceq \epsilon^{(2+s-\delta) / 2} \rightarrow 0,
\end{aligned}
$$

uniformly in $\lambda \in \mathcal{I}$ and $|p| \leq M$, as $\delta=(3-s) / 2<2+s$ for $s \in[0,1)$.

From the Cauchy-Schwartz inequality together with (11.31) we get

$$
\left|I I_{\epsilon}^{(2)}\right| \preceq \gamma \epsilon^{2-\delta}\left\{\int_{\mathbb{T}} \frac{\left(\delta_{\epsilon} \omega\right)^{4}}{\left|D^{(\epsilon)}\right|^{2}} d k\right\}^{1 / 2}\left\{\int_{\mathbb{T}} \frac{R^{2}\left|\bar{u}_{\epsilon,+}\right|^{2}}{\left|D^{(\epsilon)}\right|^{2}} d k\right\}^{1 / 2} .
$$

We use $\left|D^{(\epsilon)}\right|^{2} \succeq \epsilon\left(\epsilon^{\delta}+\gamma R\right)\left|\delta_{\epsilon} \omega\right|$ to estimate the first integral and $\left|D^{(\epsilon)}\right|^{2} \succeq\left(\epsilon^{\delta}+\gamma R\right)^{2}$ together with (11.30) to bound the second one. Therefore

$$
\sup _{\lambda \in I,|p| \leq M}\left|I I_{\epsilon}^{(2)}\right| \preceq \gamma \epsilon^{2-\delta} \epsilon^{(\delta-s) / 2}\left\{\int_{\mathbb{T}} \frac{\left(\delta_{\epsilon} \omega\right)^{3}}{\epsilon\left(\gamma R_{\epsilon}+\epsilon^{\delta}\right)} d k\right\}^{1 / 2} \preceq \epsilon^{(3+s) / 2-\delta} \rightarrow 0,
$$

as $\epsilon \rightarrow 0+$. This ends the proof of (11.23) for $i=2$.

Concerning $Q_{\epsilon}^{(1)}(\lambda, p)$ we can write

$$
\begin{aligned}
Q_{\epsilon}^{(1)}(\lambda, p):= & -\frac{2 \gamma^{2}(\pi p)^{2} \epsilon^{2-\delta}}{3} \int_{\mathbb{T}} \frac{R}{D^{(\epsilon)}}\left[\mathfrak{f}_{+}\left\langle w_{\epsilon}, 4 \mathfrak{f}_{+}+\mathfrak{e}_{-}\right\rangle_{L^{2}(\mathbb{T})}\right. \\
& \left.+3 \mathfrak{f}_{-}\left\langle w_{\epsilon}, \mathfrak{e}_{+}\right\rangle_{L^{2}(\mathbb{T})}\right] d k .
\end{aligned}
$$

Since $\gamma|R| /\left|D^{(\epsilon)}\right|$ is bounded the conclusion of the lemma follows easily for $\delta<2$. If $\delta=2$, then $\gamma \equiv \gamma_{0}$. We can use the Lebesgue dominated convergence theorem and obtain (11.24).

11.3. The dual dynamics. The equations (8.7) and (8.10) describing the dynamics of the column vector $\widehat{\mathbb{W}}_{\varepsilon}(t, p, k)$ given by

$$
\widehat{\mathbb{W}}_{\varepsilon}^{T}(t, p, k)=\left[\widehat{W}_{\varepsilon,+}(t, p, k), \widehat{Y}_{\varepsilon,+}(t, p, k), \widehat{Y}_{\varepsilon,-}(t, p, k), \widehat{W}_{\varepsilon,-}(t, p, k)\right]
$$

can be written in the form

$$
\frac{d}{d t} \widehat{\mathbb{W}}_{\varepsilon}(t, p, k)=\mathbb{L}_{\epsilon} \widehat{\mathbb{W}}_{\varepsilon}(t, p, k)
$$

where $\mathbb{L}_{\epsilon}$ is some matrix operator. We now define the dual dynamics that runs on test functions. Suppose that

$$
\left(\widehat{\mathbb{J}}^{(\epsilon)}\right)^{T}(t, p, k)=\left[\widehat{J}_{\epsilon}^{w,+}(t), \widehat{J}_{\epsilon}^{y,+}(t), \widehat{J}_{\epsilon}^{y,-}(t), \widehat{J}_{\epsilon}^{w,-}(t)\right],
$$


is the solution of the system dual to (11.35), i.e.

$$
\frac{d}{d t} \widehat{J}^{(\epsilon)}(t)=\mathbb{L}_{\epsilon}^{*} \widehat{J}^{(\epsilon)}(t)
$$

with given initial conditions that are the Fourier transforms of some functions belonging to $\mathcal{S}$. The adjoint matrix $\mathbb{L}_{\epsilon}^{*}$ is given explicitly by

$$
\mathbb{L}_{\epsilon}^{*} \widehat{\mathbb{J}}:=\left[\begin{array}{llll}
L_{w}^{(\epsilon)} & L_{-}^{(\epsilon)} & L_{+}^{(\epsilon)} & 0 \\
L_{-}^{(\epsilon)} & L_{y}^{(\epsilon)} & R_{y}^{(\epsilon)} & L_{+}^{(\epsilon)} \\
L_{-}^{(\epsilon)} & R_{y}^{(\epsilon)} & \bar{L}_{y}^{(\epsilon)} & L_{+}^{(\epsilon)} \\
0 & L_{-}^{(\epsilon)} & L_{+}^{(\epsilon)} & \bar{L}_{w}^{(\epsilon)}
\end{array}\right] \widehat{\mathbb{J},}
$$

with

$$
\begin{aligned}
& L_{w}^{(\epsilon)}:=\epsilon^{-\delta}\left(i \epsilon \delta_{\epsilon} \omega(k, p)+\gamma \mathcal{L}_{\epsilon p}^{*}\right), \quad \bar{L}_{w}^{(\epsilon)}:=\epsilon^{-\delta}\left(-i \epsilon \delta_{\epsilon} \omega(k, p)+\gamma \mathcal{L}_{\epsilon p}^{*}\right), \\
& L_{y}^{(\epsilon)}:=\epsilon^{-\delta}\left[i \bar{\omega}(k, \epsilon p)+\gamma\left(\mathcal{L}_{\epsilon p}^{*}-\mathcal{R}_{\epsilon p}^{*}\right)\right], \quad \bar{L}_{y}^{(\epsilon)}:=\epsilon^{-\delta}\left[-i \bar{\omega}(k, \epsilon p)+\gamma\left(\mathcal{L}_{\epsilon p}^{*}-\mathcal{R}_{\epsilon p}^{*}\right)\right], \\
& L_{ \pm}^{(\epsilon)}:=-2^{-1} \gamma \epsilon^{-\delta}\left(\mathcal{L}_{\epsilon p}^{ \pm}\right)^{*}, \quad R_{y}^{(\epsilon)}:=\epsilon^{-\delta} \gamma \mathcal{R}_{\epsilon p}^{*} .
\end{aligned}
$$

The operators $\mathcal{L}_{p}^{*},\left(\mathcal{L}_{p}^{ \pm}\right)^{*}$ and $\mathcal{R}_{p}^{*}$ are the adjoints of $\mathcal{L}_{p}, \mathcal{L}_{p}^{ \pm}$and $\mathcal{R}_{p}^{*}$ (see (8.6) and (8.8)) with respect to the Lebesgue measure on $\mathbb{T}$. Given $M>0$ we introduce the norm

$$
\|J\|_{\mathcal{A}_{2, M}}:=\int_{B_{M}} d p\left\{\int_{\mathbb{T}}|\hat{J}(p, k)|^{2} d k\right\}^{1 / 2},
$$

and denote by $\mathcal{A}_{2, M}$ the completion of $\mathcal{S}$ under the norm and by $\mathcal{A}_{2, M}^{\prime}$ its dual, that is the space of all $J: \mathbb{R} \times \mathbb{T} \rightarrow \mathbb{C}$ equipped with the norm

$$
\|J\|_{\mathcal{A}_{2, M}^{\prime}}:=\sup _{|p| \leq M}\left\{\int_{\mathbb{T}}|\hat{J}(p, k)|^{2} d k\right\}^{1 / 2} .
$$

Let $\mathcal{A}_{2, \text { loc }}:=\bigcap_{M>0} \mathcal{A}_{2, M}$. Given $J \in \mathcal{S}$, define

$$
\underline{\mathrm{J}}(p):=\int_{\mathbb{T}} \hat{J}(p, k) d k=\int_{\mathbb{R} \times \mathbb{T}} e^{-2 \pi i p y} J(y, k) d y d k
$$

and

$$
\varphi(p):= \begin{cases}\frac{\hat{c} p^{2}}{2}, & \text { in case } \hat{\alpha}(0)>0, \\ \hat{c}|p|^{3 / 2}, & \text { in case } \hat{\alpha}(0)=0 .\end{cases}
$$

Coefficient $\hat{c}$ is determined either as in Theorem 5.1, when $\hat{\alpha}(0)>0$, or Theorem 5.2, when $\hat{\alpha}(0)=0$. We can repeat the argument made so far and conclude the following statement concerning the convergence of the dual dynamics.

Proposition 11.4. Suppose that the initial data in (11.36) belongs to $\mathcal{A}_{2, \text { loc }}$. Then the following are true: 
(i) for any $T, M>0$ we have

$$
\sup _{\epsilon \in(0,1]} \sup _{t \in[0, T]}\left\|\mathbb{J}^{(\epsilon)}(t)\right\|_{\mathcal{A}_{2, M}^{\prime}}<+\infty,
$$

(ii) suppose that $M>0$ is such that $\widehat{\mathbb{J}}^{(\epsilon)}(0, p, k) \equiv 0$ for $|p| \geq M$ and $k \in \mathbb{T}$. Then $\widehat{\mathbb{J}}^{(\epsilon)}(t, p, k) \equiv 0$ for $t \geq 0,|p| \geq M$ and $k \in \mathbb{T}$,

(iii) for any $T>0$ and $g \in L^{1}\left([0, T], \mathcal{A}_{2, l o c}\right)$ and $\widehat{\mathbb{J}}^{(\epsilon)}(t, p, k)$ as in ii) we have

$$
\begin{gathered}
\lim _{\epsilon \rightarrow 0} \int_{0}^{T} \int_{B_{M} \times \mathbb{T}} \hat{J}_{\epsilon}^{w,+}(t, p, k) \hat{g}^{*}(t, p, k) d t d p d k \\
=\int_{0}^{T} \int_{\mathbb{R}} e^{-\varphi(p) t} \underline{J}^{w,+}(p) g^{*}(t, p) d t d p .
\end{gathered}
$$

\section{Proofs of Theorems 6.1 and 6.2}

12.1. Evolution of the random Wigner transform. To describe the evolution of the fluctuating Wigner transform $\widetilde{\mathcal{W}}_{\epsilon}(t ; J)$, see $(6.1)$, we shall also need the following quantities

$$
\begin{aligned}
\widetilde{\mathcal{Y}}_{\epsilon,+}(t ; J) & :=\sqrt{\epsilon} \sum_{x, x^{\prime} \in \mathbb{Z}} \psi_{x^{\prime}}^{(\epsilon)}(t) \psi_{x}^{(\epsilon)}(t) \tilde{J}^{*}\left(\frac{\epsilon}{2}\left(x+x^{\prime}\right), x^{\prime}-x\right), \\
\widetilde{\mathcal{Y}}_{\epsilon,-}(t ; J) & :=\sqrt{\epsilon} \sum_{x, x^{\prime} \in \mathbb{Z}}\left(\psi_{x^{\prime}}^{(\epsilon)}(t) \psi_{x}^{(\epsilon)}(t)\right)^{*} \tilde{J}^{*}\left(-\frac{\epsilon}{2}\left(x+x^{\prime}\right), x^{\prime}-x\right), \\
\widetilde{\mathcal{W}}_{\epsilon,-}(t ; J) & :=\sqrt{\epsilon} \sum_{x, x^{\prime} \in \mathbb{Z}}\left(\psi_{x^{\prime}}^{(\epsilon)}(t)\left(\psi_{x}^{(\epsilon)}(t)\right)^{*}-\delta_{x, x^{\prime}} \mathcal{E}_{0}\right) \tilde{J}^{*}\left(-\frac{\epsilon}{2}\left(x+x^{\prime}\right), x^{\prime}-x\right) .
\end{aligned}
$$

We identify $\widetilde{\mathcal{W}}_{\epsilon,+}(t)=\widetilde{\mathcal{W}}_{\epsilon}(t)$. Given the column vector $\mathbb{J}^{T}=\left[J^{w,+}, J^{y,+}, J^{y,-}, J^{w,-}\right]$ with the components that are the Fourier transforms of functions from $\mathcal{S}$, denote

$$
\widetilde{\mathbb{W}}_{\epsilon}(t ; \mathbb{J}):=\sum_{\iota \in\{-,+\}}\left(\widetilde{\mathcal{W}}_{\epsilon, l}\left(t ; J^{w, \iota}\right)+\widetilde{\mathcal{Y}}_{\epsilon, \iota}\left(t ; J^{y, \iota}\right)\right) .
$$

For $J_{1}, J_{2} \in \mathcal{S}$ let

$$
\begin{aligned}
& C_{\epsilon}^{w, \pm}\left(t ; J_{1}, J_{2}\right):=\mathbb{E}\left[\widetilde{\mathcal{W}}_{\epsilon, \pm}\left(t ; J_{1}\right) \widetilde{\mathcal{W}}_{\epsilon}\left(0 ; J_{2}\right)\right], \\
& C_{\epsilon}^{y, \pm}\left(t ; J_{1}, J_{2}\right):=\mathbb{E}\left[\widetilde{\mathcal{Y}}_{\epsilon, \pm}\left(t ; J_{1}\right) \widetilde{\mathcal{W}}_{\epsilon}\left(0 ; J_{2}\right)\right] .
\end{aligned}
$$

Computing the time differential as in Sect. 8, we obtain

$$
\begin{aligned}
d \widetilde{\mathcal{W}}_{\epsilon}(t ; J)= & \epsilon^{-\delta}\left\{\widetilde{\mathcal{W}}_{\epsilon}\left(t ;\left(i \epsilon A+\gamma \mathcal{L}_{\epsilon}^{*}\right) J\right)-\frac{\gamma}{2} \sum_{\sigma \in\{-,+\}} \widetilde{\mathcal{Y}}_{\epsilon, \sigma}\left(t ; \mathcal{L}_{\epsilon,-\sigma}^{*} J\right)\right. \\
& \left.+\epsilon^{-1 / 2} \mathcal{E}_{0} \sum_{n \in \mathbb{Z}} \int_{\mathbb{T}}\left(i \epsilon A \hat{J}+\gamma \mathcal{L}_{\epsilon}^{*} \hat{J}\right)\left(\frac{n}{\epsilon}, k\right) d k\right\} d t+d \mathcal{M}_{t}^{(\epsilon)}(J), \quad(12.2) \\
d \widetilde{\mathcal{Y}}_{\epsilon}(t ; J)= & \epsilon^{-\delta}\left\{\widetilde{\mathcal{Y}}_{\epsilon}\left(t ;\left(i B+\gamma \mathcal{L}_{\epsilon}^{*}\right) J\right)+\gamma \widetilde{\mathcal{Y}}_{\epsilon,-}\left(t ; \mathcal{R}_{\epsilon}^{*} J\right)-\gamma \widetilde{\mathcal{Y}}_{\epsilon}\left(t ; \mathcal{R}_{\epsilon}^{*} J\right)\right. \\
& \left.-\frac{\gamma}{2} \sum_{\sigma \in\{-,+\}} \widetilde{\mathcal{W}}_{\epsilon, \sigma}\left(t ; \mathcal{L}_{\epsilon,-\sigma}^{*} J\right)-\frac{\gamma \mathcal{E}_{0}}{2 \epsilon^{1 / 2}} \sum_{n \in \mathbb{Z}} \int_{\mathbb{T}}\left(\mathcal{L}_{\epsilon,-\sigma}^{*} \hat{J}\right)\left(\frac{n}{\epsilon}, k\right) d k\right\} d t \\
& +d \mathcal{N}_{t}^{(\epsilon)}(J),
\end{aligned}
$$


where $\mathcal{M}_{t}^{(\epsilon)}(J), \mathcal{N}_{t}^{(\epsilon)}(J)$, are some square integrable, continuous trajectory martingales. Summarizing, if the test functions $J^{w, \pm}$ and $J^{y, \pm}$ are such that their respective Fourier transforms in the $x$ variable $\hat{J}^{w, \pm}$ and $\hat{J}^{y, \pm}$ belong to $C_{c}^{\infty}(\mathbb{R} \times \mathbb{T})$, then, using (12.2) and (12.3), we obtain

$$
\frac{d}{d t} \mathbb{E}\left[\widetilde{\mathbb{W}_{\epsilon}}(t ; \mathbb{J}) \widetilde{\mathcal{W}}_{\epsilon}(0 ; J)\right]=\mathbb{E}\left[\widetilde{\mathbb{W}_{\epsilon}}\left(t ; \mathbb{L}_{\epsilon}^{*} \mathbb{J}\right) \widetilde{\mathcal{W}}_{\epsilon}(0 ; J)\right]
$$

where $\mathbb{L}_{\epsilon}^{*}$ is given by (11.37).

Suppose that $\mathbb{J}^{(\epsilon)}(t)$ is the solution of the Eq. (11.36). From part (ii) of Proposition 11.4 we conclude that

$$
\widehat{\mathbb{J}}^{(\epsilon)}(t, p, k) \equiv 0, \quad \forall t \geq 0,|p| \geq M, k \in \mathbb{T},
$$

provided that $M>0$ is such that $\widehat{\mathbb{J}}^{(\epsilon)}(0, p, k) \equiv 0$ for all $|p| \geq M, k \in \mathbb{T}$. Combining (12.4) with (11.36) we obtain

$$
\begin{aligned}
\frac{d}{d s} \mathbb{E}\left[\widetilde{\mathbb{W}}_{\epsilon}\left(s ; \mathbb{J}^{(\epsilon)}(t-s)\right) \widetilde{\mathcal{W}}_{\epsilon}(0 ; J)\right]= & \mathbb{E}\left[\widetilde{\mathbb{W}}_{\epsilon}\left(s ; \mathbb{L}_{\epsilon}^{*} \mathbb{J}^{(\epsilon)}(t-s)\right) \widetilde{\mathcal{W}}_{\epsilon}(0 ; J)\right] \\
& +\mathbb{E}\left[\widetilde{\mathbb{W}}_{\epsilon}\left(s ; \frac{d}{d s} \mathbb{J}^{(\epsilon)}(t-s)\right) \widetilde{\mathcal{W}}_{\epsilon}(0 ; J)\right] \equiv 0
\end{aligned}
$$

for all $s \geq 0$. Comparing the values of $\mathbb{E}\left[\widetilde{\mathbb{W}}_{\epsilon}\left(s ; \mathbb{J}^{(\epsilon)}(t-s)\right) \widetilde{\mathcal{W}}_{\epsilon}(0 ; J)\right]$ for $s=t$ and $s=0$ we get

$$
\mathbb{E}\left[\widetilde{\mathbb{W}}_{\epsilon}(t ; \mathbb{J}) \widetilde{\mathcal{W}}_{\epsilon}(0 ; J)\right]=\mathbb{E}\left[\widetilde{\mathbb{W}}_{\epsilon}\left(0 ; \mathbb{J}^{(\epsilon)}(t)\right) \widetilde{\mathcal{W}}_{\epsilon}(0 ; J)\right]
$$

where

$$
\mathbb{J}:=\mathbb{J}^{(\epsilon)}(0)=\left[J^{w,+}, J^{y,+}, J^{y,-}, J^{w,-}\right]^{T} .
$$

Suppose that the initial data satisfies the hypothesis of part (ii) of Proposition 11.4 and that $\hat{J}$ is compactly supported. According to (6.3) the right hand side of (12.6) equals

$$
\begin{aligned}
\mathbb{E}\left[\widetilde{\mathcal{W}}_{\epsilon}\left(0 ; J_{\epsilon}^{w,+}(t)\right) \widetilde{\mathcal{W}}_{\epsilon}(0 ; J)\right] & =\mathcal{E}_{0}^{2} \epsilon \sum_{x} \int_{\mathbb{T}} J_{\epsilon}^{w,+}(t, \epsilon x, k) J^{*}(\epsilon x, k) d k \\
& =\mathcal{E}_{0}^{2} \sum_{n} \int_{\mathbb{R} \times \mathbb{T}} \hat{J}_{\epsilon}^{w,+}\left(t, \frac{n}{\epsilon}-p, k\right) \hat{J}^{*}(-p, k) d p d k
\end{aligned}
$$

The last equality holds, thanks to the Poisson summation formula, see [14], formula (50) on p. 566. Since the supports of $\hat{J}_{\epsilon}^{w,+}(t)$ and $\hat{J}$ are both compact in $p$ for a sufficiently small $\epsilon$ we can write that the right hand side equals

$$
\mathcal{E}_{0}^{2} \int_{\mathbb{R} \times \mathbb{T}} \hat{J}_{\epsilon}^{w,+}(t, p, k) \hat{J}^{*}(p, k) d p d k .
$$

Using (11.40) we conclude that for any compactly supported $\phi \in L^{1}[0,+\infty)$ we have

$$
\begin{aligned}
& \lim _{\epsilon \rightarrow 0+} \int_{0}^{+\infty} \phi(t) \mathbb{E}\left[\widetilde{\mathcal{W}}_{\epsilon}\left(t ; J^{w,+}\right) \widetilde{\mathcal{W}}_{\epsilon}(0 ; J)\right] d t \\
& =\lim _{\epsilon \rightarrow 0+} \int_{0}^{+\infty} \phi(t) \mathbb{E}\left[\widetilde{\mathcal{W}}_{\epsilon}\left(0 ; J_{\epsilon}^{w,+}(t)\right) \widetilde{\mathcal{W}}_{\epsilon}(0 ; J)\right] d t \\
& =\mathcal{E}_{0}^{2} \int_{0}^{+\infty} d t \int_{\mathbb{R}} \phi(t) \exp \left\{-\frac{\hat{c} p^{2} t}{2}\right\} \underline{\mathbf{J}}^{w,+}(p) \underline{\mathbf{J}}(p) d p
\end{aligned}
$$


with $\hat{c}$ given by (3.21) when $\delta<2$, or (3.20) when $\delta=2$ in the case of a pinning potential, or

$$
\begin{aligned}
& \lim _{\epsilon \rightarrow 0+} \int_{0}^{+\infty} \phi(t) \mathbb{E}\left[\widetilde{\mathcal{W}}_{\epsilon}\left(t ; J^{w,+}\right) \widetilde{\mathcal{W}}_{\epsilon}(0 ; J)\right] d t \\
& \quad=\mathcal{E}_{0}^{2} \int_{0}^{+\infty} d t \int_{\mathbb{R}} \phi(t) \exp \left\{-\hat{c}|p|^{3 / 2} t\right\} \underline{\mathbf{J}}^{w,+}(p) \underline{\mathrm{J}}(p) d p
\end{aligned}
$$

with $\hat{c}$ given by (5.22) in the unpinned case. Generalization to arbitrary $J^{w,+}, J \in \mathcal{S}$ and $\phi \in L^{1}[0,+\infty)$ is standard and can be done via an approximation, due to the fact that process $\left(\widetilde{\mathcal{W}}_{\epsilon}(t ; J)\right)_{t \geq 0}$ is stationary.

Remark. Observe that the proof does not really use time stationarity of the initial distribution, in fact it follows that for any initial homogeneous distribution with energy density given by some $\mathcal{E}(k)$ such that $\int_{\mathbb{T}} \mathcal{E}(k) d k=2 \mathcal{E}_{0}$, we have the same result. On the other hand, we do use the stationarity in order to prove the equivalence of the energy distribution (6.11), see Sect. 13.

\section{Equivalence of Energy Functionals}

\subsection{Proof of Proposition 5.3.}

13.1.1. The case of a pinned potential. The left hand side of (5.24) equals $\lim _{\epsilon \rightarrow 0+}\left(I_{\epsilon}+\right.$ $\left.I I_{\epsilon}\right)$, where

$$
\begin{aligned}
I_{\epsilon} & :=\frac{\epsilon}{4} \sum_{x} J(\epsilon x) \mathbb{E}_{\epsilon}\left(\hat{\alpha}(0)\left[\mathfrak{q}_{x}^{(\epsilon)}(t)\right]^{2}-\sum_{y} \alpha_{x-y}\left[\mathfrak{q}_{y}^{(\epsilon)}(t)\right]^{2}\right) \\
& =\frac{\epsilon}{4} \sum_{x} \mathbb{E}_{\epsilon}\left[\mathfrak{q}_{x}^{(\epsilon)}(t)\right]^{2} \int_{\mathbb{R}} e^{2 \pi i \epsilon x p} \hat{J}(p)[\hat{\alpha}(0)-\hat{\alpha}(-\epsilon p)] d p
\end{aligned}
$$

and

$$
\begin{aligned}
I I_{\epsilon} & :=\frac{\epsilon}{2} \sum_{x} J(\epsilon x) \mathbb{E}_{\epsilon}\left[\mathfrak{q}_{x}^{(\epsilon)}(t)\left(\alpha * \mathfrak{q}^{(\epsilon)}(t)\right)_{x}-\left(\tilde{\omega} * \mathfrak{q}^{(\epsilon)}(t)\right)_{x}^{2}\right] \\
& =\frac{\epsilon}{2} \sum_{y, y^{\prime}} \mathbb{E}_{\epsilon}\left(\mathfrak{q}_{y}^{(\epsilon)}(t) \mathfrak{q}_{y^{\prime}}^{(\epsilon)}(t)\right) \sum_{x} \int_{\mathbb{R}} e^{2 \pi i \epsilon x p} \hat{J}(p)\left[\tilde{\omega}_{x-y}(0)-\tilde{\omega}_{x-y}(-\epsilon p)\right] \tilde{\omega}_{x-y^{\prime}} d p .
\end{aligned}
$$

Here we have adopted the notation $\tilde{\omega}_{x}(p):=e^{-2 \pi i x p} \tilde{\omega}_{x}$. Since in the case of a pinned chain we have

$$
\sup _{\epsilon \in(0,1]} \epsilon \mathbb{E}_{\epsilon}\left[\mathfrak{q}_{x}^{(\epsilon)}(t)\right]^{2}<+\infty
$$

it is clear that for any $J \in C_{0}^{\infty}(\mathbb{R})$ we have

$$
\lim _{\epsilon \rightarrow 0+} I_{\epsilon}=0 \text { and } \lim _{\epsilon \rightarrow 0+} I I_{\epsilon}=0 .
$$


13.1.2. The case of an unpinned potential. Then $\hat{\alpha}(0)=0$. Let $\left(\mu_{\epsilon}\right)_{\epsilon \in(0,1]}$, be the family of probability distributions on $\ell^{2}$ such that condition (4.3) holds. We claim that it suffices to prove (5.24) only for $t=0$. Indeed, our argument, presented below, shows that the equivalence of energy density functionals is a consequence of the aforementioned bound (4.3). By virtue of the estimate (9.18) for $p=0$ this bound persists in time, so our proof shows that in fact (5.24) holds for any subsequent time $t \geq 0$. For the purpose of this proof we let

$$
\mathfrak{q}_{x}:=\int_{\mathbb{T}}\left(e^{2 \pi i k x}-1\right) \hat{\mathfrak{q}}(k) d k,
$$

where

$$
\hat{\mathfrak{q}}(k):=\frac{\hat{\psi}(k)+\hat{\psi}^{*}(-k)}{2 \omega(k)} .
$$

As a consequence of (4.6) and (4.3) we obtain

$$
\limsup _{\epsilon \rightarrow 0+} \epsilon\left\langle\sum_{x}(\tilde{\omega} * \mathfrak{q})_{x}^{2}\right\rangle_{\mu_{\epsilon}}<+\infty
$$

and

$$
\limsup _{\epsilon \rightarrow 0+} \int_{\mathbb{T}}\left[\epsilon\left\langle|\omega(k) \hat{\mathfrak{q}}(k)|^{2}\right\rangle_{\mu_{\epsilon}}\right]^{2} d k<+\infty .
$$

Define

$$
\delta \hat{\alpha}\left(k, k^{\prime}\right):=\hat{\alpha}\left(k+k^{\prime}\right)-\hat{\alpha}(k)-\hat{\alpha}\left(k^{\prime}\right) .
$$

Lemma 13.1. We have

$$
\left|\frac{\delta \hat{\alpha}\left(k, k^{\prime}\right)}{\omega(k) \omega\left(k^{\prime}\right)}\right| \preceq 1, \quad k, k^{\prime} \in \mathbb{T} .
$$

Proof. Observe that

$$
\hat{\alpha}(k)=-2 \sum_{x} \alpha_{x} \mathfrak{s}^{2}(x k)
$$

Therefore (13.6) is a consequence of the following elementary inequality

$$
\left|\mathfrak{s}^{2}(\alpha+\beta)-\mathfrak{s}^{2}(\alpha)-\mathfrak{s}^{2}(\beta)\right| \preceq|\mathfrak{s}(\alpha) \mathfrak{s}(\beta)|, \quad \alpha, \beta \in \mathbb{R}
$$

and the assumption (a1) made on the decay of $\left(\alpha_{x}\right)$.

Let

$$
\phi_{x}:=\left|\psi_{x}\right|^{2}-2 \mathfrak{e}_{x}=\frac{1}{2} \sum_{y} \alpha_{x-y}\left(\mathfrak{q}_{x}-\mathfrak{q}_{y}\right)^{2}+(\tilde{\omega} * \mathfrak{q})_{x}^{2}
$$

Obviously Proposition 5.3 is a consequence of the following.

Lemma 13.2. For any $J \in C_{0}^{\infty}(\mathbb{R})$

$$
\lim _{\epsilon \rightarrow 0} \epsilon \sum_{x} J(\epsilon x)\left\langle\phi_{x}\right\rangle_{\mu_{\epsilon}}=0 .
$$

Proof. Using (13.2) we can write $\phi_{x}$ in the Fourier transform coordinates as

$$
\phi_{x}=\iint_{\mathbb{T}^{2}} \hat{F}\left(k, k^{\prime}\right) \omega(k) \hat{\mathfrak{q}}(k) \omega\left(k^{\prime}\right) \hat{\mathfrak{q}}\left(k^{\prime}\right) e^{i 2 \pi\left(k+k^{\prime}\right) x} d k d k^{\prime}
$$


where

$$
\hat{F}\left(k, k^{\prime}\right):=\frac{\hat{\alpha}\left(k+k^{\prime}\right)-\hat{\alpha}(k)-\hat{\alpha}\left(k^{\prime}\right)}{2 \omega(k) \omega\left(k^{\prime}\right)}+1 .
$$

Note that $F(k,-k)=0$. Moreover, according to Lemma 13.1 it is bounded. Observe that under the condition (13.3), function $\omega(k) \hat{\mathfrak{q}}(k)$ is square integrable on $\mathbb{T}$ (although $\hat{\mathfrak{q}}(k)$ need not be so). Furthermore,

$$
\epsilon \sum_{x} J(\epsilon x)\left\langle\phi_{x}\right\rangle_{\mu_{\epsilon}}=\int_{\mathbb{R}} \hat{J}(p) Z_{\epsilon}(p) d p,
$$

where

$$
Z_{\epsilon}(p):=\epsilon \int_{\mathbb{T}} \hat{F}(k,-k-\epsilon p)\left\langle\left.\omega(k) \hat{\mathfrak{q}}(k) \omega(-k-\epsilon p) \hat{\mathfrak{q}}(-k-\epsilon p)\right|_{\mu_{\epsilon}} d p d k .\right.
$$

By the Schwarz inequality and symmetry in $k$ and $k+\epsilon p$, we obtain

$$
\begin{aligned}
\left|Z_{\epsilon}(p)\right| & \leq \int_{\mathbb{T}} d k|\hat{F}(k,-k-\epsilon p)| \epsilon\left\langle\omega(k)^{2}|\hat{\mathfrak{q}}(k)|^{2}\right\rangle_{\mu_{\epsilon}} \\
& \leq\left(\int_{\mathbb{T}} d k|\hat{F}(k,-k-\epsilon p)|^{2}\right)^{1 / 2}\left(\int_{\mathbb{T}} d k\left[\epsilon\left\langle\omega(k)^{2}|\hat{\mathfrak{q}}(k)|^{2}\right\rangle_{\mu_{\epsilon}}\right]^{2}\right)^{1 / 2} \\
& \leq C\left(\int_{\mathbb{T}} d k|\hat{F}(k,-k-\epsilon p)|^{2}\right)^{1 / 2} \underset{\epsilon \rightarrow 0+}{\longrightarrow} 0 .
\end{aligned}
$$

Since $Z_{\epsilon}(p)$ is bounded, the result follows upon an application of the Lebesgue dominated convergence theorem.

13.2. Proof of Proposition 6.3. Obviously, stationarity implies that the limit in (6.11) does not depend on $t$ therefore it suffices to prove (6.11) for $t=0$. For that purpose it is enough to show that

$$
\lim _{\epsilon \rightarrow 0+} \epsilon \mathbb{E}\left[\sum_{x} \phi_{x} J(\epsilon x)\right]^{2}=0
$$

where $\phi_{x}=\sum_{i=1}^{3} \phi_{x}^{(i)}$ and

$$
\begin{aligned}
\phi_{x}^{(1)} & :=\frac{1}{2} \sum_{x^{\prime}} \alpha_{x-x^{\prime}}\left(\mathfrak{q}_{x}-\mathfrak{q}_{x^{\prime}}\right)^{2}, \\
\phi_{x}^{(2)} & :=(\tilde{\omega} * \mathfrak{q})_{x}^{2}, \quad \phi_{x}^{(3)}:=-\hat{\alpha}(0) \mathfrak{q}_{x}^{2} .
\end{aligned}
$$

13.2.1. The case of an unpinned chain. We assume that $\hat{\alpha}(0)=0$, therefore $\phi_{x}^{(3)}=0$. Then, the field $\left(\mathfrak{q}_{x}\right)$ is Gaussian given by

$$
\mathfrak{q}_{x}=\sqrt{\mathcal{E}_{0}} \int_{\mathbb{T}} \frac{e^{2 \pi i k x}-1}{\omega(k)} \hat{w}(d k),
$$


where $\hat{w}(d k)$ is a complex even, Gaussian white noise in $L^{2}(\mathbb{T})$, i.e.

$$
\mathbb{E}\left[\hat{w}(d k) \hat{w}^{*}\left(d k^{\prime}\right)\right]=\delta\left(k-k^{\prime}\right) d k d k^{\prime}, \quad \hat{w}^{*}(d k)=\hat{w}(-d k) .
$$

As a result

$$
\mathbb{E}\left(\mathfrak{q}_{x} \mathfrak{q}_{x^{\prime}}\right)=\mathcal{E}_{0} \int_{\mathbb{T}}\left[e^{2 \pi i k x}-1\right]\left[e^{2 \pi i k x^{\prime}}-1\right]^{*} \alpha^{-1}(k) d k
$$

Note that $\mathbb{E} \phi_{x}=0$ for all $x \in \mathbb{Z}$. Indeed, we have

$$
\begin{aligned}
\mathbb{E} \phi_{x}^{(1)} & =\frac{1}{2} \sum_{x^{\prime}} \alpha_{x-x^{\prime}} \mathbb{E}\left(\mathfrak{q}_{x}-\mathfrak{q}_{x^{\prime}}\right)^{2}=\frac{1}{2} \sum_{x^{\prime}} \alpha_{x^{\prime}} \mathbb{E} \mathfrak{q}_{x^{\prime}}^{2} \\
& =\frac{\mathcal{E}_{0}}{2} \sum_{x^{\prime}} \alpha_{x^{\prime}} \int_{\mathbb{T}}\left[2-\left(e^{2 \pi i k x^{\prime}}+e^{-2 \pi i k x^{\prime}}\right)\right] \alpha^{-1}(k) d k=-\mathcal{E}_{0} \int_{\mathbb{T}} \frac{\alpha(k)}{\alpha(k)} d k=-\mathcal{E}_{0} .
\end{aligned}
$$

In addition, thanks to (13.13),

$$
\begin{aligned}
\mathbb{E} \phi_{x}^{(2)} & =\sum_{x^{\prime}, x^{\prime \prime}} \tilde{\omega}_{x-x^{\prime}} \tilde{\omega}_{x-x^{\prime \prime}} \mathbb{E}\left(\mathfrak{q}_{x^{\prime}} \mathfrak{q}_{x^{\prime \prime}}\right) \\
& =\mathcal{E}_{0} \int_{\mathbb{T}}\left(\sum_{x^{\prime}, x^{\prime \prime}} \tilde{\omega}_{x-x^{\prime}} \tilde{\omega}_{x-x^{\prime \prime}} e^{2 \pi i k\left(x^{\prime}-x^{\prime \prime}\right)}\right) \alpha^{-1}(k) d k=\mathcal{E}_{0} \int_{\mathbb{T}} \omega^{2}(k) \alpha^{-1}(k) d k=\mathcal{E}_{0} .
\end{aligned}
$$

In our next step we calculate

$$
r_{x}:=\mathbb{E}\left(\phi_{x} \phi_{0}\right)=\sum_{i, i^{\prime}=1}^{2} r_{x}^{\left(i, i^{\prime}\right)},
$$

where $r_{x}^{\left(i, i^{\prime}\right)}:=\mathbb{E}\left(\tilde{\phi}_{x}^{(i)} \tilde{\phi}_{0}^{\left(i^{\prime}\right)}\right)$ and $\tilde{\phi}_{x}^{(1)}:=\phi_{x}^{(1)}+\mathcal{E}_{0}$, and $\phi_{x}^{(2)}:=\phi_{x}^{(2)}-\mathcal{E}_{0}$. We have

$$
\begin{aligned}
r_{x}^{(1,1)} & =\frac{1}{2} \sum_{x^{\prime}, x^{\prime \prime}} \alpha_{x-x^{\prime}} \alpha_{x^{\prime \prime}}\left\{\mathbb{E}\left[\left(\mathfrak{q}_{x}-\mathfrak{q}_{x^{\prime}}\right) \mathfrak{q}_{x^{\prime \prime}}\right]\right\}^{2} \\
& =\frac{\mathcal{E}_{0}^{2}}{2} \int_{\mathbb{T}^{2}}\left[\delta \hat{\alpha}\left(k, k^{\prime}\right)\right]^{2} \frac{e^{2 \pi i\left(k+k^{\prime}\right) x}}{\hat{\alpha}(k) \hat{\alpha}\left(k^{\prime}\right)} d k d k^{\prime}, \\
r_{x}^{(2,1)}=r_{x}^{(1,2)} & =\sum_{x^{\prime}} \alpha_{x-x^{\prime}}\left\{\mathbb{E}\left[\left(\mathfrak{q}_{x}-\mathfrak{q}_{x^{\prime}}\right)(\tilde{\omega} * \mathfrak{q})_{0}\right]\right\}^{2} \\
& =\mathcal{E}_{0}^{2} \int_{\mathbb{T}^{2}} \delta \hat{\alpha}\left(k, k^{\prime}\right) \frac{e^{2 \pi i\left(k+k^{\prime}\right) x}}{\omega(k) \omega\left(k^{\prime}\right)} d k d k^{\prime}, \\
r_{x}^{(2,2)} & =2\left\{\mathbb{E}\left[(\tilde{\omega} * \mathfrak{q})_{0}(\tilde{\omega} * \mathfrak{q})_{x}\right]\right\}^{2}=2 \mathcal{E}_{0}^{2} \delta_{x, 0} .
\end{aligned}
$$

Here $\delta \hat{\alpha}\left(k, k^{\prime}\right)$ is given by (13.5). Therefore,

$$
\begin{aligned}
& \epsilon \mathbb{E}\left[\sum_{x} \phi_{x} J(\epsilon x)\right]^{2}=\epsilon \sum_{x, x^{\prime}} J(\epsilon x) J\left(\epsilon x^{\prime}\right) r_{x-x^{\prime}} \\
& =\frac{\epsilon \mathcal{E}_{0}^{2}}{2} \sum_{x, x^{\prime}} \int_{\mathbb{R}^{2} \times \mathbb{T}^{2}} e^{2 \pi i\left(k+k^{\prime}\right)\left(x-x^{\prime}\right)} e^{2 \epsilon \pi i\left(x p+x^{\prime} p^{\prime}\right)} \hat{J}(p) \hat{J}\left(p^{\prime}\right) F\left(k, k^{\prime}\right) d p d p^{\prime} d k d k^{\prime},
\end{aligned}
$$


with

$$
F\left(k, k^{\prime}\right):=\frac{\left[\delta \hat{\alpha}\left(k, k^{\prime}\right)+2 \omega(k) \omega\left(k^{\prime}\right)\right]^{2}}{\hat{\alpha}(k) \hat{\alpha}\left(k^{\prime}\right)} .
$$

Observe that $F(-k, k)=0$. Summing first over $x$ and then over $x^{\prime}$ we obtain that the utmost right hand side of (13.15) equals

$$
\sum_{n \in \mathbb{Z}} \int_{\mathbb{R}} \hat{J}(p) \hat{J}\left(\frac{n}{\epsilon}-p\right)\left(\int_{\mathbb{T}} F(-k-\epsilon p, k) d k\right) d p .
$$

Therefore (13.11) (thus also the conclusion of the proposition) is a consequence of the Lebesgue dominated convergence theorem and Lemma 13.1.

13.2.2. The pinned case. Then,

$$
\mathfrak{q}_{x}=\sqrt{\mathcal{E}_{0}} \int_{\mathbb{T}} \frac{e^{2 \pi i k x}}{\omega(k)} \hat{w}(d k) .
$$

We have $\mathbb{E} \phi_{x}=0$ with

$$
\begin{aligned}
& \mathbb{E} \phi_{x}^{(1)}=\mathcal{E}_{0} \int_{\mathbb{T}} \frac{\hat{\alpha}(0)-\hat{\alpha}(k)}{\hat{\alpha}(k)} d k, \\
& \mathbb{E} \phi_{x}^{(2)}=\mathcal{E}_{0}, \quad \mathbb{E} \phi_{x}^{(3)}=-\mathcal{E}_{0} \int_{\mathbb{T}} \frac{\hat{\alpha}(0)}{\hat{\alpha}(k)} d k .
\end{aligned}
$$

We let

$$
r_{x}:=\mathbb{E}\left(\phi_{x} \phi_{0}\right)=\sum_{i, i^{\prime}=1}^{3} r_{x}^{\left(i, i^{\prime}\right)},
$$

where $r_{x}^{\left(i, i^{\prime}\right)}:=\mathbb{E}\left(\tilde{\phi}_{x}^{(i)} \tilde{\phi}_{0}^{\left(i^{\prime}\right)}\right)$ and $\tilde{\phi}_{x}^{(i)}:=\phi_{x}^{(i)}-\mathbb{E} \phi_{x}^{(i)}, i=1,2,3$. We have

$$
\begin{aligned}
r_{x}^{(1,1)} & =\frac{1}{2} \sum_{x^{\prime}, x^{\prime \prime}} \alpha_{x-x^{\prime}} \alpha_{x^{\prime \prime}}\left\{\mathbb{E}\left[\left(\mathfrak{q}_{x}-\mathfrak{q}_{x^{\prime}}\right)\left(\mathfrak{q}_{x^{\prime \prime}}-\mathfrak{q}_{0}\right)\right]\right\}^{2} \\
& =\frac{\mathcal{E}_{0}^{2}}{2} \int_{\mathbb{T}^{2}}\left[\delta \hat{\alpha}\left(k, k^{\prime}\right)\right]^{2} \frac{e^{2 \pi i\left(k+k^{\prime}\right) x}}{\hat{\alpha}(k) \hat{\alpha}\left(k^{\prime}\right)} d k d k^{\prime},
\end{aligned}
$$

where $\delta \hat{\alpha}\left(k, k^{\prime}\right):=\hat{\alpha}(0)+\hat{\alpha}\left(k+k^{\prime}\right)-\hat{\alpha}(k)-\hat{\alpha}\left(k^{\prime}\right)$ and

$$
\begin{aligned}
r_{x}^{(2,1)} & =r_{x}^{(1,2)}=\sum_{x^{\prime}} \alpha_{x-x^{\prime}}\left\{\mathbb{E}\left[\left(\mathfrak{q}_{x}-\mathfrak{q}_{x^{\prime}}\right)(\tilde{\omega} * \mathfrak{q})_{0}\right]\right\}^{2} \\
& =\mathcal{E}_{0}^{2} \int_{\mathbb{T}^{2}} \delta \hat{\alpha}\left(k, k^{\prime}\right) \frac{e^{2 \pi i\left(k+k^{\prime}\right) x}}{\omega(k) \omega\left(k^{\prime}\right)} d k d k^{\prime}, \\
r_{x}^{(2,2)} & =2\left\{\mathbb{E}\left[(\tilde{\omega} * \mathfrak{q})_{0}(\tilde{\omega} * \mathfrak{q})_{x}\right]\right\}^{2}=2 \mathcal{E}_{0}^{2} \delta_{x, 0}, \\
r_{x}^{(3,1)} & =r_{x}^{(1,3)}=-\sum_{x^{\prime}} \alpha_{x-x^{\prime}} \hat{\alpha}(0)\left\{\mathbb{E}\left[\left(\mathfrak{q}_{x}-\mathfrak{q}_{x^{\prime}}\right) \mathfrak{q}_{0}\right]\right\}^{2} \\
& =-\mathcal{E}_{0}^{2} \int_{\mathbb{T}^{2}} \hat{\alpha}(0) \delta \hat{\alpha}\left(k, k^{\prime}\right) \frac{e^{2 \pi i\left(k+k^{\prime}\right) x}}{\hat{\alpha}(k) \hat{\alpha}\left(k^{\prime}\right)} d k d k^{\prime},
\end{aligned}
$$




$$
\begin{aligned}
r_{x}^{(3,2)}=r_{x}^{(2,3)} & =-2 \hat{\alpha}(0)\left\{\mathbb{E}\left[(\tilde{\omega} * \mathfrak{q})_{x} \mathfrak{q}_{0}\right]\right\}^{2} \\
& =-2 \mathcal{E}_{0}^{2} \int_{\mathbb{T}^{2}} \hat{\alpha}(0) \frac{e^{2 \pi i\left(k+k^{\prime}\right) x}}{\omega(k) \omega\left(k^{\prime}\right)} d k d k^{\prime}
\end{aligned}
$$

and

$$
r_{x}^{(3,3)}=2 \hat{\alpha}^{2}(0)\left\{\mathbb{E}\left[\mathfrak{q}_{x} \mathfrak{q}_{0}\right]\right\}^{2}=2 \mathcal{E}_{0}^{2} \int_{\mathbb{T}^{2}} \hat{\alpha}^{2}(0) \frac{e^{2 \pi i\left(k+k^{\prime}\right) x}}{\hat{\alpha}(k) \hat{\alpha}\left(k^{\prime}\right)} d k d k^{\prime}
$$

Therefore, we can write (13.15) with

$$
F\left(k, k^{\prime}\right):=\frac{1}{\hat{\alpha}(k) \hat{\alpha}\left(k^{\prime}\right)}\left\{-2 \hat{\alpha}(0)+2 \omega(k) \omega\left(k^{\prime}\right)+\delta \hat{\alpha}\left(k, k^{\prime}\right)\right\}^{2} .
$$

We have $F(-k, k)=0$. Repeating the argument made in the unpinned case, this time easier since we do not have to bother about possible singularities of $F(-k-\epsilon p, k)$ in the vicinity of 0 we conclude the assertion of the proposition for pinned chains.

\section{Auxiliary Results}

14.1. Some computations concerning the scattering kernel. Directly from (5.5) it follows that

$$
r\left(-k,-k^{\prime}\right)=-r\left(k, k^{\prime}\right)
$$

and

$$
\begin{aligned}
r & \left(k-\frac{p}{2}, k-k^{\prime}\right) r\left(k+\frac{p}{2}, k-k^{\prime}\right) \\
& =16\left[\mathfrak{s}^{2}(k)-\mathfrak{s}^{2}\left(\frac{p}{2}\right)\right] \mathfrak{s}^{2}\left(k^{\prime}\right)\left[\mathfrak{s}^{2}\left(k+k^{\prime}\right)-\mathfrak{s}^{2}\left(\frac{p}{2}\right)\right] .
\end{aligned}
$$

From (8.3) and (14.1) we have

$$
R\left( \pm k, \pm k^{\prime}, \pm p\right)=R\left(k, k^{\prime}, p\right) .
$$

Equality (14.2) allows us to write the following expansion

$$
R\left(k, k^{\prime}, p\right)=R\left(k, k^{\prime}\right)-\mathfrak{s}^{2}\left(\frac{p}{2}\right) R_{1}\left(k, k^{\prime}\right)+\mathfrak{s}^{4}\left(\frac{p}{2}\right) R_{2}\left(k, k^{\prime}\right) .
$$

Here $\mathfrak{s}(p), R\left(k, k^{\prime}\right)$ are $R_{1}\left(k, k^{\prime}\right)$ are given by (2.9), (5.13) and (9.7), respectively, and $R_{2}\left(k, k^{\prime}\right)=8 \mathfrak{f}_{+}\left(k^{\prime}\right)$ (see (9.8)). Using (2.7) we conclude

$$
R^{\prime}(k)=2 \pi(\mathfrak{s}(2 k)+\mathfrak{s}(4 k))
$$

and

$$
R^{\prime \prime}(k)=4 \pi^{2}\left(4 \mathfrak{c}^{2}(2 k)+\mathfrak{c}(2 k)-2\right) .
$$

Recall that $R_{\epsilon}(k)$ is given by (9.3). Since $R^{\prime \prime}(0)=12 \pi^{2}>0$, see (14.6), we conclude that for any $M>0$ one can find $\epsilon_{0}>0$, for which

$$
R_{\epsilon}(k) \approx R(k)+(\epsilon p)^{2}, \quad \forall k \in \mathbb{T}, \epsilon \in\left(0, \epsilon_{0}\right),|p| \leq M .
$$

Lemma 14.1. If $\omega(k) \approx|\sin (\pi k)|$ then, for any $M>0$ one can find $\epsilon_{0}>0$, for which

$$
\bar{\omega}(k, \epsilon p) \approx R_{\epsilon}^{1 / 2}(k), \quad k \in \mathbb{T}, \epsilon \in\left(0, \epsilon_{0}\right),|p| \leq M .
$$

If on the other hand $\omega(0)>0$ we have

$$
\left|\delta_{\epsilon} \omega(k, p)\right| \preceq R_{\epsilon}^{1 / 2}(k), \quad k \in \mathbb{T}, \epsilon \in\left(0, \epsilon_{0}\right),|p| \leq M .
$$


Proof of (14.8). Using (2.4) we obtain that

$$
\bar{\omega}(k, \epsilon p) \approx\left[\left|\sin \left(\pi\left(k-\frac{\epsilon p}{2}\right)\right)\right|+\left|\sin \left(\pi\left(k+\frac{\epsilon p}{2}\right)\right)\right|\right]
$$

for any $k \in \mathbb{T}, \epsilon \in(0,1], p \in \mathbb{R}$. Hence, for any $M>0$ one can find $\epsilon_{0}>0$, for which

$$
\bar{\omega}(k, \epsilon p) \approx|\sin (\pi k)|+\epsilon|p|, \quad \forall k \in \mathbb{T}, \epsilon \in\left(0, \epsilon_{0}\right),|p| \leq M .
$$

Estimate (14.8) follows from (2.7) and (14.7).

Proof of (14.9). Note that in case $\omega(0)>0$ we have $\omega \in C^{2}(\mathbb{T})$. Since $\omega(k)$ is even we have $\omega^{\prime}(0)=0$, therefore

$$
\left|\omega^{\prime}(k)\right| \preceq|\sin (\pi k)|, \quad k \in \mathbb{T} .
$$

Assume that $p \geq 0$. The case $p<0$ can be handled in a similar fashion. We can write

$$
\begin{aligned}
\delta_{\epsilon} \omega(k, p)-\omega^{\prime}(k) p= & \frac{1}{\epsilon} \int_{-\epsilon p / 2}^{\epsilon p / 2}\left[\omega^{\prime}(k+h)-\omega^{\prime}(k)\right] d h \\
= & \frac{1}{\epsilon} \int_{0}^{\epsilon p / 2}\left[\omega^{\prime}(k+h)-\omega^{\prime}(k)\right] d h \\
& +\frac{1}{\epsilon} \int_{0}^{\epsilon p / 2}\left[\omega^{\prime}(k-h)-\omega^{\prime}(k)\right] d h .
\end{aligned}
$$

The absolute value of the right hand side of (14.12) equals

$$
\begin{aligned}
& \frac{1}{\epsilon}\left|\int_{0}^{\epsilon p / 2} d h \int_{0}^{h}\left[\omega^{\prime \prime}\left(k+h_{1}\right)-\omega^{\prime \prime}\left(k-h_{1}\right)\right] d h_{1}\right| \\
& \quad \leq \frac{p^{2} \epsilon}{4} \sup _{\left|h_{1}\right| \leq \epsilon p / 2}\left|\omega^{\prime \prime}\left(k+h_{1}\right)-\omega^{\prime \prime}\left(k-h_{1}\right)\right| .
\end{aligned}
$$

From (14.11)-(14.13) it follows that

$$
\left|\delta_{\epsilon} \omega(k, p)\right| \preceq p(|\sin (\pi k)|+\epsilon p), \quad k \in \mathbb{T}, \epsilon>0, p \geq 0 .
$$

Combining this with (14.7) we conclude (14.9).

14.2. Proof of Proposition 10.1. From the third equation of (10.5)

$$
\bar{u}_{\epsilon,-}=\left(\lambda+\frac{2 \gamma}{\epsilon^{\delta}} R_{\epsilon}\right)^{-1}\left\{\widehat{U}_{\epsilon,-}(0)-\frac{2 \bar{\omega}}{\epsilon^{\delta}} \bar{u}_{\epsilon,+}-\frac{i \gamma R^{\prime} p}{2 \epsilon^{\delta-1}}\left(\bar{w}_{\epsilon,-}-\bar{w}_{\epsilon}\right)+\epsilon^{3-\delta} \bar{r}_{\epsilon}^{(3)}\right\} .
$$

Therefore

$$
\begin{aligned}
& \int_{\mathbb{T}} R_{\epsilon}\left[\bar{\omega}\left(\lambda \epsilon^{\delta}+\gamma R_{\epsilon}\right)^{-1}\right]^{2}\left|\bar{u}_{\epsilon,+}\right|^{2} d k \preceq \int_{\mathbb{T}} R_{\epsilon}\left|\bar{u}_{\epsilon,-}\right|^{2} d k \\
& +\int_{\mathbb{T}} R_{\epsilon}\left[\epsilon^{\delta}\left(\lambda \epsilon^{\delta}+\gamma R_{\epsilon}\right)^{-1}\right]^{2}\left|\widehat{U}_{\epsilon,-}(0)\right|^{2} d k \\
& +\int_{\mathbb{T}} R_{\epsilon}\left[\gamma \epsilon R^{\prime}\left(\lambda \epsilon^{\delta}+\gamma R_{\epsilon}\right)^{-1}\right]^{2}\left|\bar{w}_{\epsilon}\right|^{2} d k \\
& +\int_{\mathbb{T}} R_{\epsilon}\left[\epsilon^{3}\left(\lambda \epsilon^{\delta}+\gamma R_{\epsilon}\right)^{-1}\right]^{2}\left|\bar{r}_{\epsilon}^{(3)}\right|^{2} d k .
\end{aligned}
$$


Denote the terms appearing on the right hand side by $J_{j}, j=1,2,3,4$. Thanks to (10.7) we have $J_{1} \preceq \epsilon^{\delta-s}$. Also, ( since $\lambda \geq \lambda_{0}$ )

$$
R_{\epsilon}\left[\epsilon^{\delta}\left(\lambda \epsilon^{\delta}+\gamma R_{\epsilon}\right)^{-1}\right]^{2} \preceq R_{\epsilon} \epsilon^{2 \delta} \epsilon^{-\delta} \gamma^{-1} R_{\epsilon}^{-1} \preceq \epsilon^{\delta-s}
$$

and $\left(\right.$ since $\left(R^{\prime}\right)^{2} \preceq R$ ) we have

$$
R_{\epsilon}\left[\gamma \epsilon R^{\prime}\left(\lambda \epsilon^{\delta}+\gamma R_{\epsilon}\right)^{-1}\right]^{2} \preceq R_{\epsilon}^{2} \gamma^{2} \epsilon^{2} \gamma^{-2} R_{\epsilon}^{-2} \preceq \epsilon^{2} .
$$

From here we get that $J_{j} \preceq \epsilon^{\delta-s}, j=2,3$. Finally,

$$
R_{\epsilon}\left[\epsilon^{3}\left(\lambda \epsilon^{\delta}+\gamma R_{\epsilon}\right)^{-1}\right]^{2} \leq R_{\epsilon} \epsilon^{6} \epsilon^{-\delta} \gamma^{-1} R_{\epsilon}^{-1} \preceq \epsilon^{6-\delta-s},
$$

which also yields $J_{4} \preceq \epsilon^{\delta-s}$ that finally leads to an estimate

$$
\int_{\mathbb{T}} R_{\epsilon}\left[\bar{\omega}\left(\lambda \epsilon^{\delta}+\gamma R_{\epsilon}\right)^{-1}\right]^{2}\left|\bar{u}_{\epsilon,+}\right|^{2} d k \preceq \epsilon^{\delta-s} .
$$

To obtain the estimate of $\mathcal{D}\left(\bar{w}_{\epsilon}(\lambda, p)\right)$ it suffices to prove that $\mathcal{D}\left(\bar{u}_{\epsilon,+}(\lambda, p)\right) \preceq \epsilon^{\delta-s}$, which follows, provided we can show that

$$
\int_{\mathbb{T}} R(k)\left|\bar{u}_{\epsilon,+}(\lambda, p, k)\right|^{2} d k \preceq \epsilon^{\delta-s} .
$$

Divide integration in (14.18) into two regions: $\left[|k| \leq \epsilon^{(\delta-s) / 2}\right]$ and $\left[|k| \geq \epsilon^{(\delta-s) / 2}\right]$. In the first region we use $R(k) \preceq \epsilon^{\delta-s}$ and the bound on the $L^{2}(\mathbb{T})$ norm of $\overline{\bar{u}}_{\epsilon,+}(\lambda, p)$, see (10.4). Since, in the second region, $R_{\epsilon}\left[\bar{\omega}\left(\lambda \epsilon^{\delta}+\gamma R_{\epsilon}\right)^{-1}\right]^{2}$ is bounded from below by $\gamma^{-2}$, in the unpinned case ( $\mathrm{cf}(14.8)$ ) and by $\gamma^{-2} R_{\epsilon}^{-1}$ in the pinned one we can bound the integral over the region by $\epsilon^{\delta-s}$, due to (14.17). Hence, (14.18) follows.

Acknowledgements. The authors would like to express their gratitude to an anonymous referee for a careful reading of the manuscript and remarks that lead to the improvement of the manuscript.

Open Access This article is distributed under the terms of the Creative Commons Attribution 4.0 International License (http://creativecommons.org/licenses/by/4.0/), which permits unrestricted use, distribution, and reproduction in any medium, provided you give appropriate credit to the original author(s) and the source, provide a link to the Creative Commons license, and indicate if changes were made.

\section{References}

1. Basile, G., Bernardin, C., Olla, S.: A momentum conserving model with anomalous thermal conductivity in low dimension. Phys. Rev. Lett. 96, 204303 (2006). doi:10.1103/PhysRevLett.96.204303

2. Basile, G., Bernardin, C., Olla, S.: Thermal conductivity for a momentum conservative model. Commun. Math. Phys. 287, 67-98 (2009)

3. Basile, G., Bovier, A.: Convergence of a kinetic equation to a fractional diffusion equation. Markov Proc. Relat. Fields 16, 15-44 (2010)

4. Basile, G., Olla, S.: Energy diffusion in harmonic system with conservative noise. J. Stat. Phys. 155(6), 1126-1142 (2014). doi:10.1007/s10955-013-0908-4

5. Basile, G., Olla, S., Spohn, H.: Energy transport in stochastically perturbed lattice dynamics. Arch. Ration. Mech. 195(1), 171-203 (2009) 
6. Bernardin, C., Goncalves, P., Jara, M.: 3/4 Fractional superdiffusion of energy in a system of harmonic oscillators perturbed by a conservative noise. arXiv:1402.1562v1 (2014, preprint)

7. Bernardin, C., Olla, S.: Thermodynamics and non-equilibrium macroscopic dynamics of chains of anharmonic oscillators. Lecture Notes. https://www.ceremade.dauphine.fr/ olla/ (2014)

8. Braxmeier-Even, N., Olla, S.: Hydrodynamic limit for a Hamiltonian system with boundary conditions and conservative noise. Arch. Ration. Mech. Anal. 213, 561-585 (2014)

9. Da Prato, G., Zabczyk, J.: Stochastic Equations in Infinite Dimensions. Cambridge University Press, Cambridge (1992)

10. Komorowski, T., Jara, M., Olla, S.: A limit theorem for an additive functionals of Markov chains. Ann. Appl. Probab. 19(6), 2270-2300 (2009)

11. Komorowski, T., Olla, S.: Ballistic and superdiffusive scales in macroscopic evolution of a chain of oscillators. http://arxiv.org/pdf/1506.06465.pdf

12. Komorowski, T., Olla, S., Ryzhik, L.: Asymptotics of the solutions of the stochastic lattice wave equation. Arch. Rational Mech. Anal. 209, 455-494 (2013)

13. Komorowski, T., Stepien, L.: Long time, large scale limit of the Wigner transform for a system of linear oscillators in one dimension. J. Stat. Phys. 148, 1-37 (2012)

14. Lax, P.D.: Functional Analysis. Wiley, New York (2002)

15. Lepri, S., Livi, R., Politi, A.: Thermal conduction in classical low-dimensional lattices. Phys. Rep. 377, 1$80(2003)$

16. Lepri, S., Livi, R., Politi, A.: Heat conduction in chains of nonlinear oscillators. Phys. Rev. Lett. 78, 1896 (1997)

17. Lukkarinen, J., Spohn, H.: Kinetic limit for wave propagation in a random medium. Arch. Ration. Mech. Anal. 183(1), 93-162 (2006)

18. Mellet, A., Mischler, S., Mouhot, C.: Fractional diffusion limit for collisional kinetic equations. Arch. Ration. Mech. Anal. 199(2), 493-525 (2011)

19. Olla, S., Varadhan, S.R.S., Yau, H.T.: Hydrodynamic limit for a Hamiltonian system with weak noise. Commun. Math. Phys. 155, 523-560 (1993)

20. Spohn, H.: Nonlinear fluctuating hydrodynamics for anharmonic chains. J. Stat. Phys. 154(5), 11911227 (2014)

Communicated by H. Spohn 